\title{
Circuit refinement in mouse visual cortex during development
}

\author{
Dissertation \\ for the award of the degree \\ "Doctor rerum naturalium" \\ of the Georg-August-Universität Göttingen \\ within the IMPRS Neuroscience Program \\ of the Georg-August University School of Science (GAUSS) \\ submitted by \\ Man Ho Wong \\ from Hong Kong
}

Göttingen, 2017 


\section{Thesis Committee}

Asst. Prof. Dr. Dr. Oliver Schlüter, Department for Psychiatry and Psychotherapy, University Medical Center Göttingen; Department of Neuroscience, University of Pittsburgh (USA).

Prof. Dr. Siegrid Löwel, BFNT and School of Biology, University of Göttingen.

Prof. Dr. Tobias Moser, Institute for Auditory Neuroscience, University Medical Center Göttingen.

\section{Members of the Examination Board}

Referee: Asst. Prof. Dr. Dr. Oliver Schlüter, Department for Psychiatry and Psychotherapy, University Medical Center Göttingen; Department of Neuroscience, University of Pittsburgh (USA).

2nd Referee: Prof. Dr. Siegrid Löwel, BFNT and School of Biology, University of Göttingen.

3rd Referee: Prof. Dr. Tobias Moser, Institute for Auditory Neuroscience, University Medical Center Göttingen.

\section{Further members of the Examination Board}

Prof. Dr. Erwin Neher, Max Planck Institute for Biophysical Chemistry, Göttingen.

Dr. Jeong Seop Rhee, Max Planck Institute of Experimental Medicine, Göttingen.

Dr. Marion Silies, European Neuroscience Institute Göttingen.

Date of oral examination: $4^{\text {th }}$ August, 2017 


\section{Contents}

\begin{tabular}{ll}
\hline Abbreviations & 2
\end{tabular}

\begin{tabular}{ll}
\hline Abstract & 3
\end{tabular}

1 Introduction 5

1.1 Transmission of signals across synapses $\ldots \ldots \ldots \ldots \ldots$

1.1 .1 Structure of synapses $\ldots \ldots \ldots \ldots \ldots \ldots$

1.1 .2 Synaptic transmission $\ldots \ldots \ldots \ldots \ldots \ldots$

1.2 Synapses are the sites of regulation $\ldots \ldots \ldots \ldots \ldots \ldots$

1.2 .1 Regulating synaptic plasticity with AMPARs $\ldots \ldots \ldots$

$1.2 .2 \quad$ Silent synapses lack AMPAR-mediated transmission . . . . . 10

1.3 Synapse maturation during development . . . . . . . . . . . . . . 11

$\begin{array}{lll}1.3 .1 & \text { Silent synapses are the substrates of developmental plasticity } 12\end{array}$

1.3.2 Developing visual cortex as a model to study synapse matura-

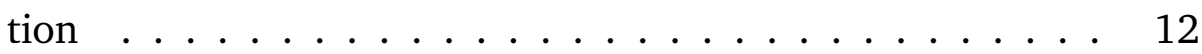

1.4 Scope of the study $\ldots \ldots \ldots \ldots \ldots \ldots$

$\begin{array}{lll}2 \text { Materials and Methods } & 17\end{array}$

2.1 Materials . . . . . . . . . . . . . . . . . . . . 17

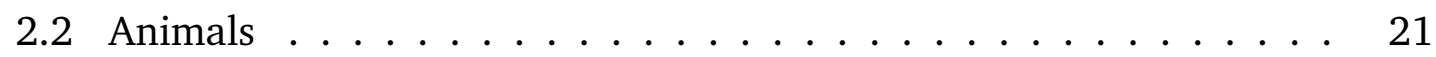

2.2 .1 Genetic background $\ldots \ldots \ldots \ldots \ldots \ldots \ldots . \ldots \ldots$

2.2 .2 Housing conditions and handling . . . . . . . . . . . 21

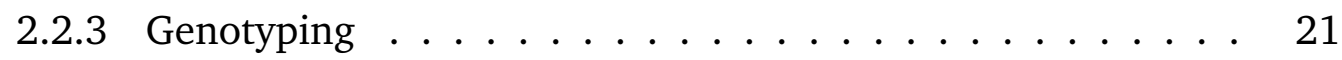

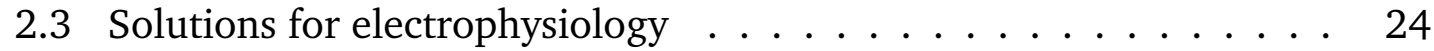

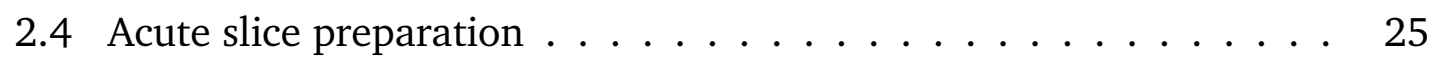

2.5 Electrophysiology . . . . . . . . . . . . . . . . . . . . 25

2.5 .1 The setup . . . . . . . . . . . . . . . . . . 25

2.5 .2 General recording configuration . . . . . . . . . . . . 27

2.5 .3 Evoked excitatory postsynaptic current (EPSC) . . . . . . . . 29

2.5 .4 Miniature EPSC (mEPSC) . . . . . . . . . . . . . . . . . . . . 29

2.5 .5 Paired-pulse ratio recording . . . . . . . . . . . . . . . . . 29

$2.5 .6 \quad$ NMDAR blocking by MK-801 $\ldots \ldots \ldots \ldots \ldots \ldots$ 
2.5 .7 AMPA response $\ldots \ldots \ldots \ldots \ldots$

2.6 Data analysis $\ldots \ldots \ldots \ldots \ldots \ldots \ldots \ldots$

2.6 .1 Evoked EPSC and AMPAR/NMDAR ratio . . . . . . . . 30

2.6 .2 mEPSC . . . . . . . . . . . . . . . . . . . . . . 31

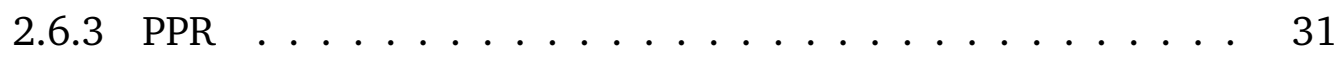

2.6 .4 NMDAR blocking by MK-801. . . . . . . . . . . . . . . 32

2.6 .5 AMPA-induced current . . . . . . . . . . . . . . . . 32

3 Results 33

3.1 Developmental changes in synaptic transmission and homeostasis of AMPAR-mEPSC frequency . . . . . . . . . . . . . . 33

3.1.1 AMPAR-mEPSC frequency increased upon eye opening but remained unchanged afterwards. . . . . . . . . . . . . . 34

$3.1 .2 \quad$ AMPAR/NMDAR ratio increased during development . . . . 36

3.2 Developmental change in presynaptic neurotransmitter release. . . 38

3.2.1 Paired-pulse ratio (PPR) of AMPAR-EPSC was unchanged. . 38

3.2 .2 Paired-pulse ratio (PPR) of NMDAR-EPSC increased . . . . . 39

3.2 .3 NMDAR blocking by MK-801 slowed down . . . . . . . . . . 41

3.3 Developmental change in labile synapses was not detected . . . . . 43

3.4 Novel role of AMPAR desensitization during development. . . . . . 47

3.4.1 AMPAR desensitization blocker TCM increased mEPSC frequency . . . . . . . . . . . . . . . . 47

3.4.2 TCM did not change the MK-801 blocking rate of NMDARs . $\quad 50$

3.4 .3 TCM only slightly increased evoked EPSC amplitude . . . . 50

3.5 Developmental increase in total surface AMPAR number . . . . . 52

$\begin{array}{lll}4 & \text { Discussion } & 57\end{array}$

4.1 circuit refinement parallel to silent synapse maturation . . . . . . 57

4.1.1 Eye opening (P13): critical point in synapse development . 57

4.1.2 Homeostasis of AMPAR-mEPSC frequency after eye opening 59

4.2 Developmental decrease in release probability . . . . . . . . . 62

$4.2 .1 \quad$ Silent synapses had higher release probability . . . . . . . 62

4.2 .2 Decrease in release probability during synapse maturation . 64

4.3 Unlocking AMPAR transmission by TCM . . . . . . . . . . . 65

4.3 .1 TCM activated AMPAR-containing idle sites . . . . . . . 65

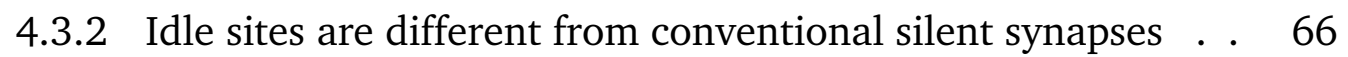

4.3 .3 Are idle sites silenced pre- or postsynaptically?. . . . . . . 67

4.4 Dissociation of spontaneous and evoked transmission at idle sites . 68

$4.4 .1 \quad$ Dissociation at presynapse or postsynapse? . . . . . . . . 69

4.4.2 Dissociation within same synapses or among different synapses? 70 
4.5 Revising PSD-95's role in AMPAR transmission . . . . . . . . . . . 71

4.5.1 $\quad$ PSD-95 is not a strict requirement for surface expression of

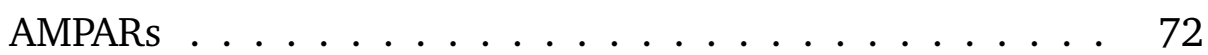

4.5 .2 Role of PSD-95 in silent synapse maturation . . . . . . . . 74

\begin{tabular}{ll}
\hline Appendix & 85
\end{tabular}

\begin{tabular}{lr}
\hline A Statistics & 87
\end{tabular}

\begin{tabular}{ll}
\hline B Supporting figures & 95
\end{tabular}

\begin{tabular}{ll}
\hline Acknowledgements & 99
\end{tabular}

\begin{tabular}{ll}
\hline Declaration & 101
\end{tabular}

\section{List of Figures}

$1.1 \quad$ Definition of silent synapses and AMPAR+ synapses in this study . . 11

$3.1 \quad$ Developmental changes in AMPAR-mEPSC frequency and amplitude of L2/3 pyramidal neurons of V1 . . . . . . . . . . . . . . . . 35

3.2 AMPAR/NMDAR ratio in layer 2/3 pyramidal neurons of V1 increased from P11 to P30 . . . . . . . . . . . . . . . . . . . 37

3.3 Paired-pulse ratio (PPR) of AMPAR+ synapses with 50 or $100 \mathrm{~ms}$ stimulus interval was unchanged during development. . . . . . . . . 40

$3.4 \quad$ Paired-pulse ratio (PPR) of AMPAR+ synapses was unchanged but PPR of NMDAR+ synapses increased during development . . . . . . . . . 41

3.5 MK-801 blocking rate of NMDARs was slower at P30 compared to P13 44

$3.6 \quad$ No difference was observed in AMPA-induced silencing of AMPAR+ synapses at different ages $\ldots \ldots \ldots 46$

3.7 AMPAR desensitization blocker TCM increased mEPSC frequency in WT at P13, P30 and in PSD-95 KO at P30 . . . . . . . . . . . 49 
3.8 MK-801 blocking rate of NMDARs was not affected by desensitization blocker TCM . . . . . . . . . . . . . . . . . . 51

3.9 AMPAR desensitization blocker TCM only had a subtle effect on evoked EPSC amplitude in wild-type mice at P30 . . . . . . . . . . . . 53

3.10 Developmental increase in AMPA response was observed in wild-type and PSD-95 KO mice . . . . . . . . . . . . . . . . 55

B.1 Developmental change in silent synapse fraction from P11 to P30 in L2/3 pyramidal neurons of V1 . . . . . . . . . . . . . . . 95

B.2 Developmental increase in spine density in L2/3 pyramidal neurons of

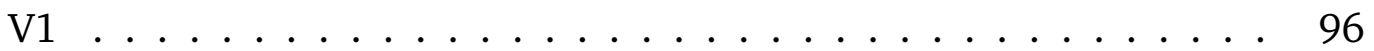

B.3 Desensitization blocker TCM had stronger effect on AMPAR-mEPSC frequency at P31 than at P16 . . . . . . . . . . . 97

\section{List of Tables}

$2.1 \quad$ List of chemicals, reagents and drugs $\ldots \ldots \ldots \ldots$

$2.2 \quad$ List of devices . . . . . . . . . . . . . . . . . . . 18

2.3 List of other materials $\ldots \ldots \ldots$. . . . . . . . . . . . . . . . 19

2.4 List of softwares . . . . . . . . . . . . . . . . . . 20

2.5 List of primers for genotyping . . . . . . . . . . . . . . . . . . . . . . . . . . . . 23

2.6 PCR programs . . . . . . . . . . . . . . . . 23

2.7 Expected sizes of gel bands . . . . . . . . . . . . . 23

A.1 Result summary . . . . . . . . . . . . . . . . 87

A.2 $\quad$ Statistical test results . . . . . . . . . . . . . . . 90 


\section{Abbreviations}

$\tau_{\text {fast }}$ time constant of fast component

$\tau_{\text {slow }}$ time constant of slow component

ACSF artificial cerebrospinal fluid

AMPA $\alpha$-amino-3-hydroxy-5-methyl-4-isoxazolepropionic acid

AMPAR $\alpha$-amino-3-hydroxy-5-methyl-4-isoxazolepropionic acid receptor

AMPAR+ synapse AMPAR-positive synapse

AMPAR-EPSC AMPAR-mediated EPSC

AMPAR-mEPSC AMPAR-mediated mEPSC

AMPAR-PPR paired-pulse ratio of AMPAR-EPSCs

AMPAR/NMDAR ratio ratio of AMPAR-EPSC to NMDAR-EPSC

EPSC excitatory postsynaptic current

EPSP excitatory postsynaptic potential

KO knock-out

LTP long-term synaptic potentiation

mEPSC miniature excitatory postsynaptic current 
MK-801 use-dependent NMDAR blocker: blocks only the activated NMDARs

NBQX 2,3-dihydroxy-6-nitro-7-sulfamoyl-benzo[f] quinoxaline-2,3-dione

NMDAR N-methyl-D-aspartate receptor

NMDAR+ synapse NMDAR-positive synapse

NMDAR-EPSC NMDAR-mediated EPSC

NMDAR-PPR paired-pulse ratio of NMDAR-EPSCs

ODP ocular dominance plasticity

PCR polymerase Chain Reaction

Percent $_{\text {fast }}$ percentage of fast component

PPR paired-pulse ratio

PSD post-synaptic density

PSD-95 post-synaptic density protein 95

TCM trichlormethiazide

TTX tetrodotoxin

V1 primary visual cortex

WT wild-type 


\section{Abstract}

Circuit refinement is an important developmental process ensuring correct wiring of synaptic connections. During circuit refinement, favorable connections are generated or strengthened while unfavored ones are weakened or eliminated. Such rewiring of synaptic connections during development is not a random but a sophisticated process with activity or experience-dependent plasticity involved. $\alpha$-amino-3-hydroxy-5-methyl-4-isoxazolepropionic acid receptor (AMPAR)-silent synapses, lacking AMPARs and therefore not transmitting at resting potential, act as the substrates for plasticity and likely play an essential role in circuit refinement.

Large amounts of silent synapses at early stages of development form a malleable network of synapses, potentially providing a template for circuit refinement. Huang et al. (2015) show that silent synapse maturation determines the closure of a critical period in an experience-dependent manner. Specifically, they observed a developmental decrease in the silent synapse fraction during the critical period. This suggests that synapse maturation is involved in the circuit refinement during this period, functioning as a mechanism for the formation of favorable connections. Nevertheless, the silent synapse fraction is only a relative measurement which does not tell us about the changes of the exact number of silent or AMPAR-positive $($ AMPAR + ) synapses (synapses conducting AMPAR-mediated transmission). To understand the role of synapse maturation in circuit refinement, this study began with identifying key changes in synapse connectivity during this process, specifically the silent synapse and AMPAR+ synapse numbers.

With miniature excitatory postsynaptic current (mEPSC) recording, I found that there was a two-fold increase in mEPSC frequency and a significant decrease in mEPSC amplitude upon eye opening, but both remained unchanged afterwards. The unchanged frequency after eye opening suggests that AMPAR+ synapse number remained unchanged after eye opening, in contrast to the decrease in silent synapse fraction during the same period. Taken together, these results indicated a 
homeostasis of mEPSC frequency during circuit refinement. This homeostasis could be achieved through certain compensatory mechanisms which counteract the effect of silent synapse maturation on mEPSC frequency.

In this study, I assessed three potential mechanisms for the homeostasis of mEPSC frequency: changes in release probability, maturation of labile synapses (synapses that can be silenced by repeated activation) and AMPAR desensitization during development. While changes in release probability and labile synapse maturation were not detected, I found that mEPSC frequency increased by more than three fold by blocking desensitization with trichlormethiazide (TCM), but a similar change in evoked EPSC amplitude was absent. Moreover, release probability assessed by NMDAR blockage with MK-801 was unaffected by TCM. These results not only provide evidence that desensitization of a certain type of AMPAR + synaptic transmission sites may account for the homeostasis, these transmission sites (referred as "idle sites" in this study) may also explain the dissociation of evoked and spontaneous neurotransmitter release observed in other studies.

To conclude, the developmental homeostasis of mEPSC frequency reported in this study suggests a physiological role of spontaneous transmission in synaptic plasticity. It provides an additional mechanism for homeostatic plasticity, distinct from the current view of synaptic scaling. Furthermore, this study proposed an unknown class of transmission sites containing desensitized AMPARs, the "idle sites". In addition to potentially mediating the homeostasis of mEPSC frequency, idle sites also serve as a direct evidence for the dissociation of spontaneous and evoked transmission. With these findings, this study open new directions in the study of synaptic transmission. 


\section{Introduction}

Our brain connects us to the external world. However, it is not ready-made at birth: it matures during development with increasing power. Central to this process is the maturation of a tiny structure called "synapse". Synapses connect neurons and they are the sites of regulation in neural activity. During brain development, synapse maturation takes place and synaptic connections are refined. The expected outcome of this refinement would be an optimized neural network with increased efficiency. However, it remains unclear what exact changes happen in synapse connectivity, and how they happen during development. This study addresses these questions by using visual cortex as a model of synaptic refinement during development. In this chapter, I will start with introducing the signal transmission across synapses, followed by highlighting the central role of synapses in the regulation of neural plasticity. Finally, I will discuss the importance of synapse maturation during development which leads to the main question of this study: what role does synapse maturation play in developmental circuit refinement?

\subsection{Transmission of signals across synapses}

The most unique feature of neurons that distinguishes them from other body cells is their capability to communicate with each other through synapses. A synapse is the interface between two neurons across where signals are transmitted. It is typically formed between an axon of the presynaptic neuron and a dendrite of the postsynaptic neuron. In the central nervous system, a class of molecules called neurotransmitters are used for passing information between the presynaptic and postsynaptic neurons. During synaptic transmission, neurotransmitter is released from the presynaptic terminal of an axon and diffuses across the synaptic cleft to bind the receptors on the postsynaptic membrane. A synapse can be excitatory or inhibitory, depending on whether the signal transmitted across it increases or decreases the likelihood of signal firing in the postsynaptic neuron. Pyramidal neurons (the principal neurons in the cortex) form excitatory synapses onto their postsynaptic targets, while interneurons form inhibitory synapses. In the brain, excitatory synapses typically use glutamate as the neurotransmitter while inhibitory synapses usually use gamma-Aminobutyric acid (GABA). Because of the dominant role that pyramidal neurons play in neural activities in the brain, they are the 
targets of the investigation in this study. The following discussion will focus on pyramidal neurons and their excitatory synapses.

\subsubsection{Structure of synapses}

\section{Presynaptic terminal}

Presynaptic terminal is a specialized structure for neurotransmitter release. Neurotransmitter molecules are synthesized and loaded into the synaptic vesicles which are later trafficked to the synapse. Synaptic vesicles can be found in as least two pools: the reserve pool away from the active zone where neurotransmitter is released, and the releasable pool within the active zone. Neurotransmitter molecules are released into the synaptic cleft through vesicle fusion at release sites on the presynaptic membrane. This process is mediated by SNAP (soluble NSF attachment protein) receptor protein (SNARE) complex formed between the vesicle and the presynaptic membrane.

\section{Postsynaptic spine}

In the postsynaptic spine, there is a protein dense region post-synaptic density (PSD), The PSD is opposite to the active zone of the presynaptic terminal, to where neurotransmitter receptors cluster. The major two classes of glutamate receptors are N-methyl-D-aspartate receptors (NMDARs) and $\alpha$-amino-3-hydroxy5-methyl-4-isoxazolepropionic acid receptors (AMPARs), These receptors interact with post-synaptic density protein 95 (PSD-95) either directly or indirectly in the PSD.

\subsubsection{Synaptic transmission}

\section{Transmission at a glance}

The arrival of an action potential at the presynaptic terminal triggers the influx of calcium ions and causes the release of neurotransmitter such as glutamate. Upon binding with glutamate, glutamate receptors such as AMPARs or NMDARs on the postsynaptic membrane open and allow ions flow into or out of the postsynaptic 
spine. Because of the electrochemical gradients across the postsynaptic membrane, opening of these receptors causes a net influx of cations which depolarizes the postsynaptic membrane (i.e. membrane potential becomes less negative than resting membrane potential), giving rise to excitatory postsynaptic potentials (EPSPs), EPSPs at the same neuron can be summed spatially (i.e. for EPSP arose at different synapses of the same neuron) or temporally (i.e. for EPSP arose at different time). When the EPSP reaches the threshold for firing of action potentials, an action potential will be fired and passed on to the postsynaptic neurons.

\section{Quantal nature of synaptic transmission}

As mentioned above, neurotransmitter molecules are packed into synaptic vesicles in presynaptic terminals. The amount of neurotransmitter packed in one vesicle, i.e. a quantum, is the minimal amount of neurotransmitter that a presynaptic terminal can release. The postsynaptic response to one quantum (i.e. quantal response, q) yields a miniature EPSP (mEPSP). An EPSP is composed of steps of mEPSP del Castillo and Katz, 1954). Therefore, in the simplest scenario, the size of an EPSP can be determined by:

$$
\mathrm{EPSP}=q \cdot n \cdot \operatorname{Pr}
$$

where $\mathrm{n}$ is the number of quanta and $\mathrm{Pr}$ is the release probability (the likelihood of vesicle fusion). Presumably, each release site in synapses of the central nervous system releases one quantum at a time (Frerking et al. 1997). Therefore, $\mathrm{n}$ can be used to estimate the number of independent release sites involved in one EPSP response. $\mathrm{q}$ is mainly determined by the amount of neurotransmitter in one quantum and the number of receptors on the postsynaptic membrane.

\section{Synaptic transmission and brain functions}

Apart from passing information, synaptic transmission play a central role in brain functions. A neuron can integrate the inputs from different pathways and generates outputs to other neurons. It acts as the basic computational unit of the brain, forming a network with other neurons for information processing and storage. The activity of a neuron can be regulated through changing the properties of its synapses. 


\subsection{Synapses are the sites of regulation}

Neural activities are constantly changing in response to experiences. One of the key features of the brain is the ability to alter its own properties to accomplish different tasks, i.e. plasticity. Plasticity plays a central role in regulating the neural activities, and therefore the brain functions such as learning and memory. It is also essential for the brain development. Changes in neural activity can be achieved by regulating the strength of the synapses, i.e. how likely and how strong a postsynaptic response will be to a presynaptic signal. It can be changed through the regulation of the machinery for synaptic transmission. Neural activity can also be regulated by changing the number of synapses involved in synaptic transmission through e.g. "switching off" the synapses by synapse silencing. As the pivot of synaptic transmission, synapses are the major sites for the regulation of neural activity.

\subsubsection{Regulating synaptic plasticity with AMPARs}

Synaptic plasticity allows changes in synaptic strength, such as potentiation or depression. There are different mechanisms for synaptic plasticity, relying on presynaptic and/or postsynaptic changes in synapse properties. Postsynaptically, synaptic strength can be altered by the surface expression of the major glutamate receptors involved in excitatory transmission, AMPARs. Trafficking of AMPARs underlies the regulation of synaptic strength.

\section{Synaptic strength involves AMPAR trafficking}

AMPARs can be found on the membrane surface or in intracellular regions. On the membrane, they are found on the synaptic or extrasynaptic membrane. Because of the low sensitivity to glutamate (Patneau and Mayer, 1990), only the postsynaptic AMPARs near or opposite to the presynaptic release site are transmitting. Two pathways of AMPAR trafficking to the postsynaptic site have been described (Chater and Goda, 2014. AMPARs can be first inserted into the extrasynaptic membrane (e.g. at the soma) by exocytosis, then incorporated into the postsynaptic site at the synapse through lateral diffusion Adesnik et al. 2005). Alternatively, AMPARs can be inserted directly into the synapse or near it through exocytosis Kennedy et al. 2010. 
AMPAR trafficking underlies the basic mechanism for long lasting changes in synaptic strength. One of the most studied forms of plasticity, long-term synaptic potentiation (LTP), involves strengthening of a synapse through increasing AMPAR numbers at the synapse. However, it is not completely understood which AMPAR trafficking pathways LTP employs to increase the synaptic AMPAR number. LTP has been reported to adopt either (Makino and Malinow, 2009, Yang et al., 2008) or both pathways (Patterson et al. 2010) for the upregulation of AMPAR numbers. Despite the ongoing debate on the pathways of AMPAR trafficking in the regulation of synaptic strength, the general consensus is that the postsynaptic form of synaptic plasticity is primarily mediated by the change in the number of synaptic AMPARs.

\section{Neural activity dictates AMPAR mobility and thus synaptic strength}

Synaptic strength is not solely regulated by the number of synaptic AMPARs, since AMPARs at the membrane surface can be highly mobile. AMAPRs inserted through either of the above trafficking pathways require stabilization at the synapse. AMPAR mobility provides another mechanism for a neuron to regulate its synaptic strength. It has been linked to synaptic plasticity. For example, the exchange of peri-synaptic and synaptic AMPARs allows rapid regulation of synaptic strength. It has been shown that when the mobility of surface AMPARs is reduced by e.g. crosslinking of the receptors, recovery from depression in synaptic strength due to AMPAR desensitization (resulted from prolonged receptor activation) is slowed down (Heine et al. 2008).

Synaptic strength can be regulated by altering the AMPAR mobility at the surface Groc et al. 2004). AMPAR mobility depends on several factors, such as the developmental stage and the AMPAR subunits. For instance, GluR2 (a subunit of AMPARs) has lower mobility in mature neurons (Borgdorff and Choquet, 2002). The level of neural activity has been shown to influence mobility as well. Mobility is slower with higher level of neural activity (Groc et al., 2004). Besides, neural activity also regulates the capturing of AMPARs. Chronic blockade of presynaptic release has been shown to impair the trapping of GluR1 subunits of AMPARs at the synapse (Ehlers et al. 2007). Neural activity regulates AMPAR mobility which in turn governs the synaptic strength. This enables neurons to control the synaptic transmission in response to the needs through monitoring activity. This activitydependent form of plasticity is particularly important during the development of sensory system such as vision. 


\subsubsection{Silent synapses lack AMPAR-mediated transmission}

As discussed above, regulation of AMPAR expression at synapses is the major mechanism for synaptic plasticity. It has been found that there are synapses which are completely devoid of AMPAR-mediated transmission, though these synapses still have NMDAR transmission at depolarized membrane potential (Isaac et al., 1995; Liao et al. 1995. These synapses are referred as the "silent synapses" due to the lack of transmission at resting potential. Silent synapses are the substrate of synaptic plasticity. During LTP, these synapses can be "unsilenced" as indicated by the emergence of AMPAR transmission (Bredt and Nicoll, 2003, Feldman et al., 1999). However, since there is a debate on how synapses are silenced (Kerchner and Nicoll, 2008), there is no concensus on the mechanism of LTP-induced unsilencing.

\section{Mechanisms of synapse silencing}

Two principle mechanisms of synapse silencing have been proposed with different supporting evidences. Synapses may be pre- or postsynaptically silent (in popular metaphors: synapses could be "mute" or "deaf"). The most intuitive mechanism is probably the postsynaptic one. Accordingly, synapses could be silenced due to the absence of functional AMPARs while glutamate release is still present Isaac et al. 1995, Liao et al., 1995). These synapses contain NMDARs, allowing them to be detected at a depolarized membrane potential when the $\mathrm{Mg}^{2+}$ block is removed (Nowak et al. 1984). Postsynaptically silent synapses have been observed experimentally by activating glutamate receptors locally with glutamate uncaging at individual spines (Beique et al. 2006, Busetto et al. 2008.

Presynaptic silencing has also been proposed by several studies (Balland et al. 2008, Choi et al. 2000, Gasparini et al., 2000, Kullmann et al., 1996). Presynaptically, synapse silencing is suggested to be caused by the impaired neurotransmitter release. This theory relies on the lower glutamate sensitivity of AMPARs compared to NMDARs (Patneau and Mayer, 1990). NMDARs can be activated at depolarized membrane potential by the low concentration of glutamate in the synaptic cleft which is not sufficient to activate AMPARs. There are two hypotheses accounting for the low glutamate concentration. The first purposed hypothesis suggests that while there could be no presynaptic release, there is a "spillover" of glutamate from neighboring synapses (Kullmann et al. 1996). Another hypothesis suggests that 

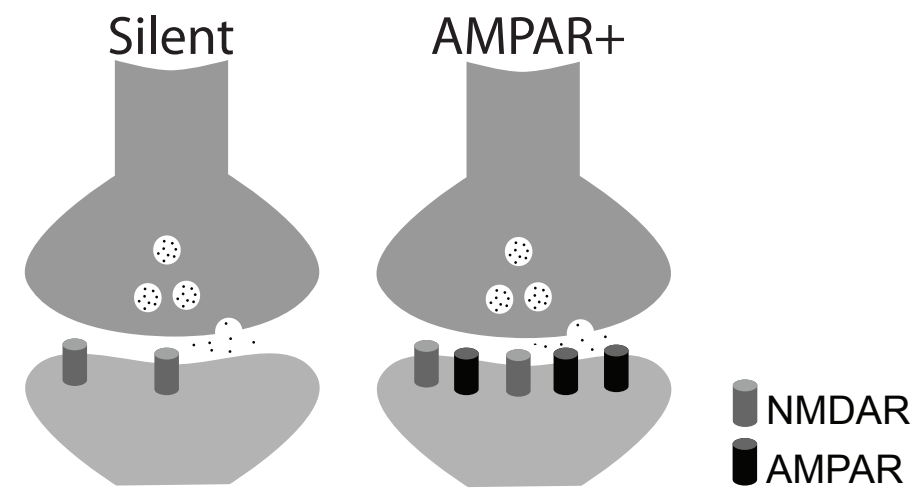

Fig. 1.1. Definition of silent synapses and AMPAR+ synapses in this study. In this study, synapses without AMPARs are referred as "silent synapses" while those with AMPARs are "AMPAR+ synapses".

there is presynaptic release with restricted fusion pore opening Choi et al. 2000, Gasparini et al. 2000) (i.e. "whispering" synapses).

Nevertheless, the pre- or postsynaptic mechanisms of synapse silencing are not mutually exclusive. Two types of silent synapses with opposite loci of silencing may exist. To avoid confusion, I will refer the transmitting synapses as AMPARpositive synapses (AMPAR+ synapses), and the silent synapses which do not contain AMPARs as simply "silent synapses", unless explicitly stated otherwise (figure1.1). NMDAR-positive synapses (NMDAR+ synapses) include both silent and AMPAR+ synapses.

\section{Silent synapses and plasticity}

Silent synapses have been proposed as the substrates for LTP. It is believed that expression of LTP leads to AMPAR trafficking to the postsynaptic sites in an activitydependent manner (Bredt and Nicoll, 2003, Derkach et al., 2007, Feldman et al., 1999). Since LTP has been suggested as the basic mechanisms for brain functions such as learning and memory, LTP-driven unsilencing suggests the important role that silent synapses play in brain functions.

\subsection{Synapse maturation during development}

As the primary sites for the regulation of neural activity, synapses undergo extensive modification during development. Among all different types of synapses, silent 
synapses have the largest potential for modifications because of the lack of AMPAR transmission. Maturation of silent synapses into functional AMPAR + synapses underlies not only the basis of synaptic plasticity, but it also plays an essential role during development. Despite the inability to transmit signals at resting potential, the abundance of silent synapses during earlier developmental stages suggests that silent synapses have functional importance during the development.

\subsubsection{Silent synapses are the substrates of developmental plasticity}

Cortical development involves experience-driven shifts in synaptic plasticity and connectivity Sur et al. 2013). By perturbing sensory experience early in development, change in synaptic plasticity at intracortical pathways can be observed Cheetham et al., 2007, Katz and Shatz, 1996, Takahashi et al., 2003). Besides, it has been shown that experience strengthens synaptic connections by promoting AMPAR trafficking to the postsynaptic membrane (Takahashi et al. 2003). Early silent synapses require experience-driven neural activity for unsilencing through insertion of AMPARs. Various brain regions have been shown to regulate developmental plasticity with synapse unsilencing in an experience-dependent manner,

including barrel cortex (Ashby and Isaac, 2011) and visual cotex (Huang et al. 2015). These findings suggest that synapse unsilencing is a general mechanism for developmental plasticity.

\subsubsection{Developing visual cortex as a model to study synapse maturation}

\section{Synapse maturation in visual cortex}

Visual cortex is an ideal model to study synaptic plasticity because of the ease of control of its major input, vision. Its complex functional architecture relating to diverse receptive fields of individual neurons has been appreciated since the groundbreaking study done by Hubel and Wiesel (1959). The development of the neural circuits leading to different receptive field properties has interested scientists for decades. Visual cortex is one of the most characterized cortical area for experience-dependent plasticity, with a substantial number of the studies focusing on binocular plasticity and ocular dominance plasticity (ODP). 
Development has influence on how visual information is precisely tuned and represented in primary visual cortex (Espinosa and Stryker, 2012). For example, tuning of a lot of sensory modalities, such as ODP, happens in early development during a time window referred as the "critical period" . Critical periods exist in the development of different brain functions. These periods of peak plasticity when many basic brain functions are set up by experiences. Plasticity of different sensory and cognitive functions are reduced or even lost after this time window. A daily life example would be our ability to learn our native languages easily during young age. In visual cortex, the critical period is around P21-35 for mice Espinosa and

Stryker, 2012, Gordon et al., 1996). ODP, a popular model plasticity used to study the experience-dependent plasticity during development, exists during its critical period.

ODP is the neuron's ability to change the preference to the activation by one of the eyes. Huang et al. (2015) found that silent synapse maturation is central to the duration of the critical period for ODP. The maturation of silent synapses closes the critical period, while abolishing it by knocking out PSD-95 leads to life-long ODP. Specifically, they found that the silent synapse fraction decreases progressively during development while the fraction is maintained at high level (around 50\%) in PSD-95 knock-out mice. This suggests that circuit refinement during critical period likely relies on the generation of functional synapses through silent synapse maturation. Besides, their results also underscore the key role that PSD-95 plays in synaptic maturation and refinement during development.

\section{Synapse maturation and refinement}

Indeed, neural circuits (e.g. in visual cortex: Hoy and Niell 2015); Hubel and Wiesel (1977)) for many sensory modalities are formed at birth or before receiving the corresponding patterned experience (in the case of visual cortex, this would be the time before eye opening). For example, the preference of a neuron to the activation by contralateral eye is present already before eye opening, well ahead of the critical period (Horton and Hocking, 1996). Besides, orientation selectivity (a neuron's preference to the activation by specific orientation of the visual input) is present before the critical period though the selectivity is different for the inputs from the two individual eyes. Instead of establishing neural circuits from birth, these circuits for different sensory modalities are "built-in" at birth and are only refined during critical periods in an experience-dependent manner (Hoy and Niell, 2015, Smith and Trachtenberg, 2007, Wang et al., 2010. 
Abundant synaptic connections exist during early development. While they might have specific physiological functions in early stages, it has also been suggested that they allow refinement of neural circuits by extrinsic experience or through the competition among themselves (Riccomagno and Kolodkin 2015). Refinement of synaptic connections during development ensures proper functioning of the brain. It can increase the accuracy of sensory input and fix the errors arising from wrong afferent projection during development.

Circuit refinement in sensory systems involves selective activation or elimination of functional connections relying on experience. The study done by Huang et al. 2015) reveals the strong association between experience-dependent silent synapse maturation and the critical period during which circuit refinement presumably occurs. This suggests that silent synapse maturation should play an important role in circuit refinement. Since experience-dependent synapse maturation happens not only in visual cortex but also in other cortical regions such as barrel cortex Ashby and Isaac, 2011, a network of vast number of silent synapses during early development likely acts as a template for the sculpting of neural network by circuit refinement.

\subsection{Scope of the study}

\section{The main question: what role does synapse maturation play in developmental circuit refinement?}

Synaptic refinement during development involves formation of favorable connections and elimination of the unfavored ones. Silent synapses provide a morphological template for this to happen. Huang et al. 2015) have shown that the silent synapse fraction decreases progressively from the time before eye opening to the adult stage after the critical period, hinting that there could be an overall change in AMPAR + synapse number due to silent synapse maturation during developmental circuit refinement. Nevertheless, a change in the silent synapse fraction does not necessarily associate with a change in the absolute number of synapses. Besides, synaptic pruning or synaptogenesis may happen in parallel of silent synapse maturation. In order to understand the role of synapse maturation in circuit refinement, we have to first identify the exact changes in synaptic connections during this process, specifically the change in silent or AMPAR+ synapse number. 


\section{Studying synapses through electrical properties}

Synaptic transmission with electrical signals is the primary function of a neuron. The most direct way to study the changes in synaptic connections would be to look at the changes in a neuron's electrical properties with electrophysiology. The advantage of electrophysiological study over biochemical or morphological study is that, it provides a direct readout of a neuron's activity despite the complexity of the regulation of synaptic transmission. Because of the complexity of biological system, morphological changes do not always reflect functional changes. There is often a dissociation between the two aspects. For example, silent spines have been described to have anatomical features of mature spines Ashby and Isaac, 2011. This problem can be avoided with electrophysiological study.

In this study, I studied the changes in synaptic connectivity through electrophysiological recording of the postsynaptic responses at AMPAR+ synapses. Specifically, I examined the synaptic strength by recording the excitatory postsynaptic current (EPSC), and the quantal response by recording miniature excitatory postsynaptic current (mEPSC) (the current that gives rise to mEPSP) of a principle excitatory neuron (i.e. the pyramidal neurons) in layer $2 / 3$ of the primary visual cortex (V1). The amplitude of an EPSC tells us how strong the synaptic input is, which is influenced by many factors including the number of AMPAR + synapses, number of postsynaptic AMPARs and release probability. The change in frequency and amplitude of mEPSC responses during development predicts the change in the number of synapses and the change in quantal size (which most likely reflects postsynaptic AMPAR number). With mEPSC recordings, I could identify whether there is a change in AMPAR+ synapse number and AMPAR expression level during circuit refinement. To study the mechanisms leading to those changes, I have also examined the change in presynaptic release (by recording paired-pulse ratio (PPR) and the NMDAR blocking rate of an use-dependent NMDAR blocker MK-801), activation-induced silencing of AMPAR + synapses (by recording mEPSCs with and without AMPAR agonist AMPA), AMPAR desensitization (by recording mEPSCs and EPSCs in the presence of an AMPAR desensitization blocker trichlormethiazide, TCM) and changes in the expression of total surface AMPARs during development (by recording EPSCs evoked by AMPA). Since PSD-95 KO mice are known to have a high silent synapse fraction maintained through out development Huang et al. 2015), they were used in this study as controls with high silent synapse fraction. By identifying the key synaptic changes due to synaptic refinement during development, this study provides new angles for future investigations. 



\section{Materials and Methods}

\subsection{Materials}

Table 2.1. List of chemicals, reagents and drugs

\begin{tabular}{|c|c|}
\hline Chemical/reagent/drug & Supplier \\
\hline PBND & Homemade \\
\hline Agarose & Invitrogen, Waltham, USA \\
\hline AMPA & Carl Roth, Karlsruhe, Germany \\
\hline Bromophenol blue & Carl Roth, Karlsruhe, Germany \\
\hline $\mathrm{CaCl}_{2} \cdot 2 \mathrm{H}_{2} \mathrm{O}$ & Carl Roth, Karlsruhe, Germany \\
\hline Cesium gluconate & Homemade \\
\hline Choline chloride & Sigma-Aldrich Chemie, Munich, Germany \\
\hline $\mathrm{CsCl}$ & Carl Roth, Karlsruhe, Germany \\
\hline $\mathrm{CsOH}$ & Sigma-Aldrich Chemie, Munich, Germany \\
\hline dNTP & Bioline, Luckenwalde, Germany \\
\hline EGTA & Carl Roth, Karlsruhe, Germany \\
\hline Ethidium bromide & Carl Roth, Karlsruhe, Germany \\
\hline Gelatine & Carl Roth, Karlsruhe, Germany \\
\hline Glucose & Sigma-Aldrich Chemie, Munich, Germany \\
\hline Glycerol & Carl Roth, Karlsruhe, Germany \\
\hline HEPES & Carl Roth, Karlsruhe, Germany \\
\hline Isoflurane & AbbVie, North Chicago, USA \\
\hline $\mathrm{KCl}$ & Carl Roth, Karlsruhe, Germany \\
\hline Kynurenic acid & Sigma-Aldrich Chemie, Munich, Germany \\
\hline Mango Taq Polymerase & Bioline, Luckenwalde, Germany \\
\hline $\mathrm{MeSO}_{3} \mathrm{H}$ & Fluka, Buchs, Switzerland \\
\hline MgATP & Sigma-Aldrich Chemie, Munich, Germany \\
\hline $\mathrm{MgCl}_{2} \cdot 6 \mathrm{H}_{2} \mathrm{O}$ & Sigma-Aldrich Chemie, Munich, Germany \\
\hline $\mathrm{MgSO}_{4} \cdot \mathrm{H}_{2} \mathrm{O}$ & Carl Roth, Karlsruhe, Germany \\
\hline MK-801 & HelloBio, Bristol, UK \\
\hline $\mathrm{Na}_{2} \mathrm{ATP}$ & Sigma-Aldrich Chemie, Munich, Germany \\
\hline $\mathrm{NaCl}$ & Carl Roth, Karlsruhe, Germany \\
\hline NaGTP & Sigma-Aldrich Chemie, Munich, Germany \\
\hline
\end{tabular}


Table 2.1. List of chemicals, reagents and drugs

\begin{tabular}{ll}
\hline Chemical/reagent/drug & Supplier \\
\hline $\mathrm{NaH}_{2} \mathrm{PO}_{4}$ & Sigma-Aldrich Chemie, Munich, Germany \\
$\mathrm{NaHCO}_{3}$ & Sigma-Aldrich Chemie, Munich, Germany \\
$\mathrm{NBQX}$ & Abcam, Cambridge, UK \\
Nonident P40 & Fluka, Buchs, Switzerland \\
Picrotoxin & Abcam, Cambridge, UK \\
Primers & MPI-EM, Göttingen, Germany \\
Proteinase K, 1:100 & Carl Roth, Karlsruhe, Germany \\
dilution & \\
QX314-Cl & Sigma-Aldrich Chemie, Munich, Germany \\
sodium ascorbate & Fluka, Buchs, Switzerland \\
Sodium pyruvate & Sigma-Aldrich Chemie, Munich, Germany \\
Sodium tetraborate & Sigma-Aldrich Chemie, Munich, Germany \\
Sucrose & Carl Roth, Karlsruhe, Germany \\
TEA-Cl & Fluka, Buchs, Switzerland \\
tetradotoxin (TTX) & HelloBio, Bristol, UK \\
TNK buffer (10X) & Homemade \\
Trichlormethiazide & Sigma-Aldrich Chemie, Munich, Germany \\
(TCM) & \\
Tris & Carl Roth, Karlsruhe, Germany \\
Tween20 & Carl Roth, Karlsruhe, Germany \\
\hline
\end{tabular}

Table 2.2. List of devices

\begin{tabular}{ll}
\hline Device & Supplier \\
\hline $\begin{array}{l}\text { Amplifier (MultiClamp } \\
\text { Camera controller } \\
\text { (C2741-62) }\end{array}$ & Molecular Devices, Sunnyvale, USA \\
$\begin{array}{l}\text { CCD camera } \\
\text { (XC-ST70CE) }\end{array}$ & Hamamatsu, Herrsching am Ammersee, Germany \\
$\begin{array}{l}\text { Centrifuge (Rotilabo) } \\
\text { Computer Interface } \\
\text { (ITC-18) }\end{array}$ & Carl Roth, Karlsruhe, Germany \\
$\begin{array}{l}\text { Digital thermometer } \\
\text { (Voltcraft PL-120-T1) } \\
\text { electrophoresis power } \\
\text { supply (Consort EV231) }\end{array}$ & Conrad, Hirschau, Germany \\
Headstage (CV-7B) & Molecular Devices, Sunnyvale, USA
\end{tabular}


Table 2.2. List of devices

\begin{tabular}{|c|c|}
\hline Device & Supplier \\
\hline $\begin{array}{l}\text { Heated perfusion tube } \\
\text { (HPT-2) }\end{array}$ & alascience, Farmingdale, USA \\
\hline INTAS imaging system & $\begin{array}{l}\text { INTAS Science Imaging Instruments, Götingen, Ger- } \\
\text { many }\end{array}$ \\
\hline $\begin{array}{l}\text { Micropipette puller } \\
\text { (P-1000) }\end{array}$ & Sutter Instrument, Novato, USA \\
\hline Microscope (BX51WI) & Olympus, Hamburg, Germany \\
\hline $\begin{array}{l}\text { Mircromanipulator } \\
\text { (MP-225) }\end{array}$ & Sutter Instrument, Novato, USA \\
\hline Monitor (MX-15A) & ABUS Security-Center, Affing, Germany \\
\hline Osmometer (Vapro 5520) & Wescor, Logan, USA \\
\hline $\begin{array}{l}\text { PCR machine } \\
\text { (Mastercycler Pro S) }\end{array}$ & Eppendorf, Wesseling-Berzdorf, Germany \\
\hline $\begin{array}{l}\text { pH meter (Professional } \\
\text { Meter PP-15) }\end{array}$ & Sartorius, Götingen, Germany \\
\hline Polyimide heater & Minco, Aston, France \\
\hline $\begin{array}{l}\text { Pump (SR } 25 \text { 65rpm } \\
\text { Novoprene) }\end{array}$ & $\begin{array}{l}\text { Gardner Denver Thomas, Fürstenfeldbruck, Ger- } \\
\text { many }\end{array}$ \\
\hline Shaker (ThermoMixer & Eppendorf, Wesseling-Berzdorf, Germany \\
\hline $\begin{array}{l}\text { Comfort) } \\
\text { Silver wire (AG-10W) }\end{array}$ & Science Products, Hofheim, Germany \\
\hline $\begin{array}{l}\text { Stimulus isolator } \\
\text { (ISO-Flex) }\end{array}$ & A.M.P.I., Jerusalem , Israel \\
\hline $\begin{array}{l}\text { Temperature controller } \\
\text { (TC-20) }\end{array}$ & npi electronic, Tamm, Germany \\
\hline $\begin{array}{l}(\mathrm{TC}-20) \\
\text { valve controllers (VS-01H } \\
\text { and VS-01HL) }\end{array}$ & npi electronic, Tamm, Germany \\
\hline Vibratome (VT1200S) & Leica, Wetzlar, Germany \\
\hline $\begin{array}{l}\text { Video-to-USB Converter } \\
\text { (DFG/USB2-It) }\end{array}$ & The Imaging Source Europe, Bremen, Germany \\
\hline
\end{tabular}

Table 2.3. List of other materials

\begin{tabular}{ll}
\hline Item & Supplier \\
\hline $\begin{array}{l}\text { Borosilicate glass pipette } \\
\text { (KG-33) }\end{array}$ & King Precision Glass, Claremont, USA \\
$\begin{array}{l}\text { Cyanoacrylate glue } \\
\text { (Loctite 401) }\end{array}$ & Henkel Corp, Dublin, Ireland \\
$\begin{array}{l}\text { Intravenous flow } \\
\text { controller (Dial-a-flo) }\end{array}$ & Hospira, Sligo, Ireland \\
\end{tabular}


Table 2.3. List of other materials

\begin{tabular}{ll}
\hline Item & Supplier \\
\hline Plastic tubings & Tygon, Charny, France \\
Razor blade (Personna) & Edgewell Personal Care, St. Louis, Germany \\
$\begin{array}{l}\text { Septum Theta capillary }( \\
\text { TST150-6) }\end{array}$ & World Precision Instruments, Sarasota, USA \\
$\begin{array}{l}\text { Silver-silver chloride } \\
\text { pellet (E-201) }\end{array}$ & Science Products, Hofheim, Germany \\
$\begin{array}{l}\text { Single-use cannula, blunt } \\
(0.80 \times 22 \mathrm{~mm})\end{array}$ & B Braun Melsungen, Melsungen, Germany \\
\hline
\end{tabular}

Table 2.4. List of softwares

\begin{tabular}{ll}
\hline Software & Company \\
\hline Axograph & Axon Instrument, Foster City, USA \\
GraphPad Prism & GraphPad 6, La Jolla, USA \\
Igor Pro 6.1.2.0 & WaveMetrics, Lake Oswego, USA \\
INTAS imaging system & Göttingen, Germany \\
software & \\
Mini Analysis & Synaptosoft Inc., Fort Lee, USA \\
Multiclamp 700B & Axon Instrument, Foster City, USA \\
Commander & \\
\hline
\end{tabular}




\subsection{Animals}

\subsubsection{Genetic background}

PSD-95 KO mice were generated by the deletion of the guanylate kinase (GK) domain of PSD-95 as described in previous studies Abbas et al. 2009, Yao et al. 2004. The mutant line was backcrossed with mice of C57Bl6/J background (Charles River) for more than 10 generations. WT control and KO mutant mice were bred from heterozygous parents. Some KO mutant mice were bred from heterozygous females and KO mutant males. In mutant mice, PSD-95 mRNA is almost absence (5.7\% of WT levels) and PSD-95 protein was not detected with antibodies against $\mathrm{N}$ terminal (amino acid residues 77-299) and the PDZ3 domain (amino acid residues 353-504) of PSD-95 (Yao et al. 2004).

\subsubsection{Housing conditions and handling}

Mice were housed in the animal facility (European Neuroscience Institute, Göttingen) under standard conditions with 12-hour light/dark cycle. Maximum five mice were housed together per cage of 17 X $33 \mathrm{~cm}$. Access to food and water was provided ad libitum. Used cages were usually replaced once a week. Mice were weaned at around P21. Males and females were separated at weaning. For numbering, ear holes were punched at weaning, with some mice having paws tattooed before/around P5. The tip of the tail (1-2 mm) was cut for genotyping at either before/around P5 or at weaning. Mice before weaning were used shortly after they were transfered to the laboratory. Most mice after weaning were used shortly after the transfer, though a small number were used up to one day after the transfer while they were kept in the laboratory's scantainer under similar housing conditions as the animal facility. Opening of eyes was checked before the mouse was sacrificed to ensure that it matches the developmental stage of visual system. Male and female mice were selected randomly for all experiments. Animal caretakers have been changed during the study.

\subsubsection{Genotyping}

Genotyping was done before and after sacrificing the mice. 


\section{Tail lysis}

Mouse tails were lysed with lysis buffer consisting:

- PCR Buffer with Nonionic Detergents (PBND: 0.45\% Tween20, 0.45\% Nonident $\mathrm{P} 40,2.5 \mathrm{mM} \mathrm{MgCl} 2 \cdot 6 \mathrm{H}_{2} \mathrm{O}, 50 \mathrm{mM} \mathrm{KCl}, 10 \mathrm{mM}$ Tris and $0.1 \mathrm{mg} / \mathrm{mL}$ gelatine, $\mathrm{pH}$ 8.3)

- Proteinase K, 1:100 dilution

Tails were incubated in lysis buffer $\left(100 \mu \mathrm{L}\right.$ per $1-2 \mathrm{~mm}$ of tail) at $55^{\circ} \mathrm{C}$ for 3 hours to overnight with constant shaking. The samples were then heated to $99^{\circ} \mathrm{C}$ for 10 minutes to inactivate proteinase K. Supernatant was collected for storage after centrifugation at $14680 \mathrm{rpm}$ for 2 minutes. Tails were stored at $-20^{\circ} \mathrm{C}$ and lysed tail samples at $4^{\circ} \mathrm{C}$.

\section{polymerase Chain Reaction (PCR)}

Reaction mix for PCR was prepared as follow:

$\begin{array}{ll}\text { TNK buffer (10X) } & 2.2 \mu \mathrm{L} \\ \text { dNTP } & 2 \mu \mathrm{L} \\ \text { Forward primer, } 50 \mu \mathrm{m} & 0.2 \mu \mathrm{L} \\ \text { Reverse primer, } 50 \mu \mathrm{m} & 0.2 \mu \mathrm{L} \\ \text { dd }_{2} \mathrm{O} & 15.2 \mu \mathrm{L} \\ \text { Mango Taq Polymerase } & 0.2 \mu \mathrm{L}\end{array}$

The sequences of forward and reverse primers (5' to 3') for different genotypes (wild-type (WT) and mutant) are listed in table 2.5, $20 \mu \mathrm{L}$ of reaction mix was added to $2 \mu \mathrm{L}$ of lysed tail sample and PCR was run. The program for respective genes are shown in table 2.6 .

\section{Gel electrophoresis}

PCR product was analyzed with gel electrophoresis. 1\% agarose gel containing 5 mM sodium tetraborate and ethidium bromide (1\% stock, $15 \mu \mathrm{L}$ was added per litter 
Table 2.5. List of primers for genotyping

\begin{tabular}{ll}
\hline Gene & WT $^{*}$ Mutant* $^{*}$ \\
\hline PSD-95 & CAGGTGCTGCTGGAAGAAGG \\
& CTACCCTGTGATCCAGAGCTG \\
SAP97 & CCTCTACAGAATCTGAGTTGGCTC \\
& TAAGAAGGATCAACTGGCAAAGGTG \\
\hline
\end{tabular}

Notes: First sequence of each primer pair is the forward primer, second sequence is the reverse primer. Same pairs of primers were used for both WT and mutant knock-out (KO) genotypes of PSD-95 and SAP97.

Table 2.6. PCR programs

\begin{tabular}{ll}
\hline Gene & Program \\
\hline PSD-95 & $94^{\circ} \mathrm{C}(5 \mathrm{~min})$ \\
& 35 cycles for following steps: $94^{\circ} \mathrm{C}(45 \mathrm{~s}), 63^{\circ} \mathrm{C}(45 \mathrm{~s}), 72^{\circ} \mathrm{C}(1 \mathrm{~min})$ \\
& $72^{\circ} \mathrm{C}(10 \mathrm{~min})$ \\
SAP97 & $94^{\circ} \mathrm{C}(2 \mathrm{~min})$ \\
& 35 cycles for following steps: $94^{\circ} \mathrm{C}(30 \mathrm{~s}), 60^{\circ} \mathrm{C}(45 \mathrm{~s}), 72^{\circ} \mathrm{C}(1 \mathrm{~min})$ \\
& $72^{\circ} \mathrm{C}(4 \mathrm{~min})$ \\
\hline
\end{tabular}

Note: Reactions were cooled down to $4^{\circ} \mathrm{C}$ after PCR.

of agarose solution). $10 \mu \mathrm{L}$ of loading dye containing $5 \mathrm{mM}$ sodium tetraborate, $60 \%$ glycerol and bromophenol blue was added to $20 \mu \mathrm{L}$ of PCR product. The PCR product was loaded to the agarose gel and electrophoresis was run in $5 \mathrm{mM}$ sodium tetraborate buffer at $145 \mathrm{~V}$ for 30 to 45 minutes. Image of the gel was acquired with UV illumination by INTAS imaging system. The sizes of bands on the gel for different genotypes are listed in table 2.7 .

Table 2.7. Expected sizes of gel bands

\begin{tabular}{lcc}
\hline Gene & WT band (bp) & Mutant band (bp) \\
\hline PSD-95 & 255 & 355 \\
SAP97 & 600 & 900 \\
\hline
\end{tabular}




\subsection{Solutions for electrophysiology}

\section{Cutting solution}

Choline chloride $119 \mathrm{mM}$, kynurenic acid $1 \mathrm{mM}$, sodium ascorbate $1.3 \mathrm{mM}$, sodium pyruvate $3 \mathrm{mM}, \mathrm{NaHCO}_{3} 26 \mathrm{mM}$, glucose $30 \mathrm{mM}, \mathrm{KCl} 2.5 \mathrm{mM}, \mathrm{NaH}_{2} \mathrm{PO}_{4} 1 \mathrm{mM}$, $\mathrm{MgSO}_{4} \cdot \mathrm{H}_{2} \mathrm{O} 7 \mathrm{mM}, \mathrm{CaCl}_{2} \cdot 2 \mathrm{H}_{2} \mathrm{O} 1 \mathrm{mM}$.

artificial cerebrospinal fluid (ACSF)

$\mathrm{NaCl} 119 \mathrm{mM}, \mathrm{NaHCO}_{3} 26 \mathrm{mM}$, glucose $11 \mathrm{mM}, \mathrm{KCl} 2.5 \mathrm{mM}, \mathrm{NaH}_{2} \mathrm{PO}_{4} 1 \mathrm{mM}$, $\mathrm{MgSO}_{4} \cdot \mathrm{H}_{2} \mathrm{O} 1.3 \mathrm{mM}, \mathrm{CaCl}_{2} \cdot 2 \mathrm{H}_{2} \mathrm{O} 2.5 \mathrm{mM}$.For recording. $50 \mu \mathrm{M}$ of picrotoxin was added during recording to block GABA receptor-mediated current. See Section 2.5.4 to 2.5.7 for information about other drugs applied during recording.

\section{Recovery solution}

ACSF supplemented with $1 \mathrm{mM}$ of kynurenic acid was used.

\section{Intracellular solution}

Cesium gluconate-based: Cesium gluconate 120 mM, HEPES 20 mM, EGTA 0.4 $\mathrm{mM}, \mathrm{QX}_{314} \mathrm{Cl} 4 \mathrm{mM}$, TEA-Cl 5 mM, MgATP $4 \mathrm{mM}$, NaGTP $0.3 \mathrm{mM}$. Adjusted to $\mathrm{pH}$ 7.2-7.3 with $\mathrm{CsOH}(50 \% \mathrm{w} / \mathrm{v})$; adjusted to 289-295 Osm with $\mathrm{ddH}_{2} \mathrm{O}$.

Cesium methanesulfonate-based: $\mathrm{MeSO}_{3} \mathrm{H} 117.5 \mathrm{mM}, \mathrm{CsCl} 17.75 \mathrm{mM}$, HEPES $10 \mathrm{mM}$, EGTA $0.25 \mathrm{mM}$, glucose $10 \mathrm{mM}, \mathrm{MgCl}_{2} \cdot 6 \mathrm{H}_{2} \mathrm{O} 2 \mathrm{mM}, \mathrm{QX}_{314} \mathrm{Cl} 5 \mathrm{mM}$, TEA-Cl $10 \mathrm{mM}, \mathrm{Na}_{2} \mathrm{ATP} 4 \mathrm{mM}$, NaGTP 0.3 mM. Adjusted to pH 7.0-7.3 with $\mathrm{CsOH}$ (50\% w/v); adjusted to 290-293 Osm with $\mathrm{ddH}_{2} \mathrm{O}$.

Intracellular solution was prepared at $4^{\circ} \mathrm{C}$, filtered with $0.2 \mu \mathrm{M}$ filter and stored at $-80^{\circ} \mathrm{C}$. It was kept at $4^{\circ} \mathrm{C}$ during experiment. 


\section{Stock solutions}

Cutting solution and ACSF were prepared freshly with the following stock solutions: 10X cutting solution stock ( $\mathrm{KCl} 25 \mathrm{mM}, \mathrm{NaH}_{2} \mathrm{PO}_{4} 10 \mathrm{mM}, \mathrm{MgSO}_{4} \cdot \mathrm{H}_{2} \mathrm{O} 40 \mathrm{mM}$ ), 10X ACSF stock ( $\mathrm{NaCl} 1.19 \mathrm{M}, \mathrm{KCl} 25 \mathrm{mM}, \mathrm{NaH}_{2} \mathrm{PO}_{4} 10 \mathrm{mM}$ ), $\mathrm{MgSO}_{4} \cdot \mathrm{H}_{2} \mathrm{O} 1 \mathrm{M}$, $\mathrm{CaCl}_{2} \cdot 2 \mathrm{H}_{2} \mathrm{O} 1 \mathrm{M}$. All stock solutions were kept at room temperature.

\subsection{Acute slice preparation}

Mice were anesthetized with isoflurane and were killed by decapitation. The brain was isolated from the head and was cooled down in ice-cold cutting solution for approximately 1 minute. Anterior and posterior parts of the brain were removed and the remaining part containing the visual cortex was glued to the cutting stage with cyanoacrylate glue. The brain part was supported by an agarose $(2 \%)$ block. Left and right hemispheres were separated. 300 or $400 \mu \mathrm{m}$ thick coronal slices were cut from the dorsal end of the brain in ice-cold cutting solution with a vibratome. The slices containing visual cortex were transfered to the recovery solution at $35^{\circ} \mathrm{C}$ with continuous supply of carbogen ( $5 \%$ carbon dioxide and $95 \%$ oxygen). The slices were allowed to recover at $35^{\circ} \mathrm{C}$ for 45 minutes to 1 hour and then at room temperature in ACSF (without kynurenic acid supplement) for at least 45 minutes before recording.

\subsection{Electrophysiology}

\subsubsection{The setup}

\section{Recording chamber, ACSF perfusion and optics}

The recording chamber was attached to a movable stage which was installed under the objective of an upright microscope. ACSF perfusion inlet and outlet are attached to two sides of the chamber. The solution flowed into the chamber by gravity and was heated through a heated perfusion tube and by a polyimide heater located at the base of the recording chamber. The perfusion tube and the heater temperature 
was set by a temperature controller. A peristaltic pump removed the excess solution when the chamber was filled. The flow rate was regulated by an intravenous flow controller. $4 \mathrm{x}$ and $60 \mathrm{x}$ objectives were used. Live image was captured with a CCD camera and digitalized with a video-to-USB converter when required.

\section{Local perfusion system}

Homemade local perfusion system was used for the application of AMPA (Section 2.5.4 and 2.5.7). The drug outlet is made from a blunt cannula (inner diameter: $0.80 \mathrm{~mm}$ ), which has the narrowest diameter in the system. The drug outlet was placed horizontally at ca. $1.16 \mathrm{~mm}$ to the left of the recording pipette, close to slice surface as much as possible without contacting, and ca. $+45^{\circ}$ to the flow of ACSF perfusion. The centre of the drug outlet was aligned with the position of the recording pipette. The flow of the local perfusion was ca. $1.7 \mathrm{ml} / \mathrm{min}$.

\section{Electrodes, headstage and amplifier}

The recording electrode is made of silver wire and is connected to a headstage.The wire was chlorinated with $2 \mathrm{M}$ of potassium chloride by electrolysis. A recording pipette filled with intracellular solution was secured to the pipette holder of the headstage. The pipette is made of borosilicate glass (Outer/internal diameter: $1.50 / 1.00 \mathrm{~mm}$ ) with tip resistance of ca. 2.5 to $5 \mathrm{M} \Omega$. The pipette holder is connected to a $10 \mathrm{ml}$-syringe and a mouth piece for the application of pressure. The stimulation electrodes (bipolar: cathode and anode; made of silver wires) connected to a stimulus isolator and were inserted into a Septum Theta capillary pipette (Outer/internal diameter: 1.50/1.02 mm) filled with ACSF. The pipettes for recording and stimulation were made with a micropipette puller. The position of the recording pipette was controlled through a micromanipulator and the stimulation pipette was controlled manually. The headstage was grounded to the ASCF bath by placing a silver-silver chloride pellet (diameter/length: $2.0 / 4.0 \mathrm{~mm}$ ) pellet inside the bath. A computer-controlled amplifier with feedback resistor set to $500 \mathrm{M} \Omega$ was used. 


\section{Computer interface and softwares}

A computer interface between the amplifier and the computer converts analogue signals to/from digital signals. The amplifier is controlled through the software

MultiClamp 700B Commander. Signals were recorded with the software Igor Pro 6.1.2.0 (user-defined procedure files for electrophysiology were programmed by Dr. Juliane Marie Krüger).

\section{Assembly of the devices}

The microscope, recording chamber and the headstage are fixed on a table mounted on air legs which provide passive vibration isolation. The table is enclosed by a Faraday cage to shield the recording devices from the electromagnetic fields in the environment.

\subsubsection{General recording configuration}

Electrophysiological recording was conducted with continuous perfusion of ACSF with a flow rate of $2.8 \pm 0.3 \mathrm{~mL} /$ minute. ACSF was supplied with carbogen (5\% carbon dioxide and $95 \%$ oxygen) continuously and maintained at 29.5 to $31^{\circ} \mathrm{C}$. The volume of ASCF in the recording chamber was maintained at ca. $2.5 \mathrm{~mL}$, with excess ACSF recycled if no additional drugs were applied. An acute slice was secured in the chamber by an U-shaped platinum harp with synthetic threads $(88 \%$ polyamide, $12 \%$ elasthane) pressing down the slice. For whole-cell voltage-clamp recording, a positive pressure (ca. 20\% higher than atmospheric pressure) was applied to the recording pipette and the pipette was placed near the target neuron. The pipette current was zeroed with the automated pipette offset function of the amplifier. The positive pressure was released when the pipette was pressing against the neuron. The holding voltage was switched to $-60 \mathrm{mV}$ when a gigaseal was formed. Whole-cell configuration was achieved by breaking the cell membrane with pulses of strong mouth suction. A $20 \mathrm{~ms}$ test pulse of $-5 \mathrm{mV}$ was applied to each sweep (a unit of recording). Signals from the amplifier were digitalized at $10 \mathrm{kHz}$ and filtered at $3 \mathrm{KHz}$. The recording was monitored online and analyzed offline. Each slice was used for more than one recording unless otherwise specified. For specific protocols of individual experiments, please see the following sections. 


\section{Target neurons}

Layer 2/3 of primary visual cortex (V1) was studied. Pyramidal neurons were recorded randomly.

\section{Stimulus intensity and electrode position}

The stimulation electrode was placed at layer 4 of V1, near the longitudinal axis of the patched cell. The electrode position might be adjusted to nearby positions after patching to increase the evoked response or to avoid the induction of polysynaptic response. Stimulus intensity was adjusted to a magnitude (usually in $\mu \mathrm{A}$ range) which could evoke responses with high signal-to-noise ratio (AMPAR-EPSCs and the NMDAR component of EPSCs at $+40 \mathrm{mV}$ were both greater than $10 \mathrm{pA}$ ), while did not induce epileptic responses. Stimulus intensity was tested at $-60 \mathrm{mV}$ for AMPAR-EPSCs and at positive holding voltage when NBQX was present. Stimulus intensity was tested with at least $5 \mathrm{~s}$ interval at $-60 \mathrm{mV}$ or $20 \mathrm{~s}$ interval at $+40 \mathrm{mV}$ to avoid the induction of plasticity. Same stimulus intensity and electrode position were maintained during each recording.

\section{Quality control for reliable recording}

Before recording, at least 5 min was allowed for the slice to accommodate to the recording condition after being transfered to the recording chamber. Recording was started at least 5 min after breaking the cell to allow the diffusion of intracellular solution and the formation of a better seal. This also allowed time for the electrical properties of the patched cell to stabilize. To achieve a better seal, pulses of suction or positive pressure might be applied to the cell after breaking the cell. Series resistance was monitored online. Recordings with series resistance greater than $30 \mathrm{M} \Omega$ or change greater than $20 \%$ were paused and were resumed when the resistance was improved. Noise level was also monitored online. If the noise level was visibly higher than usual, more sweeps would be recorded if possible (allowing noise reduction by averaging); for mEPSC recording, recording would be terminated. 


\subsubsection{Evoked excitatory postsynaptic current (EPSC)}

Cells were held at $-60 \mathrm{mV}$ for the recording of AMPAR-mediated EPSC (AMPAREPSC) and at $+40 \mathrm{mV}$ for NMDAR-mediated EPSC (NMDAR-EPSC). EPSC was evoked with $200 \mu$ s-long electric stimulus applied at $25 \mathrm{~ms}$ after the test pulse. Sweeps of $250 \mathrm{~ms}$ long each were recorded, with one stimulus per sweep. Intertrial interval (time interval between two sweeps) was $5 \mathrm{~s}$. For the recording of AMPAR/NMDAR ratio (ratio of AMPAR-EPSC to NMDAR-EPSC, AMPAR-EPSCs were recorded before NMDAR-EPSCs. Other experiments involving recording of evoked EPSCs follow the same protocol unless otherwise specified.

\subsubsection{Miniature EPSC (mEPSC)}

Cells were held at $-60 \mathrm{mV}$ to record AMPAR-mediated mEPSC in the absence of electric stimulation. $500 \mathrm{nM}$ of tetrodotoxin (TTX) was added to ACSF to eliminate spontaneous action potential firing via blocking voltage-gated sodium channels. Sweeps of $10 \mathrm{~s}$ long each were recorded. Each slice was used for multiple recording. For experiments involving AMPA treatment, $2.5 \mu \mathrm{M}$ of AMPA was applied locally (see Section 2.5.1) to the vicinity of the patched cell for $20 \mathrm{~s}$, after the recording of basal mEPSCs. mEPSCs 3 min after the end of AMPA perfusion was used for the assessment of the drug effect. Each slice was used for only one recording with AMPA treatment. For experiments involving trichlormethiazide (TCM) treatment, $500 \mu \mathrm{M}$ of TCM was washed into the recording chamber by perfusion after the recording of basal mEPSCs. mEPSCs recorded 2 min after washing in TCM were used for the assessment of the drug effect. TCM was washed out for 3 min and the same slice was used for another recording with TCM treatment. Each slice was used for at most two recordings with TCM treatment.

\subsubsection{Paired-pulse ratio recording}

For each sweep, two pulses of EPSCs were evoked with paired stimuli of 50 or 100 $\mathrm{ms}$ interval. Cells were held at $-60 \mathrm{mV}$ for the recording of paired pulses of AMPAREPSCs. Paired pulses of NMDAR-EPSCs were recorded at $+40 \mathrm{mV}$ in the presence of $5 \mu \mathrm{M}$ of 2,3-dihydroxy-6-nitro-7-sulfamoyl-benzo[f] quinoxaline-2,3-dione (NBQX) (an AMPAR antagonist). Each sweep was $250 \mathrm{~ms}$ long and the intertrial interval was $5 \mathrm{~s}$ or $20 \mathrm{~s}$. Different slices were used for recordings with $5 \mathrm{~s}$ and $20 \mathrm{~s}$ intertrial interval. 


\subsubsection{NMDAR blocking by MK-801}

Cells were held at $+40 \mathrm{mV}$. Evoked NMDAR-EPSCs were recorded in the presence of $5 \mu \mathrm{M}$ of NBQX (AMPAR antagonist) and $5 \mu \mathrm{M}$ of MK-801 use-dependent NMDAR blocker: blocks only the activated NMDARs). 50 sweeps were recorded and the intertrial interval was $10 \mathrm{~s}$. Each slice was used for only one recording. $500 \mu \mathrm{M}$ of trichlormethiazide (TCM) was added additionally to test the potential presynaptic effect of the drug.

\subsubsection{AMPA response}

Cells were held at $-60 \mathrm{mV}$. 2.5 $\mu \mathrm{M}$ of AMPA was applied locally (see Section 2.5.1) to the vicinity of the patched cell for $20 \mathrm{~s}$. The membrane current induced by AMPA was recorded in the presence of $500 \mathrm{nM}$ of TTX. ACSF was not recycled during the application of AMPA. Each slice was used for only one recording.

\subsection{Data analysis}

\subsubsection{Evoked EPSC and AMPAR/NMDAR ratio}

EPSC amplitude was measured by the software Igor Pro. The amplitude of an evoked AMPAR-EPSC response at $-60 \mathrm{mV}$ is defined as the difference between the holding current before the stimulation (baseline) and the inward current peak evoked by the stimulation. NMDAR component of an evoked EPSC response at $+40 \mathrm{mV}$ is defined as the difference between the baseline and the outward current at $60 \mathrm{~ms}$ after the peak. For recordings in the presence of NBQX, the amplitude of an evoked NMDAR-EPSC response at $+40 \mathrm{mV}$ is defined as the different between the baseline and the outward current peak. The baseline and the peak positions of the averaged response of each recording were used for the calculation of the amplitudes of all the responses in the recording. AMPAR/NMDAR ratio of a recording is the ratio of the absolute average amplitude of the AMPAR-EPSCs at $-60 \mathrm{mV}$ to the average amplitude of the NMDAR component of the EPSCs at +40 $\mathrm{mV}$. The distribution of such ratio is skewed. To avoid errors in statistical analysis, logarithm transformation of the data was performed before statistical analysis. Geometric mean (natural exponential value of the mean natural logarithm of each data point) was presented in the results of AMPAR/NMDAR ratio. 


\subsection{2 mEPSC}

Sweeps of each recording were concatenated with the software Igor Pro, excluding the test pulse in each sweep. 150 to 200 mEPSC events per recording were detected semi-manually with the software Mini Analysis: a possible event was identified manually if the rise time was visibly shorter than the decay time and the event decayed non-linearly. The event was then selected by the cursor in the software. The software determined the baseline and the peak position of the event according the following criteria (relative to the cursor):

Time period to find a local event peak $(\mu \mathrm{s}): 5000$

number of data point to average peak: 3

Peak direction: negative (for inward current)

Time point before the peak where calculation of average baseline starts $(\mu \mathrm{s}): 20000$ Time period used for calculation of average baseline: 10000

Maximum time period after the peak to calculate decay time: 10000

Fraction of peak amplitude at where decay ends: 0.3

The event amplitude was calculated by subtracting the average baseline from the local event peak. The $10-90 \%$ rise time is the difference between the time point at $10 \%$ and the time point at $90 \%$ of the peak amplitude. The decay time is the difference between the time point at the peak and the time point where the decay ends (as defined by the fraction of the peak amplitude). The area under an event was calculated by taking the integral of amplitude from the peak to the point where the decay ends. Real event peaks and noise peaks were distinguished by the amplitude and the area under the events. Events with amplitude of $7 \mathrm{pA}$ or above, and area of 11 (arbitrary unit) were accepted as real event peaks by the software.

\subsubsection{PPR}

The PPR is defined as the ratio of the amplitude of the second evoked EPSC response to that of the first response (calculation of amplitude: section 2.6.1). Responses at $-60 \mathrm{mV}$ were used for the calculation of the paired-pulse ratio of AMPAR-EPSCs (AMPAR-PPR) and responses at $+40 \mathrm{mV}$ for the paired-pulse ratio of NMDAR-EPSCs (NMDAR-PPR), The PPR for each recording is the average of the PPR for each sweep in the recording. 


\subsubsection{NMDAR blocking by MK-801}

Amplitudes of all evoked NMDAR-EPSC responses were normalized to the first response in the same recording. The decay in NMDAR-EPSC amplitude were fitted to the two-phase exponential decay (double exponential decay) model by least squares fitting with the software Prism:

$$
A=A_{\text {final }}+A_{\text {fast }} \cdot \exp \left(-K_{\text {fast }} \cdot t\right)+A_{\text {slow }} \cdot \exp \left(-K_{\text {slow }} \cdot t\right)
$$

where

$$
\begin{aligned}
& A_{\text {fast }}=\left(A_{\mathrm{o}}-A_{\text {final }}\right) \cdot \text { Percent }_{\text {fast }} \\
& A_{\text {slow }}=\left(A_{\mathrm{o}}-A_{\text {final }}\right) \cdot\left(100 \%-\text { Percent }_{\text {fast }}\right)
\end{aligned}
$$

$A$ is the amplitude; $A_{\text {final }}, A_{\text {fast }}, A_{\text {slow }}$ and $A_{o}$ are the amplitude at infinite time, of fast component, of slow component and the initial amplitude (i.e. at time $=0$ ) respectively; $\mathrm{K}_{\text {fast }}$ and $\mathrm{K}_{\text {slow }}$ are the rate constants of the fast and slow component respectively; $t$ is the time; Percent $_{\text {fast }}$ is the percentage of fast component. $A_{\text {final }}$ is assumed to be zero.

Two components (fast and slow) of the decay were resolved by this model. To obtain $\mathrm{K}_{\text {fast }}$ and $\mathrm{K}_{\text {slow }}$, exponential fitting was performed with the value of Percent $\mathrm{f}_{\text {fast }}$ constrained to the percentage of silent synapses (57.1\% for P13 wild-type mice, value from Dr. Yuzhang Liu, Pittsburgh (unpublished); 26.91\% for P30 wild-type mice, value from Huang et al. (2015). Time constants $\tau_{\text {fast }}$ and $\tau_{\text {slow }}$ are the reciprocals of $\mathrm{K}_{\text {fast }}$ and $\mathrm{K}_{\text {slow }}$ respectively. To estimate Percent fast $_{\text {for each recording, fitting }}$ was repeated with $\mathrm{K}_{\text {fast }}$ and $\mathrm{K}_{\text {slow }}$ constrained to the values obtained previously for each recording.

\subsubsection{AMPA-induced current}

The amplitude of an AMPA-induced inward current is defined as the difference between the holding current before the application of AMPA and the largest inward current during the drug application. The amplitude was measured with the software AxoGraph. 


\section{Results}

\subsection{Developmental changes in synaptic transmission and homeostasis of AMPAR-mEPSC frequency}

Huang et al. 2015) showed by minimal stimulation assay that silent synapse fraction decreases during development at selected time points: P10-12 (before eye opening), P25-30 and P60-70 (during and after the critical period for ocular dominance plasticity). Several possibilities may account for the decreased silent synapse fraction, e.g. the conversion of silent synapses to AMPAR+ synapses or by the pruning of silent synapses. In order to understand the role of silent synapse maturation in circuit refinement, it is essential to know the exact changes in silent synapse or AMPAR+ synapse number during development. However, the challenges here are that, silent synapses cannot be directly observed by electrophysiology, and minimal stimulation assay can only detect relative changes of the silent synapse fraction. Therefore, to evaluate changes in synapse number, I measured the change in AMPAR+ synapse number with AMPAR-mediated mEPSC (AMPAR-mEPSC) recording which detects spontaneous transmission at AMPAR+ synapses.

An mEPSC is a postsynaptic quantal response to an event of spontaneous vesicle release. The incidence of spontaneous release is so rare that, at most of the time, only one vesicle is released at one synapse at a time. Therefore, the mEPSC frequency is directly proportional to AMPAR+ synapse number. AMPAR-mEPSCs recorded here were mediated by AMPAR + synapses but not silent synapses. Whole-cell voltage clamp recording (figure 3.1 $\mathrm{A}$ ) was performed at $-60 \mathrm{mV}$ when NMDARs, which are found in both silent and AMPAR + synapses, remained closed and no currents passed through them. $500 \mathrm{nM}$ Tetrodotoxin (TTX) was added to block spontaneous action potential-triggered synaptic transmission to ensure that the responses recorded were purely driven by spontaneous vesicle release. The experiment was performed at P10 to P31 (before eye opening to near the end of the critical period), where most developmental changes occur. Considering developmental changes could happen rapidly, I performed the experiment with two additional time points: upon eye opening (p13-14) and at the start of critical period (P19-21). Besides, 
I used PSD-95 KO mice as a control which is known to have the silent synapse fraction preserved during development (Huang et al. 2015).

\subsubsection{AMPAR-mEPSC frequency increased upon eye opening but remained unchanged afterwards}

Decrease in silent synapse fraction might reflect an increase in AMPAR + synapse number. To check whether there were any changes in AMPAR + synapse number, I recorded AMPAR-mEPSCs and analyzed the frequency. I found the frequency increased upon eye opening (P13) but remained unchanged afterwards during critical period (P20 and P30) (figure B.1). Unexpectedly, the change in the frequency did not follow the trend of silent synapse fraction during the same development stages where the fraction was unchanged upon eye opening but decreased afterwards. This suggests that there was an increase in AMPAR + synapse number upon eye opening when the number reached at a certain pre-set value, and the number was kept stable dynamically afterwards, i.e. a homeostasis of AMPAR + synapse number and therefore a homeostasis of mEPSC frequency after eye opening.

In PSD-95 KO mice, the trend of change in AMPAR-mEPSC frequency was similar to that in WT mice although the silent synapse fraction remains at high level after eye opening (P13) (figure 3.1F). Interestingly, PSD-95 KO mice had lower frequency than WT mice not only at later developmental stages, but also at an age as early as P10 when PSD-95 expression in WT mice is very low. In addition, despite PSD-95 KO mice and WT mice have similar silent synapse fraction before eye opening (P10), KO mice had much lower AMPAR-mEPSC frequency (ca. $37 \%$ of that of WT mice). These results suggest that the developmental homeostasis of AMPAR number can be achieved in the absence of PSD-95, and that the homeostasis and the PSD-95-dependent silent synapse maturation operate independently.

Another important quantal property of synaptic transmission is the AMPAR-mEPSC amplitude, which is directly proportional to the quantal size. As mentioned in Introduction, changes in quantal size most likely reflects the change in postsynaptic AMPAR number in a single synapse. AMPAR-mEPSC amplitude was only slightly affected by the deletion of PSD-95 compared to the $\sim 37-47 \%$ decrease in the frequency (figure 3.1; statistic test: table A.2) despite the important role of PSD95 in silent synapse maturation. Besides, WT and PSD-95 mice had the same trend of changes during development: the amplitude decreased upon eye opening (P13) and remained unchanged afterwards during the critical period (P20 and P30). 

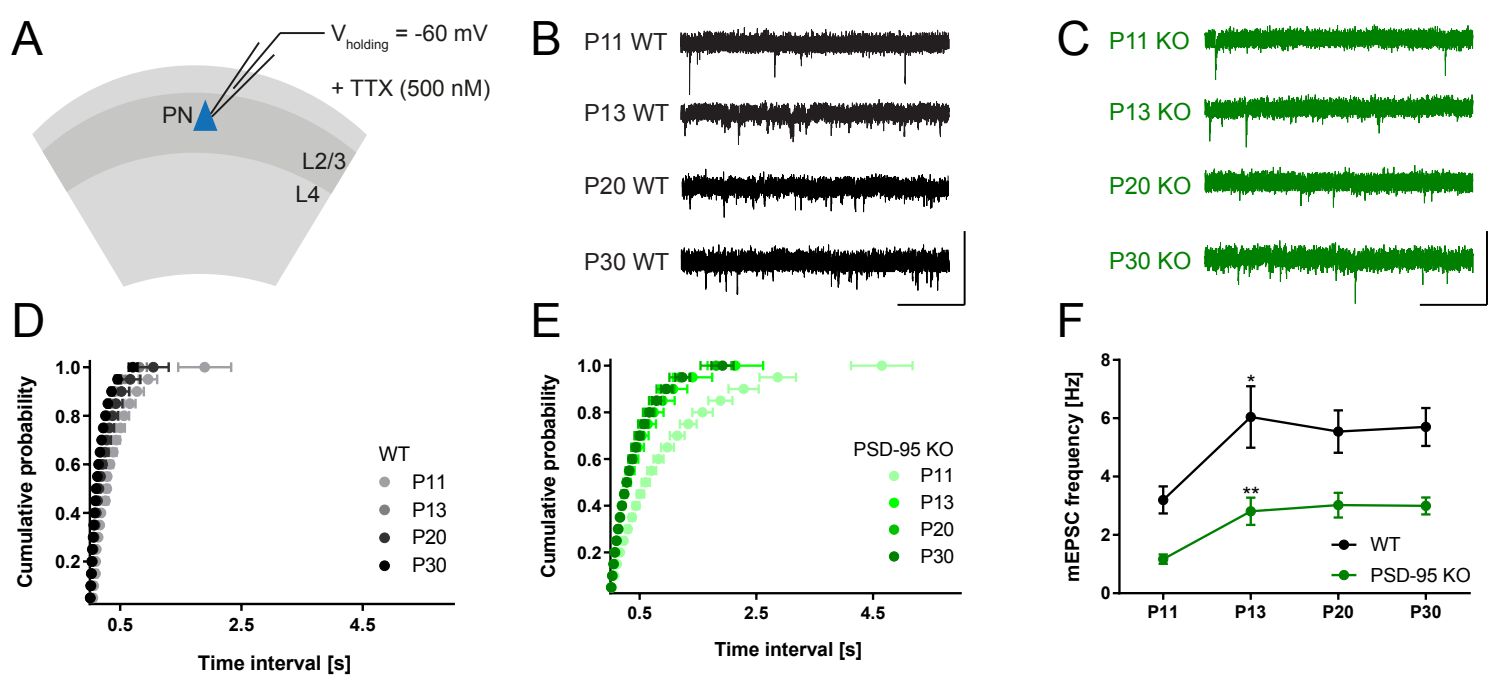

\section{$\mathrm{E}$}

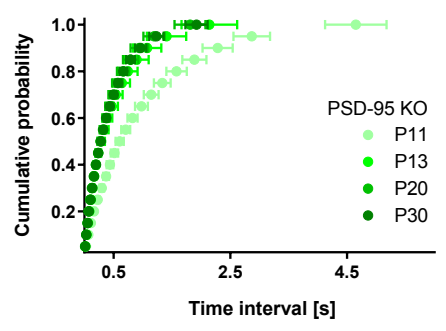

$\mathrm{F}$
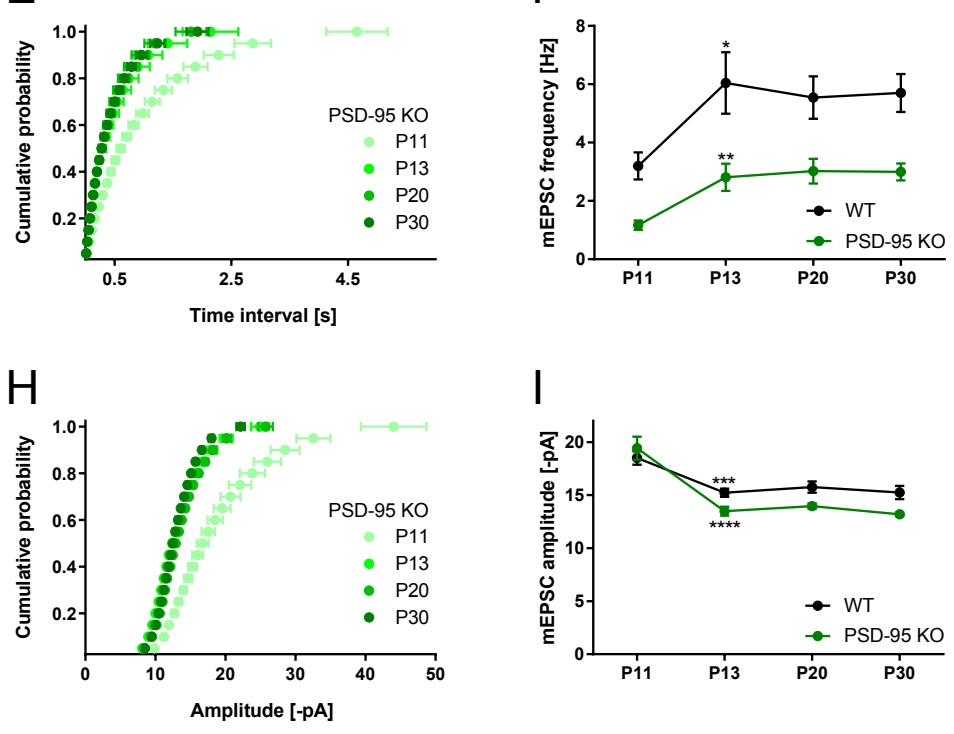

Fig. 3.1. Developmental changes in AMPAR-mEPSC frequency and amplitude of L2/3 pyramidal neurons of $\mathrm{V} 1$.

A: Schematic diagram of the recording configuration. PN: pyramidal neuron. Cells were voltage-clamped at $-60 \mathrm{mV}$ and mEPSCs of AMPAR + synapses (AMPARmEPSCs) were recorded in the presence of tetrodotoxin (TTX, $500 \mathrm{nM}$ ).

$\mathrm{B}$ and C: Example traces of mEPSC recording from wild-type (WT) and PSD-95 knockout (KO) mice at different ages. Scale bars: 50 pA, $500 \mathrm{~ms}$.

D and G: Cumulative probability of mEPSC time interval (D) and amplitude (G) for WT mice. Bin size: 20 events.

$\mathrm{E}$ and $\mathrm{H}$ : Cumulative probability of mEPSC time interval (E) and amplitude $(\mathrm{H})$ for PSD-95 KO mice. Bin size: 20 events.

F and I: Summary graphs of mEPSC frequency (F) and amplitude (I) during development in WT and PSD-95 KO mice. Frequency at P11 vs. P13 in WT: $\mathrm{P}=0.0284$; $\mathrm{P} 11$ vs. $\mathrm{P} 13$ in $\mathrm{PSD}-95 \mathrm{KO}: \mathrm{P}=0.0065$. Amplitude at $\mathrm{P} 11$ vs. $\mathrm{P} 13$ in WT: $\mathrm{P}=0.0002$; $\mathrm{P} 11$ vs. $\mathrm{P} 13$ in PSD-95 KO: $\mathrm{P}<0.0001$ (Welch's t-test).

Age: postnatal day ( \pm 1 day). Error bars: S.E.M. *p $<0.05 * * \mathrm{p}<0.01 * * * \mathrm{p}<$ 0.001 . 
This indicates that PSD-95 deletion did not affect the developmental change in quantal size. Taken together, these results suggest that PSD-95's role in synaptic transmission mainly lies in synapse maturation but not in the regulation of quantal size (i.e. postsynaptic AMPAR number at single synapses).

\subsubsection{AMPAR/NMDAR ratio increased during development}

The homeostasis of AMPAR-mEPSC frequency during the critical period described above predicts no change in AMPAR + synapse number. If there was no change in AMPAR + synapse number, the decrease in the silent synapse fraction should be caused by a decrease in silent synapse number. Since the change in AMPAR+ synapse number but not silent synapse number affects a neuron's excitatory input, I studied the developmental changes in excitatory input by measuring the evoked excitatory transmission. I recorded AMPAR-EPSCs of pyramidal neurons at layer 2/3 of V1 with electrical stimulation at layer 4 (figure 3.2A). Cell-to-cell variability and variation in experimental conditions (such as stimulus intensity and location) could affect the amplitude of AMPAR-EPSCs. In order to compare the results of different recordings, the amplitude of NMDAR-EPSCs was used as a reference and the results are presented as the AMPAR/NMDAR ratio (ratio of AMPAR-EPSC amplitude to NMDAR-EPSC amplitude; definition see section 2.6.1).

In WT mice, the AMPAR/NMDAR ratio increased during development from P10 (before eye opening) to P30 (near the end of critical period) (figure 3.2p). Given that the mEPSC amplitude was unchanged, the postsynaptic AMPAR number was likely unchanged and therefore the increase in AMPAR/NMDAR ratio should mainly be due to an increase in AMPAR + synapse number. This result is consistent with silent synapse maturation and indicates that evoked and spontaneous transmission are differently affected by the developmental processes. In the following sections, I will explore some of the potential mechanisms for the regulation of spontaneous transmission during development. 
A

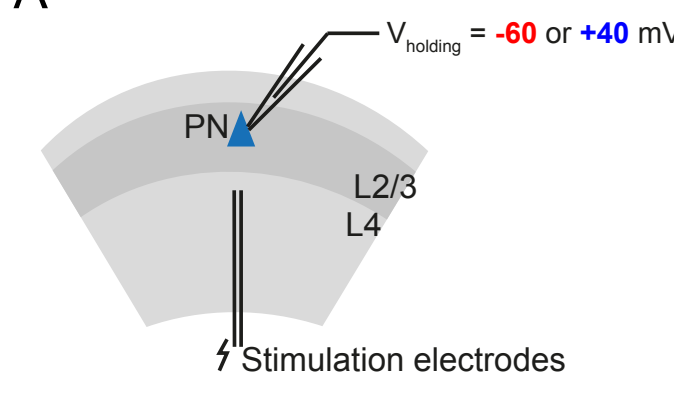

C

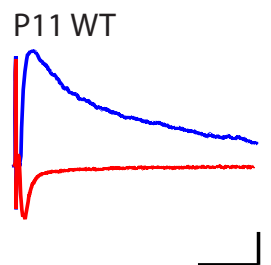

B

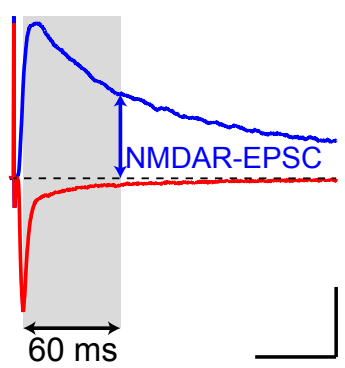

P13 WT

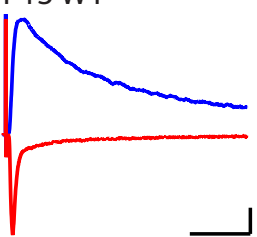

D

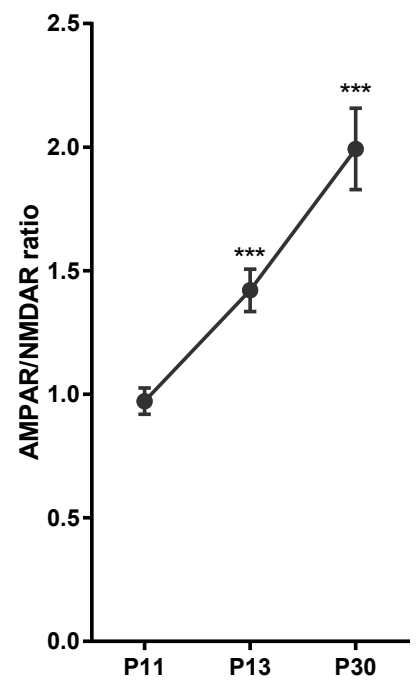

Fig. 3.2. AMPAR/NMDAR ratio in layer $2 / 3$ pyramidal neurons increased from $P 11$ to P30.

A: Schematic diagram of the recording configuration. PN: pyramidal neuron. Cells were voltage-clamped at -60 or $+40 \mathrm{mV}$ for the recording of AMPAR-EPSCs or NMDAR-EPSCs respectively.

$\mathrm{B}$ : AMPAR-EPSC decays to negligible level at $60 \mathrm{~ms}$ after the peak response (as shown in recording at $-60 \mathrm{mV}$, red). The EPSC at this time point was considered as solely mediated by NMDARs. NMDAR-EPSC was recorded at $+40 \mathrm{mV}$ (blue). Scale bars: $100 \mathrm{pA}, 50 \mathrm{~ms}$.

C: Example traces for wild-type (WT) mice at P11, P13 and P30. Red and blue: recording at $-60 \mathrm{mV}$ and $+40 \mathrm{mV}$ respectively. Scale bars: $100 \mathrm{pA}, 50 \mathrm{~ms}$.

D: Geometric means of AMPAR/NMDAR ratio at different age groups. P11 vs. $\mathrm{P} 13$ : $\mathrm{P}=0.0001$; $\mathrm{P} 13$ vs. $\mathrm{P} 30: \mathrm{P}=0.0020$ (Welch's t-test).

Age: postnatal day ( \pm 1 day). Error bars: S.E.M. $* *$ p $<0.001$. 


\subsection{Developmental change in presynaptic neurotransmitter release}

As shown in Section 3.1.1, the developmental change in AMPAR-mEPSC frequency (which is directly proportional to AMPAR + synapse number) is asymmetrical to the change in silent synapse fraction. However, besides synapse number, mEPSC frequency is also influenced by the vesicle release propensity, which is closely related to the release probability of neurotransmitter vesicles. Simultaneous decrease in release probability during development could happen to compensate for the increase in synapse number, accounting for the homeostasis of mEPSC frequency after eye opening (P13). To test if there are any developmental changes in release probability, I tested the change in release probability via two different electrophysiological experiments: paired-pulse ratio measurement and MK-801 induced NMDAR-EPSC decay.

\subsubsection{Paired-pulse ratio (PPR) of AMPAR-EPSC was unchanged}

Two identical stimuli in short time interval can lead to short-term depression or facilitation in EPSC amplitude, depending on the release probability of the synapse. If the release probability is high, the first stimulus can trigger the fusion of large amount of neurotransmitter vesicles, causing a depletion of the vesicles which leads to a smaller EPSC response to the second stimulus (i.e. paired-pulse depression). If the release probability is low, the accumulation of residual presynaptic calcium ions triggered by the first stimulus could cause a bigger EPSC response to the second stimulus (i.e. paired-pulse facilitation). Therefore, release probability is inversely proportional to the ratio of the second EPSC amplitude to the first EPSC amplitude (i.e. paired-pulse ratio (PPR) . PPR measurement allows one to detect the change in release probability. In theory, PPR is independent of postsynaptic receptors. Therefore, it can be measured by recording either AMPAR-EPSCs or NMDAR-EPSCs.

I first measured the paired-pulse ratio of AMPAR-EPSCs (AMPAR-PPR) at P11, P13 and P30 to check the possibility of a compensatory decrease in release probability in parallel to an increase in AMPAR+ synapse number (figure 3.3A). I found no developmental change in the AMPAR-PPR with both 50 and 100 ms stimulus 
interval (figure 3.3 C), suggesting that the release probability of AMPAR + synapses did not change during development. Note that PPR was the same with 50 and 100 ms stimulus interval even the time allowed for residual calcium clearance was doubled, suggesting that the influence of residual calcium ions on PPR might not be significant. Nevertheless, release probability could still influence PPR through other mechanisms of short term plasticity, such as the aforementioned vesicle depletion. To confirm whether there was a developmental change in release probability, I tested the change in release probability with a different approach, which will be discussed in in Section 3.2.3.

\subsubsection{Paired-pulse ratio (PPR) of NMDAR-EPSC increased}

Desensitization of AMPARs can cause a reduction in AMPAR-EPSC size. Therefore, the exchange rate of desensitized AMPARs can affect the AMPAR-PPR (Heine et al. 2008). Change in release probability during development might not be detected with AMPAR-PPR if there is a developmental change in the exchange rate of desensitized AMPARs. To validate the results of AMPAR-PPR, I additionally tested the paired-pulse ratio of NMDAR-EPSCs (NMDAR-PPR) which measures the PPR of NMDAR + synapses (i.e. essentially all glutamatergic synapses; figure 3.4A). Interestingly, NMDAR-PPR was lower than AMPAR-PPR at both age groups (figure 3.4D). One may expect that NMDAR-EPSCs have longer decay time than AMPAR-EPSCs, therefore while the first AMPAR response had already decayed at $100 \mathrm{~ms}$ when the second stimulus was triggered, the first NMDAR response had not completely decayed and would contribute to the amplitude of the second response. Consequently, a higher NMDAR-PPR would be expected. However, since the composite response of the first and the second stimulus was not bigger, this hightlights that the desensitized state of NMDARs lasts longer and/or the exchange rate for NMDARs is slower, leading to a lower NMDAR-PPR compared to AMPAR-PPR.

During development, NMDAR-PPR increased from P13 to P30, indicating that there was an increase in the average release probability at NMDAR+ synapses. NMDAR+ synapses include both silent synapses and AMPAR+ synapses, as both of these synapses mediate NMDAR-mediated transmission. Given that the PPR for AMPAR+ synapses was unchanged, the increase in NMDAR-PPR should be mainly mediated by some changes at silent synapses. For example, there might be a decrease in silent synapse release probability, or a decrease in silent synapse fraction if silent 


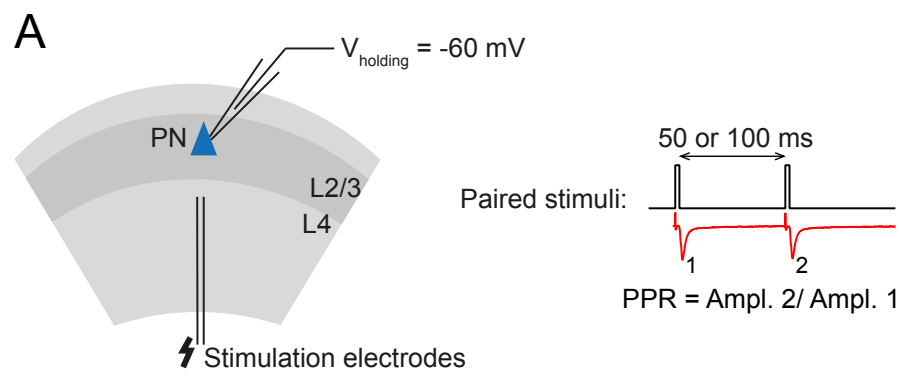

B $50 \mathrm{~ms}$ $100 \mathrm{~ms}$
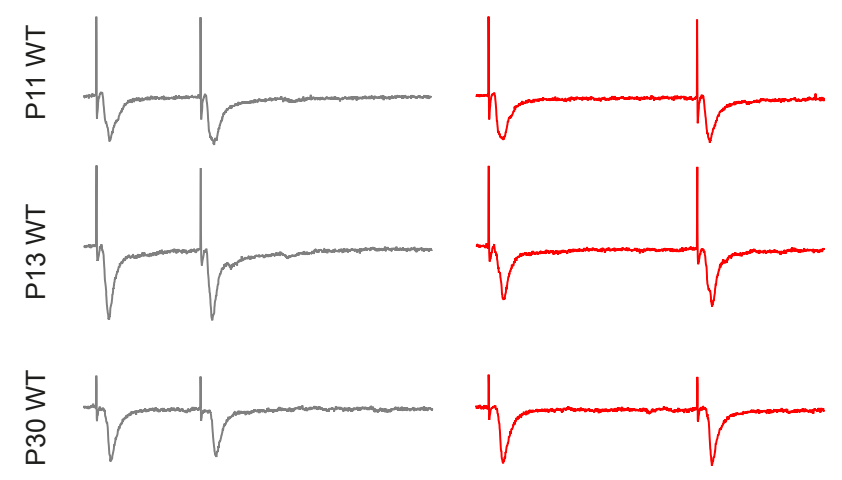

C

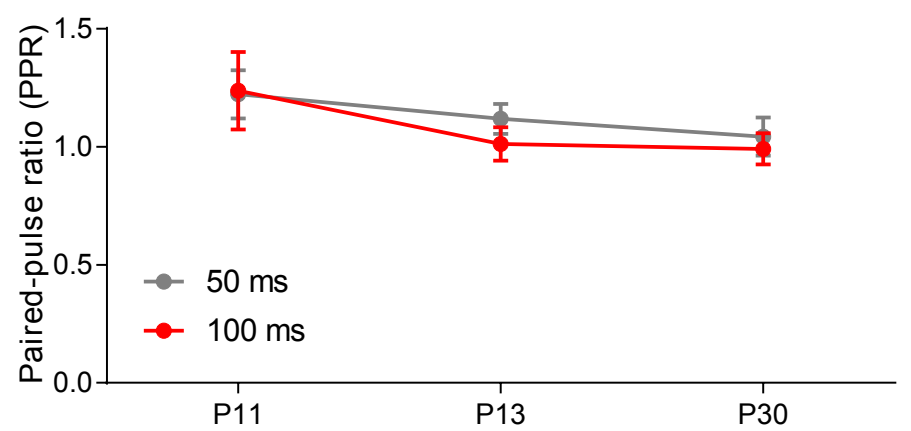

Fig. 3.3. Paired-pulse ratio (PPR) of AMPAR+ synapses with 50 or 100 ms stimulus interval was unchanged during development.

A: Schematic diagram of the recording configuration. PN: pyramidal neuron. Cells were voltage-clamped at $-60 \mathrm{mV}$ for the recording of AMPAR-EPSCs. Paired stimuli of 50 or $100 \mathrm{~ms}$ interval were applied.

B: Example traces of PPR recording with paired stimuli of $50 \mathrm{~ms}$ (grey) or 100 ms (red) from wild-type (WT) mice at P11, P13 and P30.

C: Means of PPR with different stimulus interval and at different age groups. Age: postnatal day ( \pm 1 day). Error bars: S.E.M. . Scale bar: 200 pA, 50 ms. 
A
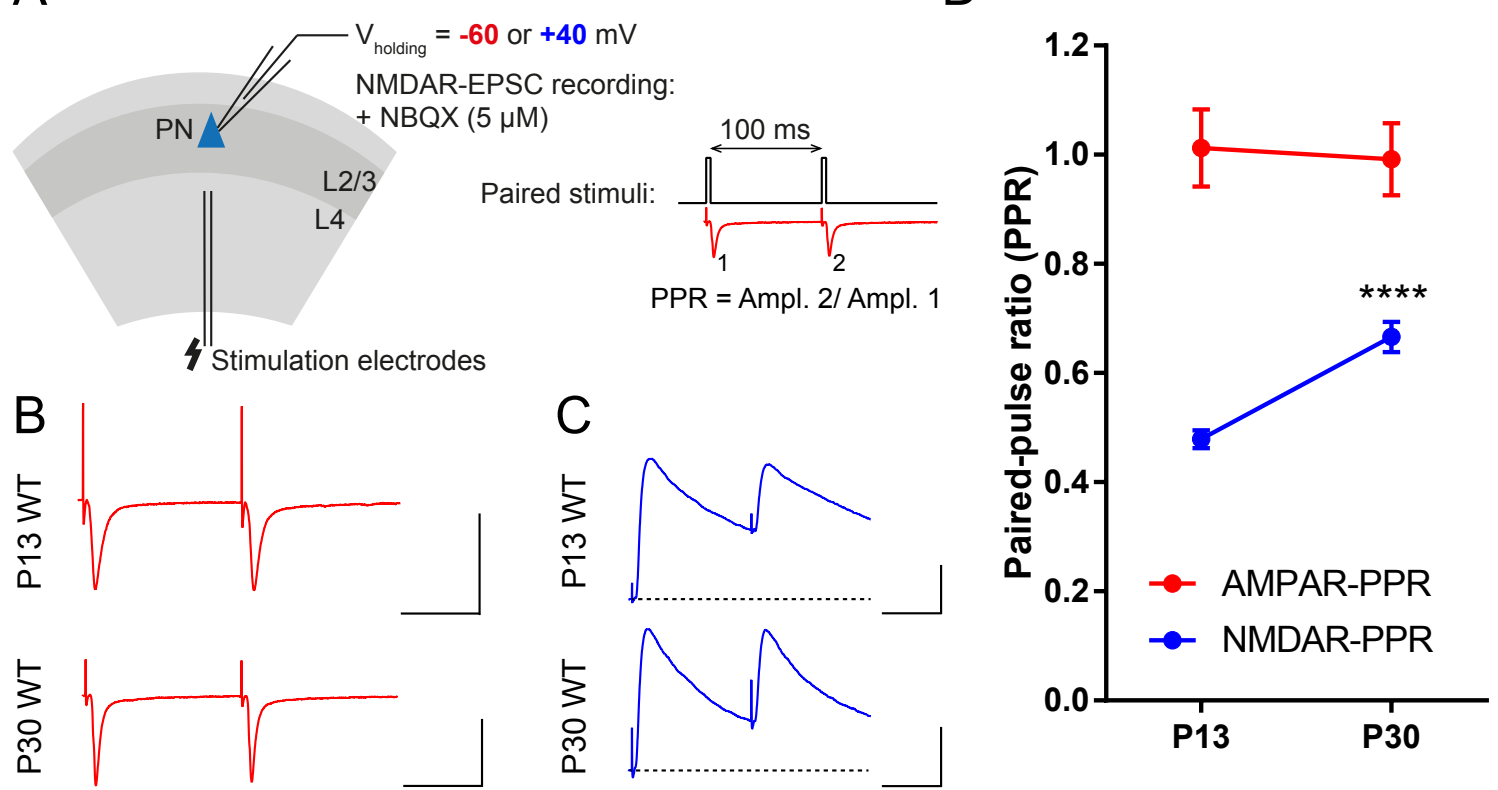

Fig. 3.4. Paired-pulse ratio (PPR) of AMPAR+ synapses was unchanged but PPR of NMDAR+ synapses increased during development.

A: Schematic diagram of the recording configuration. PN: pyramidal neuron. Cells were voltage-clamped at -60 or $+40 \mathrm{mV}$ for the recording of AMPAR-EPSCs or NMDAR-EPSCs respectively. NMDAR-EPSC was recorded in the presence of NBQX $(5 \mu \mathrm{M})$ and the peak response recorded at $+40 \mathrm{mV}$ was considered as NMDAR-EPSC amplitude. Paired stimuli of $100 \mathrm{~ms}$ interval were applied.

B: Example traces of PPR recording at $-60 \mathrm{mV}$ from wild-type (WT) mice at P13 and P30.

C: Example traces of PPR recording at $+40 \mathrm{mV}$ from WT mice at P13 and P30.

D: Means of PPR at different age groups. AMPAR-PPR: PPR of AMPAR+ synapses. NMDAR-PPR: PPR of NMDAR+ synapses.

Age: postnatal day ( \pm 1 day). Scale bars: 200 pA, 50 ms. Error bars: S.E.M. $* * * * \mathrm{P}<0.0001$. Values in table A.1; statistic test results in table A.2.

synapses have higher release probability than AMPAR+ synapses do. The latter scenario is supported by the fact that the increase in NMDAR-PPR coincides with the developmental decrease in silent synapse fraction, as shown by Liu (figure B.1, unpublished). Besides, it has also been shown by other studies that silent synapses have increased release probability (Yanagisawa et al. 2004). Therefore, it is likely that the developmental increase in NMDAR-PPR was primarily caused by the decrease in silent synapse fraction.

\subsubsection{NMDAR blocking by MK-801 slowed down}

Change in PPR is not exclusively a result from a change in release probability. Other approaches measuring release probability with different principles are required. I 
performed another experiment to examines release probability through the MK-801 blocking rate of NMDARs Rosenmund et al., 1993. MK-801 is a non-competitive and irreversible open-channel blocker of NMDARs. It blocks NMDARs only when they are activated. If the release probability is high, more NMDARs will be activated by each stimulus. As a result, more NMDARs will be blocked by MK-801 in a shorter time. Therefore, MK- 801 blocking rate is directly proportional to the release probability. I recorded NMDAR-EPSCs in the presence of $5 \mu \mathrm{M}$ MK-801 and analyzed the decay in NMDAR-EPSC amplitudes (figure 3.5).

The decay in NMDAR-EPSC amplitudes is an exponential decay because the rate of the decay is dependent on the number of unblocked NMDARs. Such decay can be characterized by its half-life $\left(\mathrm{t}_{1 / 2}\right)$ and decay time constant $(\tau)$. Since the half-life of this decay is not constant (figure $3.5 \mathrm{C}$ : decay from $100 \%$ to $50 \%$ is faster than decay from $50 \%$ to $25 \%$ ), it does not follow simple first order kinetics and hence it is not a single exponential decay. Besides, MK-801 blocking is not a multi-step process and does not have branching steps. Therefore, the best fitting decay model is a two-phase exponential decay, with two independent components (fast and slow) decaying in parallel. In this model, there are three variables to fit: $\mathrm{K}_{\text {fast }}, \mathrm{K}_{\text {slow }}$ (rate constants of the fast and slow component, reciprocals of time constants $\tau_{\text {fast }}$ and $\tau_{\text {slow }}$ ) and Percent fast (percentage of fast component) (Equation 2.1).

The existence of two decay components indicates that there are two populations of synapses which have different decay rates and therefore different release probabilities. Synapses with higher release probability should have faster decay, and thus account for the fast component. As shown before, silent synapses likely have higher release probability (Yanagisawa et al. 2004). Therefore, they should account for the fast component of the decay and AMPAR+ synapses for the slow component. The value for Percent $\mathrm{f}_{\text {fast }}$ equals to the percentage of silent synapses.

To obtain the rate constants (i.e. reciprocals of time constants) for each recording, I first fitted the decay with constraining Percent fast $_{\text {to }}$ the percentage of silent synapses (value for P13 wild-type mice from Yuzhang Liu, Pittsburgh (unpublished, see figure B.1); for P30 wild-type from Huang et al. (2015)). To estimate the Percent $_{\text {fast }}$ for each recording, I then fitted the decay with constraining the rate constants of each recording to the previously obtained values. The $\mathrm{R}^{2}$ of fitting

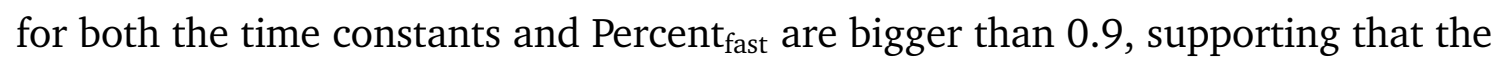
fitting was a good fit. 
There was no significant change in both $\tau_{\text {fast }}$ and $\tau_{\text {slow }}$ (figure 3.5D), suggesting that the release probability for silent and AMPAR + synapses do not change during development. The estimated Percent fast at P30 is lower than at P13 (figure 3.5E), suggesting that the change in NMDAR-PPR detected previously was likely due to the change in silent synapse fraction instead of a change in release probability. It is worth noting that the estimated values for Percent $t_{\text {fast }}$ are similar to the silent synapse fraction assessed by minimal stimulation (figure B.1), providing additional evidence that synapses with high release probability are silent synapses and those with low release probability are AMPAR+ synapses, as also observed in other studies (Yanagisawa et al. 2004). Regardless, the results from PPR and MK-801 blocking rate of NMDARs together suggest that there was likely no change in release probability, and there are likely other mechanisms explaining the discrepancy between the developmental profile of mEPSC frequency and silent synapse fraction.

\subsection{Developmental change in labile synapses was not detected}

Another possible explanation for the the asymmetrical results of mEPSC frequency and silent synapse fraction is the silencing of "labile synapses". Wasling et al. (2012) showed in CA1 region of rat hippocampal slices that, only at early synapses (P7-13) but not at mature synapses (>P26), AMPAR activation by agonists or by electrical stimulation induces AMPAR internalization (and thus AMPAR silencing) while NMDAR signaling is not affected. Their results suggest that some of the early transmitting AMPAR+ synapses are labile, in the sense that they can be transiently converted to AMPAR-silent synapses. If labile synapses also exist in visual cortex, the electrical stimulation employed in the minimal stimulation protocol for silent synapse fraction measurement could possible induce silencing of labile synapses, leading to an overestimation of silent synapse fraction. At the same time, since labile synapses are not silenced during mEPSC recording which does not employ stimulation, they would be detected as AMPAR + synapses. Therefore, developmental maturation of labile synapses into AMPAR + synapses could lead to a decrease in the estimated silent synapse fraction while keeping mEPSC frequency unchanged. This would explain the asymmetrical results between mEPSC frequency and silent synapse fraction. 

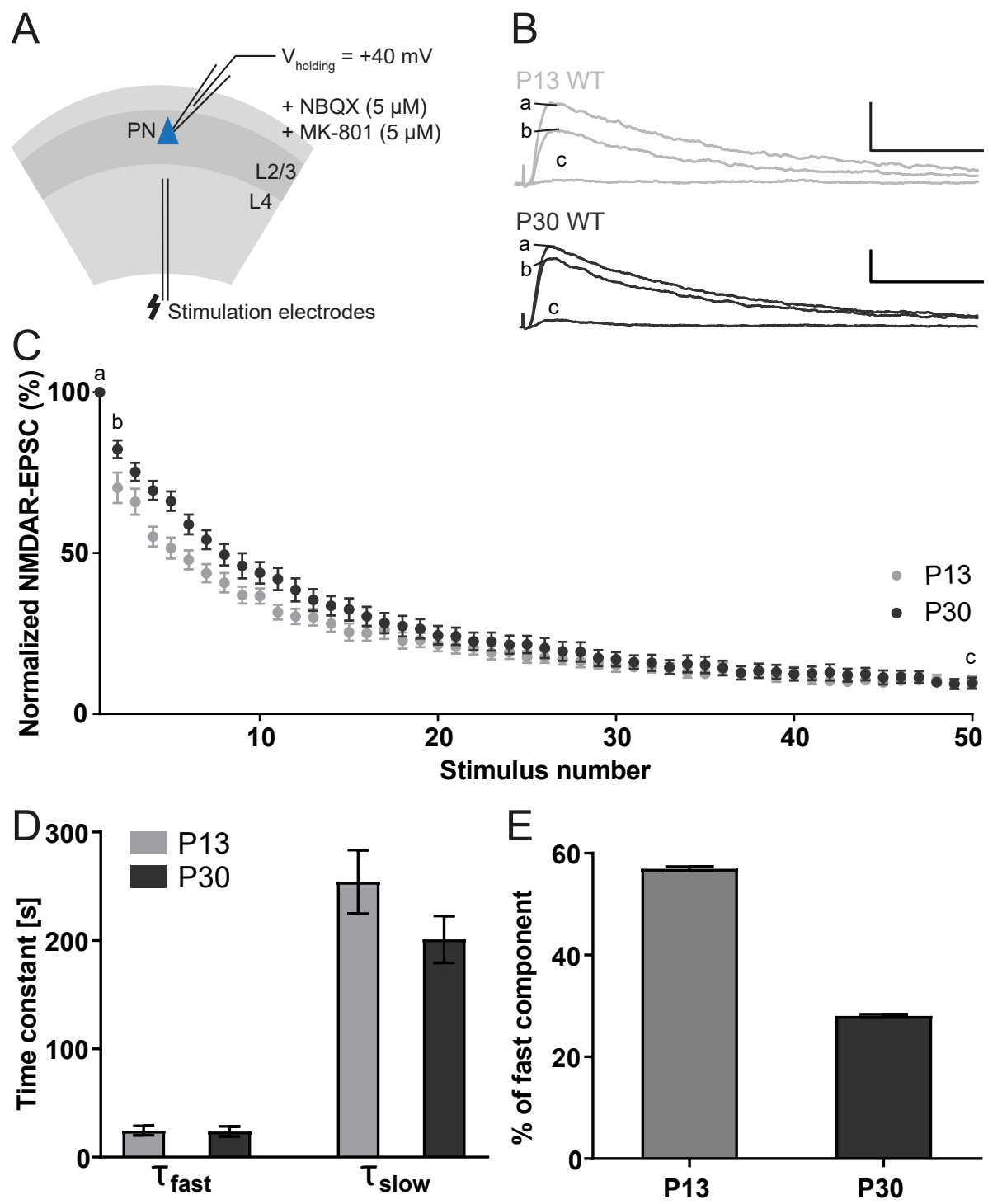

Fig. 3.5. MK-801 blocking rate of NMDARs was slower at P30 compared to P13.

A: Schematic diagram of the recording configuration. PN: pyramidal neuron. Cells were voltage-clamped at $+40 \mathrm{mV}$ and NMDAR-EPSC was recorded in the presence of NBQX $(5 \mu \mathrm{M})$ and MK-801 $(5 \mu \mathrm{M})$. Peak response recorded at +40 $\mathrm{mV}$ was considered as NMDAR-EPSC amplitude.

B: Example traces of NMDAR-EPSC recording from wild-type (WT) mice at P13 and P30. a, b and c are the first, second and the last sweep. Scale bars: 500 pA, $50 \mathrm{~ms}$.

C: Average NMDAR-EPSC amplitude (normalized to the first sweep) at each stimulus number. Time interval between stimuli: $10 \mathrm{~s}$.

D: Time constants of the fast $\left(\tau_{\text {fast }}\right)$ and the slow component $\left(\tau_{\text {slow }}\right)$ of the decay in NMDAR-EPSC amplitude. Average $\mathrm{R}^{2}$ of fitting: 0.9553 for P13; 0.9599 for P30.

E: Estimated percentage of fast component of the decay in NMDAR-EPSC amplitude. Average $\mathrm{R}^{2}$ of fitting: 0.9584 for P13; 0.9609 for P30.

Age: postnatal day ( \pm 1 day). Error bars: S.E.M. ${ }^{*} * * \mathrm{P}<0.001$. Values in table A.1; statistic test results in table A.2 
To detect if developmental change in labile synapses exists in visual cortex, I recorded AMPAR-mEPSCs with a brief application of the AMPAR agonist $\alpha$-amino3-hydroxy-5-methyl-4-isoxazolepropionic acid (AMPA) to silence any potential labile synapses (figure 3.6A and B). If labile synapses exist, one should expect a decrease in mEPSC frequency resulted from the silencing of labile synapses by AMPA application. I observed no significant decrease in frequency at both P11 and P20, suggesting that labile synapses do not exist in the layer 4 to layer $2 / 3$ pyramidal neuron synaptic connection of the visual cortex. Although the decrease in frequency at P30 indicates that there might be labile synapses at P30, higher labile synapse number at P30 would not explain the asymmetrical results of mEPSC frequency and silent synapse fraction, as this would suggest that the silent synapse fraction at P30 was overestimated and that there should be an even larger decrease in the silent synapse fraction. Besides, I compared the change of mEPSC frequency after AMPA application and found no significant difference among P11, P20 and P30, meaning that there was no developmental change in labile synapse number even they do exist. P11 (figure 3.6F). This suggests that change in labile synapses unlikely accounts for the homeostasis of mEPSC frequency after eye opening. Note that mEPSC amplitude remained the same after AMPA application, showing that the recordings were stable. 


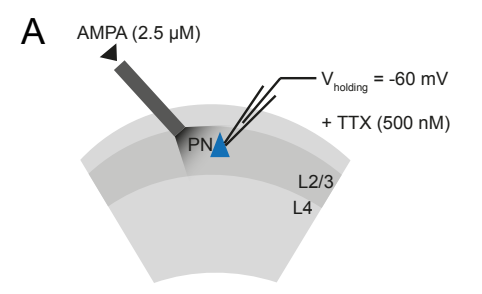

B

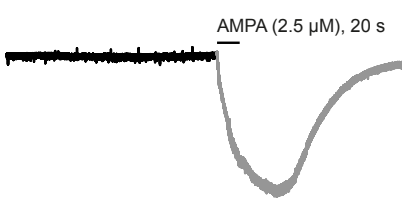

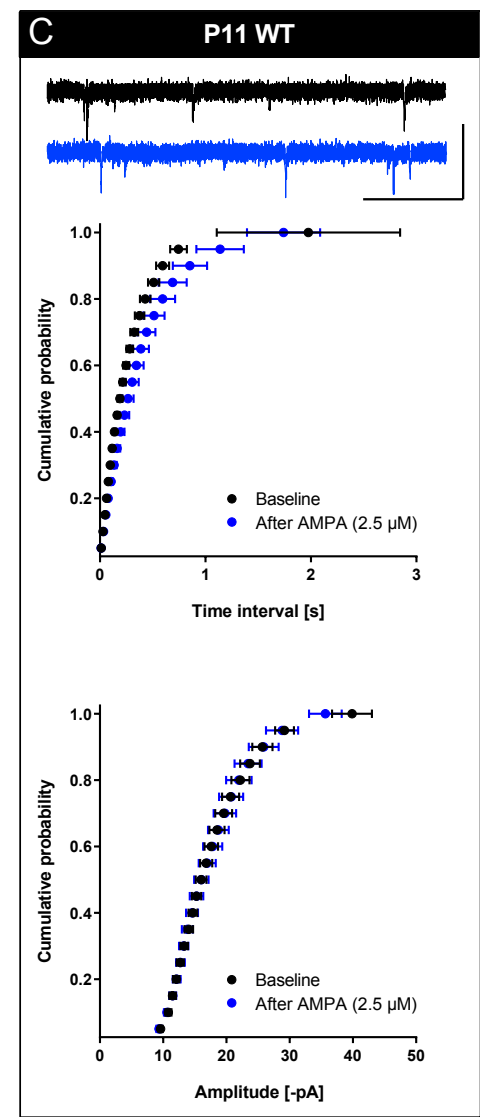

D

P20 WT
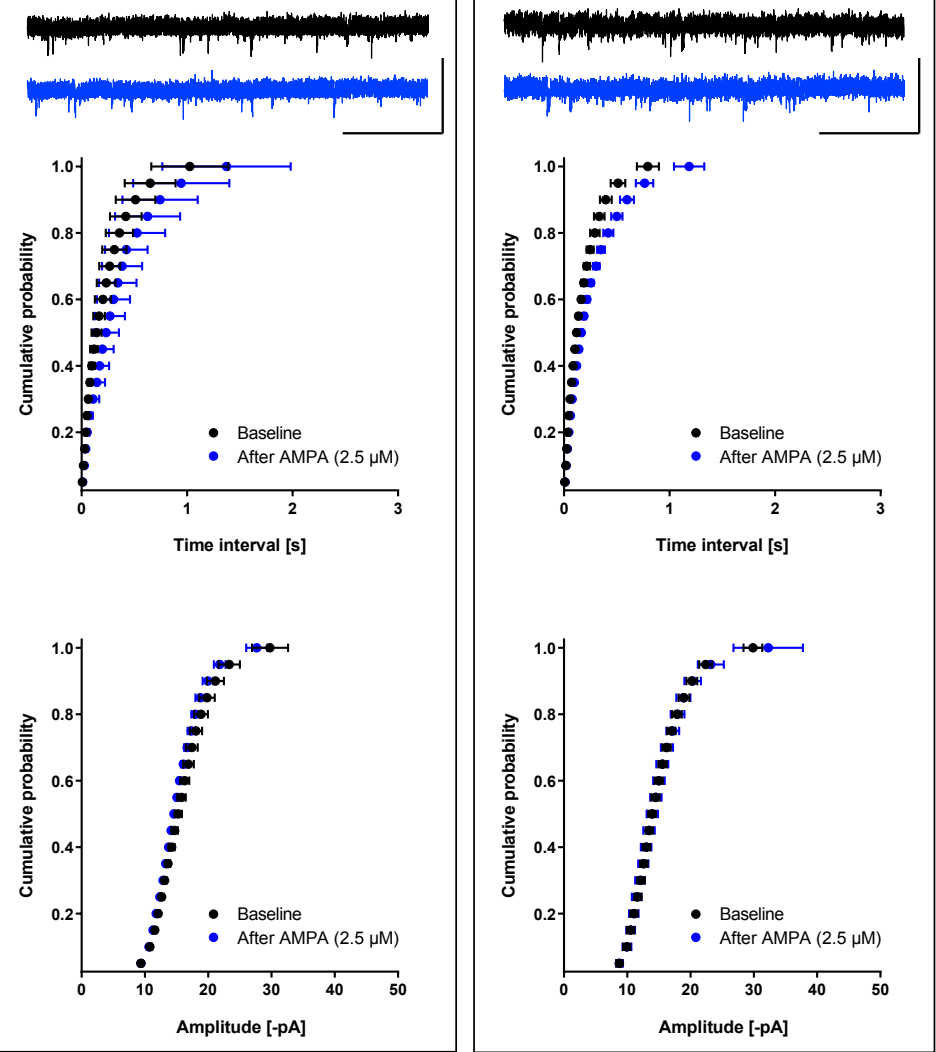

$\mathrm{F}$

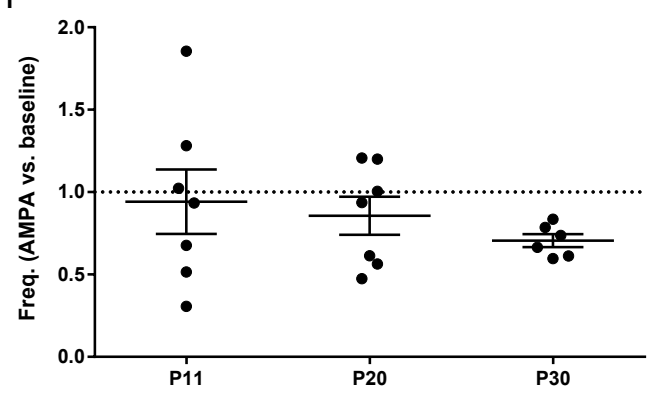

G

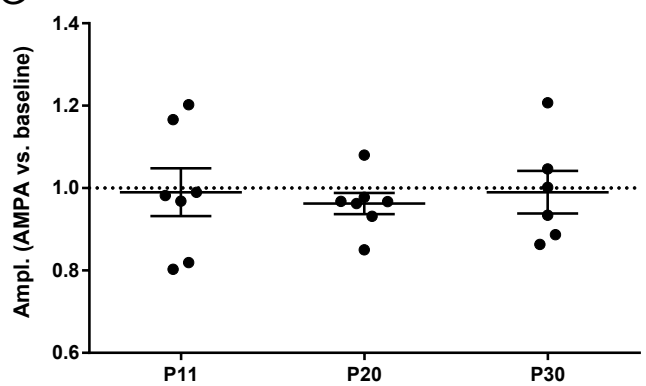

(Figure caption on next page.) 
Fig. 3.6. No difference was observed in AMPA-induced silencing of AMPAR+ synapses at different ages. (Figure on previous page.)

A: Schematic diagram of the recording configuration. PN: pyramidal neuron. Cells were voltage-clamped at $-60 \mathrm{mV}$ and $\mathrm{mEPSCs}$ of AMPAR + synapses (AMPARmEPSCs) were recorded in the presence of tetrodotoxin (TTX, $500 \mathrm{nM}$ ). AMPA $(2.5 \mu \mathrm{M})$ was applied via local perfusion.

B: Illustration of the experimental design. mEPSCs were recorded before AMPA application as the baseline (black). AMPA was then applied for 20 s. mEPSCs 6.5 min after AMPA application (blue) were used for analysis. Scale bars: 200 pA, $200 \mathrm{~s}$.

C-E: Top: example traces of mEPSC recording before (black) or after AMPA application (blue) from wild-type (WT) mice at different ages. Scale bars: $50 \mathrm{pA}$, $500 \mathrm{~ms}$. Middle and bottom: cumulative probability of mEPSC time interval and amplitude. Bin size: 20 events.

F and G: Frequency (F) and amplitude (G) after AMPA application (normalized to recording before AMPA application). Each dot represents one cell. Horizontal line of each column represents the mean.

Age: postnatal day ( \pm 1 day). Error bars: S.E.M. Values in table A.1; statistic test results in table A.2.

\subsection{Novel role of AMPAR desensitization during development}

\subsubsection{AMPAR desensitization blocker TCM increased mEPSC frequency}

Besides AMPAR internalization in labile synapses, AMPAR + synapses could be transiently silenced due to AMPAR desensitization. Some synapses might contain AMPARs which are in desensitized state for a long period of time, or they are more prone to become desensitized. This could compensate the increase in mEPSC frequency due to silent synapse maturation, providing another possible mechanism for homeostasis of AMPAR-mEPSC frequency. To test if there is any change in the number of desensitized AMPARs during development, I recorded AMPAR-mEPSCs before and during the bath application of a desensitization blocker trichlormethiazide (TCM) (figure 3.7A and B). TCM's effects on mEPSC properties at P13 and P30 was compared. I used PSD-95 KO mice at P30 as a negative control because of their high silent synapse fraction.

It has been reported by several studies that cyclothiazide (CTZ), another chemically related desensitization blocker, increases mEPC frequency by more than two folds 
to nearly six folds (Deng and Chen, 2003, Diamond and Jahr, 1995, Ishikawa and Takahashi, 2001; Mennerick and Zorumski, 1995, Montgomery et al., 2001; Yamada and Tang, 1993. In this study, TCM increased AMPAR-mEPSC frequency by more than three folds in WT at P13 and P30, as well as in PSD-95 KO at P30 (figure 3.7F). The mEPSC amplitude (figure 3.7G) and decay time (figure 3.7 H) were also increased, confirming that AMPAR desensitization was blocked by TCM. Although there was no significant difference in TCM's effect between P13 and P30 to account for the homeostasis of mEPSC frequency, the sample size was not big enough to provide statistic power to draw a conclusion with such large variation in fold change (ranging from less than two folds to nearly 8 folds). More recordings are needed for confirmation.

As mentioned earlier, an increase in mEPSC frequency may reflect an increase in presynaptic vesicle fusion or an increase in synapse number. While AMPAR desensitization blockers act postsynaptically, nonspecific presynaptic effects of CTZ have also been suggested by several studies Barnes-Davies and Forsythe, 1995; Bellingham and Walmsley, 1999, Diamond and Jahr, 1995, Ishikawa and Takahashi, 2001). However, such effects have not been observed with TCM. To test if TCM alters presynaptic vesicle release, I looked at the MK-801 blocking rate of NMDARs in the presence of TCM in the following section. 
A

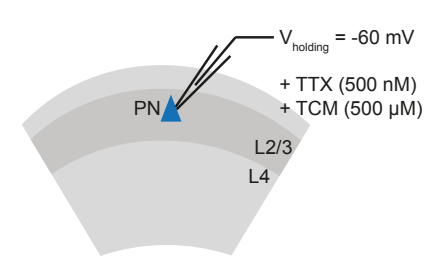

\section{C}

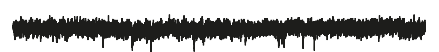

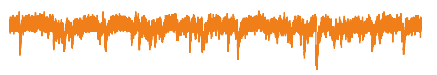
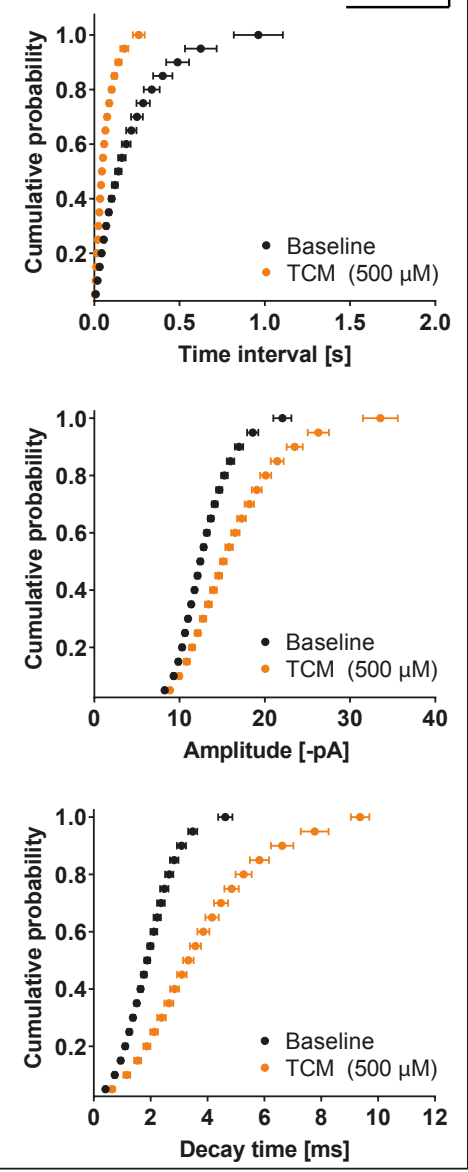

$\mathrm{F}$

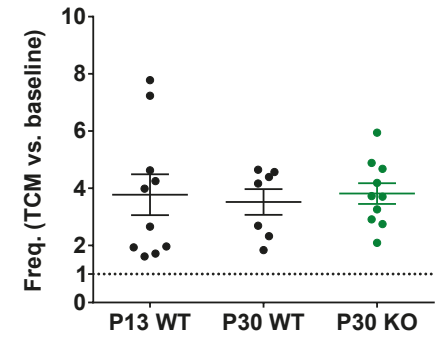

B

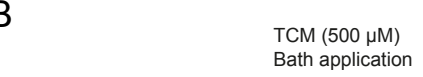

D

P30 WT
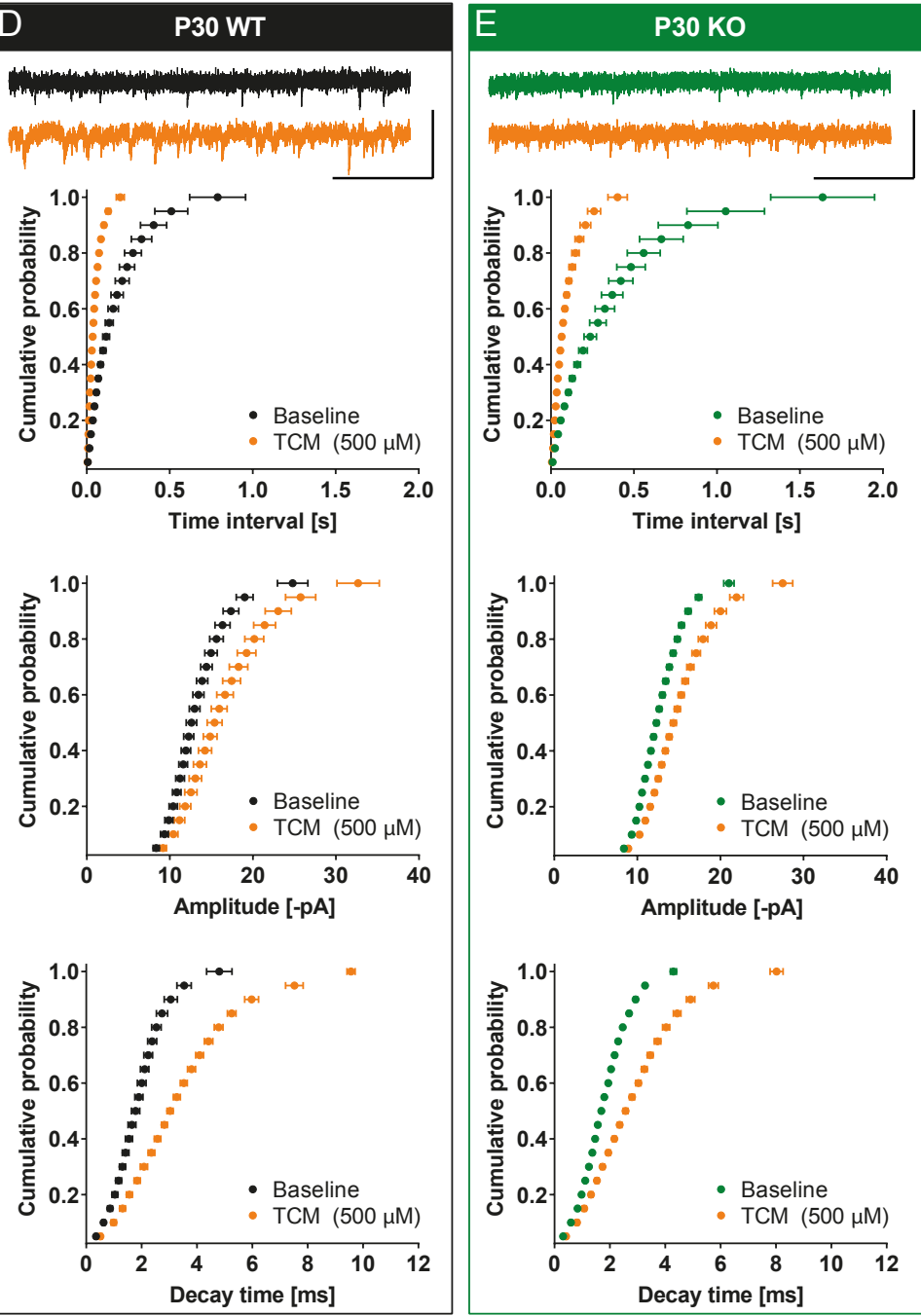

G

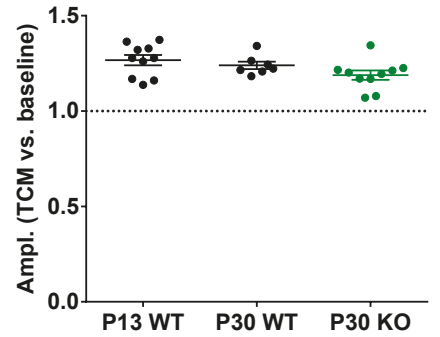

$\mathrm{H}$

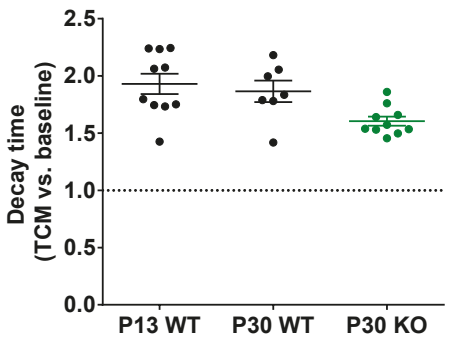

(Figure caption on next page.) 
Fig. 3.7. AMPAR desensitization blocker TCM increased mEPSC frequency in WT at $\mathrm{P} 13, \mathrm{P} 30$ and in PSD-95 $\mathrm{KO}$ at P30. (Figure on previous page.)

A: Schematic diagram of the recording configuration. PN: pyramidal neuron. Cells were voltage-clamped at $-60 \mathrm{mV}$ and mEPSCs of AMPAR + synapses (AMPARmEPSCs) were recorded in the presence of tetrodotoxin (TTX, $500 \mathrm{nM}$ ). TCM $(500 \mu \mathrm{M})$ was bath applied.

B: Illustration of the experimental design. mEPSCs were recorded before TCM application as the baseline (black). TCM was then applied until the end of the recording. mEPSCs $2 \mathrm{~min}$ after the start of TCM application (orange) were used for analysis. Scale bars: $50 \mathrm{pA}, 60 \mathrm{~s}$.

C-E: Top: example traces of mEPSC recording before (black for WT; green for PSD-95 KO) or during TCM application (orange) from wild-type (WT) mice at P13 and P30; from PSD-95 knock-out (KO) mice at P30. Scale bars: 50 pA, 500 ms. Plots below example traces to bottom of panel: cumulative probability of mEPSC time interval, amplitude and decay time. Bin size: 20 events.

F-H: Frequency (F), amplitude (G) and decay time (H) during TCM application (normalized to recording before TCM application). Each dot represents one cell. Horizontal line of each column represents the mean.

Age: postnatal day ( \pm 1 day). Error bars: S.E.M. Values in table A.1 statistic test results in table A.2.

\subsubsection{TCM did not change the MK-801 blocking rate of NMDARs}

To check if TCM affects presynaptic neurotransmitter release, I recorded NMDAREPSCs with $5 \mu \mathrm{M}$ MK-801 and compared the decay in NMDAR-EPSC amplitude in the absence and the presence of TCM. I found no difference in both $\tau_{\text {fast }}$ and $\tau_{\text {slow }}$ of MK-801 blocking rate with and without TCM (figure 3.8D). There was also no difference in Percent ${ }_{\text {fast }}$ (figure 3.8E). This indicates that TCM did not affect the release probability of the synapses, and that the increase in mEPSC frequency was not due to an increase in release probability.

\subsubsection{TCM only slightly increased evoked EPSC amplitude}

As shown previously, TCM increased mEPSC frequency by more than three fold without changing release probability. It is possible that the increase in frequency by TCM was due to an increase in the number of AMPAR + transmission sites. These initially non-transmitting AMPAR + transmission sites, if they do exist, are apparently neither detected as AMPAR + synapses nor silent synapses by minimal 
A

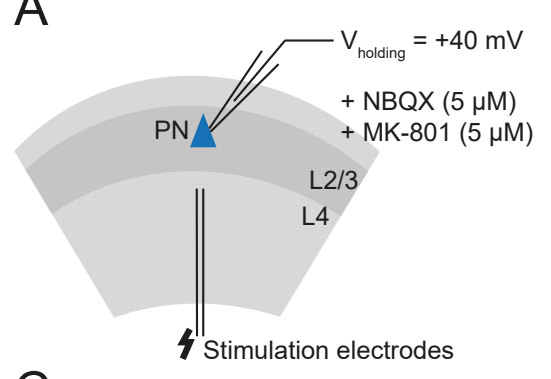

C

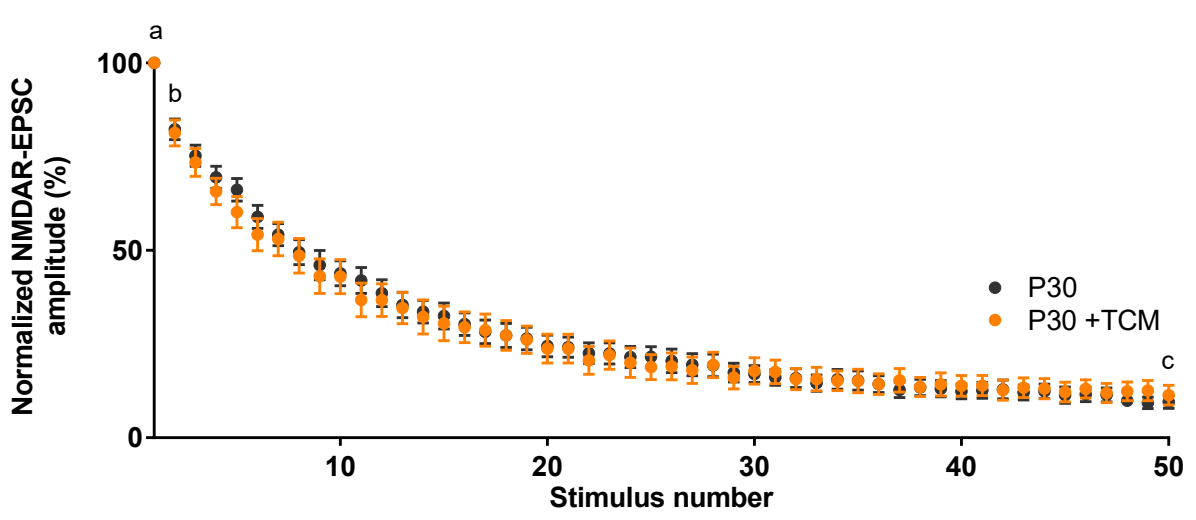

B

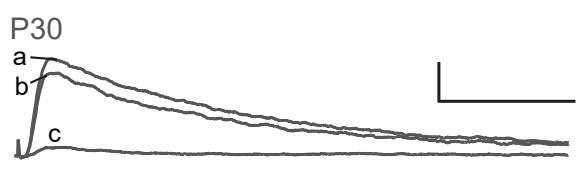

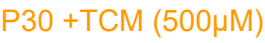

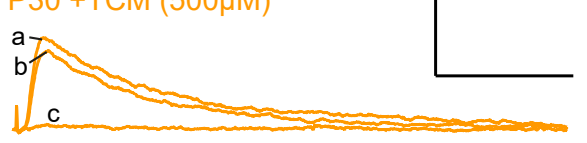

$E$
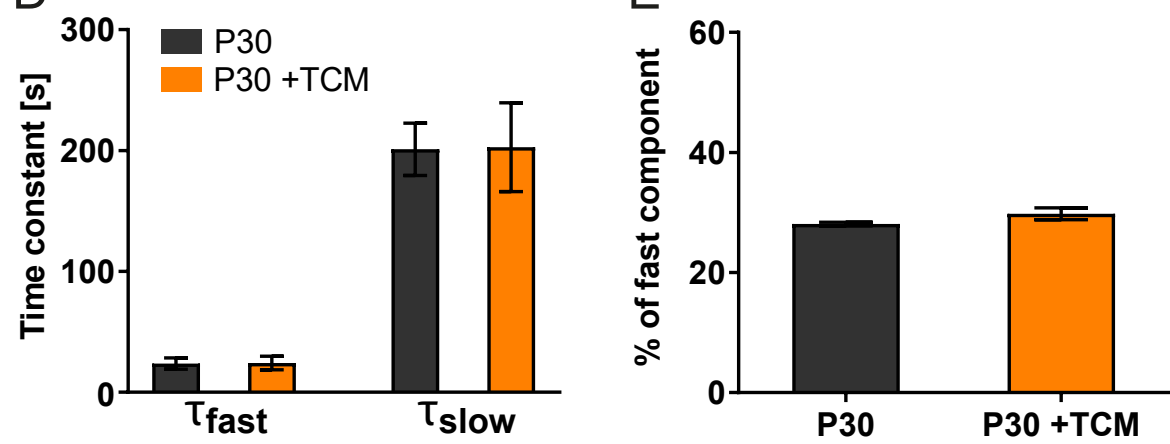

Fig. 3.8. MK-801 blocking rate of NMDARs was not affected by desensitization blocker TCM.

A: Schematic diagram of the recording configuration. PN: pyramidal neuron. Cells were voltage-clamped at $+40 \mathrm{mV}$ and NMDAR-EPSC was recorded in the presence of NBQX $(5 \mu \mathrm{M})$ and MK-801 $(5 \mu \mathrm{M})$, with or without TCM $(500 \mu \mathrm{M})$. Peak response recorded at $+40 \mathrm{mV}$ was considered as NMDAR-EPSC amplitude. B: Example traces of NMDAR-EPSC recording with and without TCM from wildtype (WT) mice at P30. a, b and c are the first, second and the last sweep. Scale bars: $500 \mathrm{pA}, 50 \mathrm{~ms}$.

C: Average NMDAR-EPSC amplitude (normalized to the first sweep) at each stimulus number. Time interval between stimuli: $10 \mathrm{~s}$.

D: Time constants of the fast $\left(\tau_{\text {fast }}\right)$ and the slow component $\left(\tau_{\text {slow }}\right)$ of the decay in NMDAR-EPSC amplitude. Average $\mathrm{R}^{2}$ of fitting: 0.9599 for P30; 0.9466 for P30+TCM.

E: Estimated percentage of fast component of the decay in NMDAR-EPSC amplitude. Average $\mathrm{R}^{2}$ of fitting: 0.9609 for P30; 0.9531 for P30+TCM.

Age: postnatal day ( \pm 1 day). Error bars: S.E.M. Values in table A.1; statistic test results in table A.2. 
stimulation, otherwise their potentially huge number would greatly shift the silent synapse fraction. Since minimal stimulation relies on evoked EPSC responses, this raises a question: do these sites transmit evoked responses at all? To test this, I recorded evoked AMPAR-EPSCs before and during bath application of TCM (figure 3.9A and D). Given that TCM increased mEPSC frequency (no matter the increase was due to larger synapse number or higher release probability), an huge increase in evoked EPSC amplitude was expected. However, I found that the increase in EPSC amplitude by TCM was only subtle compared to the more than three-fold increase in mEPSC frequency (figure 3.9B). Similar to the slight increase in mEPSC amplitude, increase in EPSC amplitude could be due to TCM's effect on the existing AMPAR + synapses. This suggests that transmission sites recruited by TCM probably do not have evoked synaptic transmission. Nevertheless, the sample size here is small $(\mathrm{N}=4)$ and therefore the variance is large. More recordings will be performed to confirm the result.

\subsection{Developmental increase in total surface AMPAR number}

Besides the asymmetrical results of mEPSC frequency and silent synapse fraction, another interesting observation from the developmental change in mEPSC frequency was that PSD-95 KO mice had similar developmental change in mEPSC frequency despite mEPSC frequency was reduced. Moreover, mEPSC amplitude was only slightly affected by PSD-95 deletion and the developmental change was not even affected by PSD-95 deletion. We wondered whether PSD-95 was necessary in AMPAR trafficking during development. Since interpretation of mEPSC results could be complicated by presynaptic factors such as release probability, I assessed the developmental change in total surface AMPAR number with recording independent of presynaptic neurotransmitter release: the current response to local application of exogenous AMPA. I performed whole-cell voltage clamp recording in both WT and PSD-95 KO mice (figure 3.10A). I found that AMPA response increased during development from P11 to P30 in both WT and PSD-95 KO mice (figure 3.10B). In addition, AMPA response in PSD-95 mice was the same as in WT mice at P30 though they had slightly lower AMPA response at earlier stages. This suggests that PSD-95 is not strictly required for the surface expression of AMPARs. In contrast, the prominent effect of PSD-95 deletion on mEPSC frequency suggests that PSD-95's role in AMPAR-mediated transmission mainly lies in the regulation 

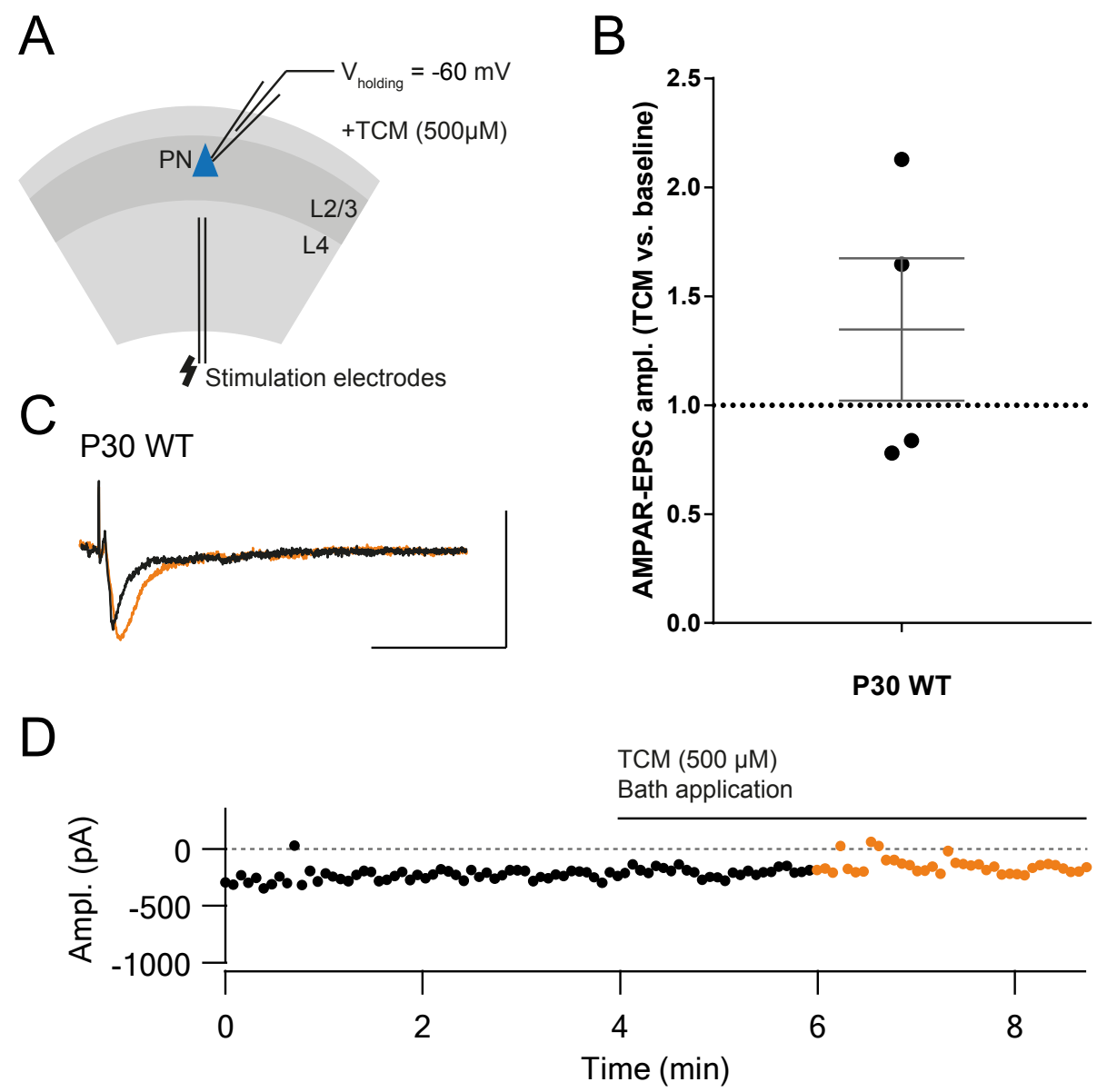

Fig. 3.9. AMPAR desensitization blocker TCM only had a subtle effect on evoked EPSC amplitude in wild-type mice at P30.

A: Schematic diagram of the recording configuration. PN: pyramidal neuron. Cells were voltage-clamped at $-60 \mathrm{mV}$ and AMPAR-EPSC was recorded with or without bath application of TCM $(500 \mu \mathrm{M})$.

B: Change in AMPAR-EPSC amplitude during TCM application. Each dot represents one cell. Horizontal bar represents the mean.

C: Example traces of AMPAR-EPSC recording with (orange) and without TCM (black) from wild-type (WT) mice at P30.

D: Example of one recording. AMPAR-EPSCs were recorded before TCM application as a reference (black dots). TCM was then applied until the end of the recording. AMPAR-EPSCs 2 min after the start of TCM application (orange dots) were used for analysis.

Age: postnatal day ( \pm 1 day). Error bars: S.E.M. Values in table A.1. Scale bar: $200 \mathrm{pA}, 50 \mathrm{~ms}$. 
of AMPAR trafficking to the synapses rather than the exocytosis of AMPARs to the surface. 


\section{A}

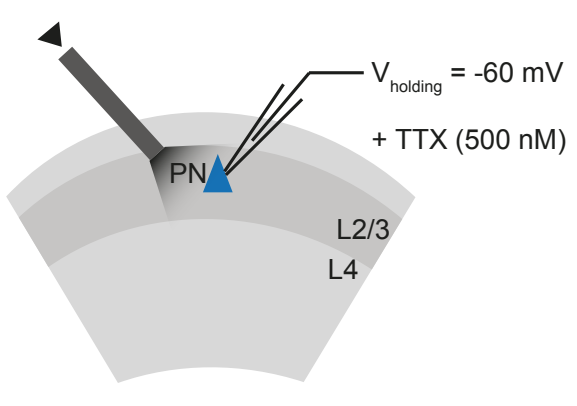

C
B

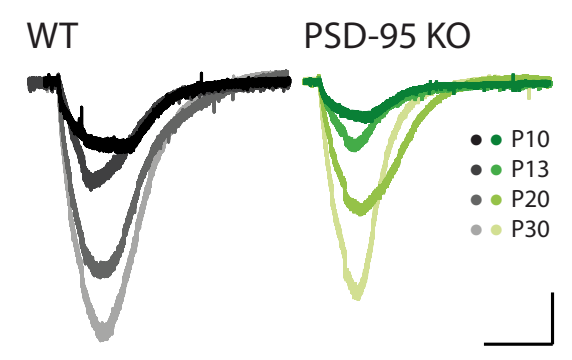

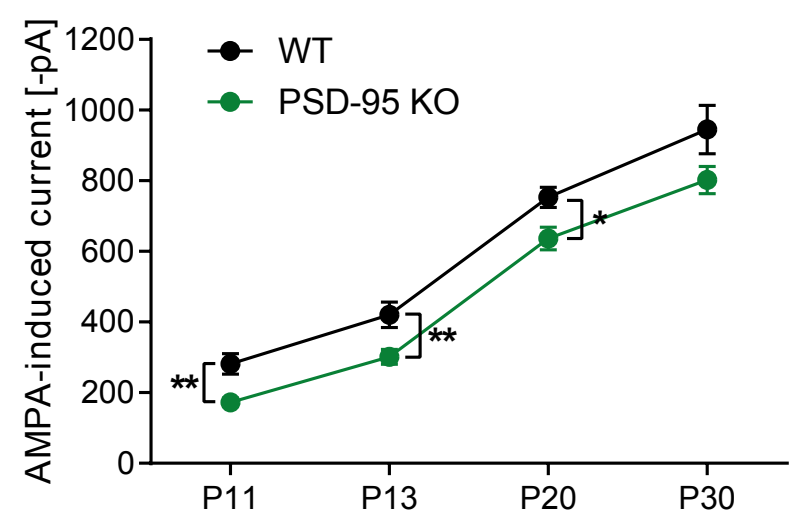

Fig. 3.10. Developmental increase in AMPA response was observed in wild-type and PSD-95 KO mice.

A: Schematic diagram of the recording configuration. PN: pyramidal neuron. Cells were voltage-clamped at $-60 \mathrm{mV}$ and current response to AMPAR agonist AMPA was recorded in the presence of tetrodotoxin (TTX, $500 \mathrm{nM}$ ). AMPA (2.5 $\mu \mathrm{M})$ was applied via local perfusion for $20 \mathrm{~s}$.

B: Example traces of AMPA response at different age groups of wild-type (WT) and PSD-95 KO mice.

C: Summary of AMPA response amplitude at different age groups of wild-type (WT) and PSD-95 KO mice. WT vs. PSD-95 KO (Welch's t-test): $\mathrm{P}=0.0034$ for $\mathrm{P} 11 ; \mathrm{P}=0.0145$ for $\mathrm{P} 13 ; \mathrm{P}=0.0134$ for $\mathrm{P} 20 ; \mathrm{P}=0.0816$ for $\mathrm{P} 30$.

Age: postnatal day ( \pm 1 day). Error bars: S.E.M. ${ }^{*} p<0.05 * * p<0.01$. Scale bar: 200 pA, $50 \mathrm{~s}$. Values in tableA.1; statistic test results in table A.2. 



\section{Discussion}

\section{1 circuit refinement parallel to silent synapse maturation}

Circuit refinement during development involves rewiring through elimination and generation of synapses. Maturation of silent synapses to AMPAR + synapses should mediate at least part of this process. As shown by Huang et al. (2015), the silent synapse fraction decreases during development in visual cortex. If circuit refinement involves solely silent synapse maturation, this would predict an increase in the number of AMPAR + synaptic connections. However, I did not observe a corresponding increase in mEPSC frequency throughout development: mEPSC frequency increased only before eye opening but remained unchanged afterwards (figure 3.1). Indeed, our follow-up experiment found that the decrease in silent synapse fraction starts only after eye opening (figure B.1, unpublished). This asymmetrical result of mEPSC frequency and silent synapse fraction hints that circuit refinement involves more than just the conversion of silent synapses to AMPAR + synapses.

\subsubsection{Eye opening (P13): critical point in synapse development}

Eye opening is a critical point in visual development. Animals start to receive patterned visual input which is essential for activity-dependent plasticity such as the binocular matching of orientation preference of binocular vision. This particularly important developmental stage is expected to have significant influence of synapse maturation. I observed about a doubled increase in AMPAR-mEPSC frequency upon eye opening (P13) in both WT and PSD-95 KO mice (figure 3.1), consistent with previous findings that mEPSC frequency increases around eye opening (Desai et al. 2002). Since AMPAR-PPR did not change upon eye opening (figure 3.3), the increase in mEPSC frequency was likely mediated by an increase in the AMPAR + synapse number instead of an increase in presynaptic release. Nevertheless, I cannot exclude the possibility that there could be an actually change 
in PPR due to a change in release probability, just that it is compensated by some change in postsynaptic properties such as AMPAR desensitization at young age. Other methods to assess presynaptic release are needed to confirm the results. For example, presynaptic release can be assessed by measuring the MK-801 blocking rate of NMDAR response, which uses NMDARs as another postsynaptic reporter of presynaptic properties.

An increase in AMPAR + synapse number would contradict the change in silent synapse fraction because a corresponding change in silent synapse fraction upon eye opening was absent (figure B.1), although the unchanged ratio could be explained by an equal increase in both silent and AMPAR + synapse number. This explanation seems to be supported by the increase in total synapse number during development as suggested by an increase in spine density (figure B.2, unpublished).

Another significant change upon eye opening was the drop in AMPAR-mEPSC amplitude (figure 3.1), consistent with Desai et al. (2002)'s study. This suggests that there was a decrease in the quantal size at AMPAR+ synapses, likely due to a decrease in AMPAR number at single synapses. During development, a decrease in quantal size means that a wider dynamic range for postsynaptic response is possible. Considering the large amount of synapses each neuron has, a small decrease in quantal size would greatly increase the computational power of a neuron. This potentially enhances the capacity of the neuron to process information from more inputs. After the initial drop, the quantal size was maintained at least near the end of critical period (P30). This could mean that smaller quantal size is favored for the fine tuning of different modalities of visual input during the critical period, when a neuron presumably receives more inputs after eye opening.

In addition, changes in quantal size has been proposed as a mechanism for homeostatic synaptic scaling, which maintains the relative strength of different inputs by scaling all synaptic inputs globally (Turrigiano, 1999, 2008). Such mechanism has been observed both in vitro (O’Brien et al. 1998; Turrigiano et al., 1998) and in vivo (Desai et al. 2002). In this study, the decrease in mEPSC amplitude upon eye opening was in parallel with the increase in frequency, and both remained unchanged afterwards. Therefore, the decrease in quantal size is could be the neuron's response to the increasing mEPSC frequency, though it is also possible that this was a response to the start of visual inputs. Several physiological functions of synaptic scaling have been suggested, such as maintaining network stability and retaining information from different inputs during development or learning (Turrigiano, 2008). 
Major changes in mEPSC properties upon eye opening revealed eye opening as a critical point in synapse development of visual cortex. These changes could be activity-dependent, i.e. relying on the visual input upon eye opening. To test the activity requirement for these changes, mEPSCs can be recorded in dark-reared animals and check if the same changes still happen. Nevertheless, it should be noted that the same trend of developmental changes in mEPSC properties still occurred when PSD-95 was deleted. Since PSD-95 deletion and dark rearing of mice from birth have the same effect on preventing silent synapse maturation, such effect could arise from the same intervention on synapse development. This means that, similar to PSD-95 deletion, dark rearing might not influence the trend of developmental changes in mEPSC properties though it might change the size of the changes. Alternatively, these changes could be an intrinsic property of a neuron to prepare itself for the increased neural activity after eye opening.

Deletion of PSD-95 could only affect the size of the changes (as shown by the decreased mEPSC frequency at P13), but not when the changes occur. It is possible that the size of the changes and the time point when the changes take place are differentially regulated. Lack of changes after eye opening in both WT and PSD-95 KO mice suggested that eye opening is a critical time point when the level of neural activity is set.

\subsubsection{Homeostasis of AMPAR-mEPSC frequency after eye opening}

As discussed earlier, the mEPSC frequency was set at the time point of eye opening and was maintained after eye opening (i.e. a homeostasis of frequency), at least till P30 near the end of critical period (figure 3.1). Similar results have also been observed in other studies (Desai et al., 2002, Han et al., 2017). Such observation did not agree with the developmental decrease in silent synapse fraction, which predicts an increase in AMPAR + synapse number and thus an increase in mEPSC frequency. What could be the mechanism for this homeostasis of mEPSC frequency while there is a concurrent decrease in silent synapse fraction? 


\section{Mechanism for homeostasis of mEPSC frequency}

One possible mechanism one may think of is that there might be a decrease in release probability which reduces mEPSC frequency and compensates for the increase in frequency caused by increased number of AMPAR + synapses. However, as shown by the identical AMPAR-PPR at P13 and P30 (figure 3.3), compensatory change in release probability was unlikely the mechanism for this homeostasis. One might argue that AMPAR-PPR could be influenced by AMPAR desensitization. Desensitization could possibly reduce the EPSC amplitude of the second response, resulting in a reduced PPR. If AMPARs experience stronger desensitization at P30, the reduction in PPR due to desensitization would be bigger at P30 and this would cancel the increase in PPR that one would expect for a decrease in release probability. However, it has been shown that AMPAR desensitization actually becomes weaker during development (Joshi et al., 2004). If there was AMPAR desensitization, one should see an increase in PPR instead. It was unlikely that AMPAR-PPR in this study was influenced by AMPAR desensitization, masking any changes in release probability. Besides, the results from MK-801 blocking rate of NMDARs showed that there was no change in the time constants during development, indicating that there was no change in the release probability of AMPAR+ synapses and silent synapses (see Section 4.2).

Another possible mechanism for the homeostasis of mEPSC frequency involves maturation of "labile synapses". As briefly discussed in Section 3.3, labile synapses are functional AMPAR + synapses which can be silenced by agonists or electrical stimulation (Wasling et al. 2012). They could be silenced during recording with minimal stimulation used for the estimation of silent synapse fraction. If labile synapses mature into stable AMPAR + synapses during development, labile synapse number would decrease and thus silent synapse fraction would appear to be decreased, without any change in mEPSC frequency. Using AMPA to induce labile synapse silencing as in the study done by Wasling et al. (2012), I did not observe decrease in AMPAR-mEPSC frequency after AMPA application at P11 and P20 in visual cortex (figure 3.6). This suggests that synapse silencing by AMPA did not happen, i.e. there was no labile synapses in visual cortex at these developmental stages. Note that there was a slight decrease in mEPSC frequency at P30, suggesting that visual cortex may have different developmental profile in terms of labile synapses, as these synapses exist only at early development stages in hippocampus Wasling et al. 2012). Regardless, by comparing the mEPSC frequency change at different age groups, I did not find any developmental increase in the change, meaning that there was no developmental decrease in labile synapse number. Therefore, 
silencing of labile synapse was unlikely the mechanism for the homeostasis of mEPSC frequency after eye opening.

Apart from labile synapses, synapses could also be transiently silenced by AMPAR desensitization. Transient synapse silencing by AMPAR desensitization could serve as a reversible way to control AMPAR + synapse number during development, and thus facilitates the homeostasis of mEPSC frequency. By adding the desensitization blocker TCM, the mEPSC frequency at both P13 and P30 was increased by more than three folds while the mEPSC amplitude was only slight increased (figure 3.7). Increase in mEPSC frequency suggests that TCM unsilenced AMPAR transmission via additional transmission sites (see Section 4.3 for further discussion). In this study, a similar increase in mEPSC frequency at the two age groups was observed though the sample size at P13 was small and the variability was big. With larger sample size, my colleague found that there was indeed a higher increase in mEPSC frequency at P30 (figure B.3, unpublished). This means that homeostasis of mEPSC frequency may be achieved through silencing of AMPAR transmission by AMPAR desensitization, although verification of TCM's actions on these AMPARs is still needed (see Section 4.3 for further discussion).

Homeostasis of mEPSC frequency could also be achieved by pruning of synapses. To refine and optimize neural circuits, it is possible that unused synapses are pruned while new synapses are formed. If there is pruning of old or unused AMPAR+ synapses and formation new AMPAR+ synapses through silent synapse maturation, there would be a decrease in silent synapse fraction while mEPSC frequency (i.e. AMPAR + synapse number) is maintained. Alternatively, there could be simply pruning of silent synapses which would only decrease the silent synapse fraction without changing the mEPSC frequency, although production of excessive silent synapses and elimination of them might not seem efficient intuitively. Finally, it is possible that there is pruning and forming of both silent and AMPAR + synapses in a way that gain and loss of AMPAR + synapses are balanced. All the above hypotheses indicate a decrease in total synapse number. This is in odd with the developmental increase in spine density (figure B.2), although change in spine density does not necessarily represent change in synapse density. In addition, a limitation of the spine density measurement employed here is that all spines were stained with the same dye. Distinguishing between excitatory synapses onto pyramidal neurons and onto interneurons, as well as between silent and AMPAR + synapses, was impossible. Alternative methods, such as simultaneous staining of presynaptic marker (e.g. an active zone protein), postsynaptic marker (e.g. a PSD protein) and 
glutamate receptor (e.g. NMDAR or AMPAR) can be performed to identify silent and/or AMPAR+ excitatory synapses.

\section{Implication from homeostasis of mEPSC frequency}

From the above discussion, homeostasis of mEPSC frequency is most probably achieved by synapse silencing (in the form of "idle site", see Section 4.3) or synapse pruning, though the two mechanisms are not mutually exclusive. Homeostasis of mEPSC frequency may reflect not only a maintenance of overall level of neural activity during development, but also the circuit refinement during development: formation of new synapses in parallel of removal of old or unused synapses. The outcome of the refinement is likely an optimized circuit with same number of AMPAR + synapses, i.e. a more efficient circuit. This may suggest a general developmental strategy that neural circuit is formed first without too much precision while refining of the circuit comes later in the development, instead of building a precise circuit on the way during development. The biggest advantage of this strategy is probably a better adaptation of the circuit to the developmental conditions.

\subsection{Developmental decrease in release probability}

In this study, release probability of AMPAR + synapses was unchanged from P13 to P30 as indicated by the unchanged AMPAR-PPR (figure 3.3). Again, developmental increase in AMPAR-PPR could be canceled out by stronger AMPAR desensitization at P30, though this is unlikely the case as discussed in Section 4.1.2.

\subsubsection{Silent synapses had higher release probability}

\section{Evidences for higher release probability at silent synapses}

NMDAR-PPR was lower than the AMPAR-PPR at both P13 and P30 (figure 3.4). Since both silent and AMPAR + synapses contain NMDARs, difference in NMDARPPR and AMPAR-PPR suggests that silent synapses and AMPAR+ synapses have different release probability. Lower value of NMDAR-PPR was likely due to the 
higher release probability of silent synapses. Moreover, there was an developmental decrease in NMDAR-PPR from P13 to P30. Given that the silent synapse fraction is lower at P30 than P13 (figure B.1), it is likely that the decrease in NMDAR-PPR was caused by the decrease in the fraction of silent synapses which likely have higher release probability. Nevertheless, PPR could also be influenced by receptor desensitization, recovery and exchange during the two stimuli, although the other experiment using MK-801 to assess release probability also points to a higher release probability in silent synapses.

Decrease in MK-801 blocking rate of NMDARs during development (figure 3.5) suggests that release probability is lower at $\mathrm{P} 30$, consistent with the results from NMDAR-PPR. The decay in NMDAR-EPSC amplitude consists of two components: fast and slow component. As mentioned in Section 3.2.3, the fast and slow component should represent the decay of silent synapses and AMPAR+ synapses respectively. By fitting the decay to the two-phase exponential decay model with constraining the percentage of fast component (Percent fast $_{\text {) }}$ ) to the percentage of silent synapses, I found that the decay of fast component was much faster as the time constant $\tau_{\text {fast }}$ was about ten folds smaller than $\tau_{\text {slow }}$ (same ten-fold difference in $\tau_{\text {fast }}$ and $\tau_{\text {slow }}$ was also observed in autaptic hippocampal synapses Chavis and Westbrook, 2001). This suggests that silent synapses have higher release probability.

\section{Possible functional importance of high release probability}

The ten-fold difference between $\tau_{\text {fast }}$ and $\tau_{\text {slow }}$ shows that silent synapses have much higher release probability than AMPAR + synapses, agreeing with the observation in an early study done by Yanagisawa et al. (2004). Why do silent synapses need such high release probability if they do not transmit at resting membrane potential? Since silent synapses are the substrates of LTP Kerchner and Nicoll, 2008, Liao et al., 1995, Poncer and Malinow, 2001, mechanisms favoring synaptic transmission at these synapses may exist. Perhaps the big difference between release probability of silent synapses and AMPAR+ synapses serves as a divider of signals for a neural circuit to shunt the signals to the silent synapses. High release probability at silent synapses would favor the activation of these synapses, therefore facilitates LTP formation at these synapses. Because there was no change in both $\tau_{\text {fast }}$ and $\tau_{\text {slow }}$ at P13 and P30, the difference between release probability of silent synapses and AMPAR+ synapses was maintained throughout development. 
This may indicate that maintenance of the difference in release probability could be essential for proper functioning of a neural circuit.

\section{Release probability in the calculation of silent synapse fraction}

It should be noted that calculation of silent synapse fraction from failure rates is based on the assumption that silent and AMPAR + synapses have the same release probability (see Appendix page 95 equations B.1 and B.2). If release probability ( $\mathrm{Pr}$ in the equations) of silent synapses is higher than that of AMPAR + synapses, this means that the silent synapse fraction could be overestimated. However, the exponential fitting of the NMDAR-EPSC deay with the silent synapse fraction calculated from equation B.4 yields high R-squared value, indicating that the actually silent synapse fraction is close the one estimated with equal release probability. This could probably be due to the involvement of logarithms in the calculation which would reduce the error due to a higher Pr. Nevertheless, further study is needed to validate the calculation of silent synapse fraction.

\subsubsection{Decrease in release probability during synapse maturation}

$\tau_{\text {slow }}$ did not change during development, meaning that the release probability of AMPAR + synapses did not change. This is consistent with the unchanged AMPARPPR during development. Besides, $\tau_{\text {fast }}$ did not change either, suggesting that the release probability of silent synapses was unchanged. By fitting the decay with constraining $\tau_{\text {fast }}$ and $\tau_{\text {slow }}$ of each individual recording to the values obtained in

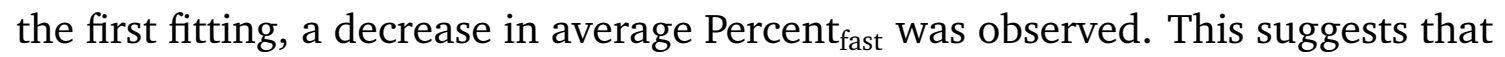
the slower decay at P30 was not due to a decrease in the release probability of synapses, but a lower percentage of silent synapses possessing possibly higher release probability. This observation agrees with the decrease in silent synapse fraction from P13 to P30. Considering these observations together, the overall release probability is likely decreased during silent synapse maturation when silent synapses are converted to AMPAR + synapses. Absence of a change or even an increase in overall release probability in previous studies with LTP activation Kullmann et al., 1996, Manabe and Nicoll, 1994) suggests that this decrease could be experience-dependent. In vitro activation of silent synapses by LTP may not be enough to induce this decrease. 
Since the maturation of silent synapses lacking AMPARs is believed to occur postsynaptically (i.e. by insertion of AMPARs to the postsynaptic sites) (Liao et al. 1995, 2001, Malinow and Malenka, 2002, simultaneous change in presynaptic release during development suggests a possible crosstalk between the two sides. A possible candidate responsible for the crosstalk would be integrin, a family of cell-adhesion molecules (CAMs) capable of mediating intercellular interaction. Chavis and Westbrook 2001 showed in autaptic hippocampal synapses that the decrease in release probability can be prevented by blocking $\beta 3$ integrin subunit. They also showed that integrin-dependent decrease in release probability may associate with the NR2B-to-NR2A subunit switch of NMDAR receptors during development. Although they did not attribute the synapses with high release probability to silent synapses, these developmental changes coincide with silent synapse maturation. Therefore, it is likely that a decrease in release probability occurs when silent synapses mature into AMPAR + synapses.

\subsection{Unlocking AMPAR transmission by TCM}

Silent synapses are believed to lack AMPARs and therefore are not transmitting at resting potential. In this study, I found that synaptic transmission was absent even at sites of transmission (synapses or compartments of synapses) where AMPARs were present. These synaptic transmission sites were uncovered in mEPSC recording as an increase in mEPSC frequency by the AMPAR desensitization blocker trichlormethiazide (TCM). These sites are "idle" in the sense that they are nontransmitting yet contain locked postsynaptic AMPARs which could be unlocked by TCM. For easier discussion, I would like to call these synaptic transmission sites "idle sites" to distinguish them from AMPAR + synapses as a general term to describe active AMPAR containing synapses, or silent synapses which lack AMPARs. In this section, I will first discuss the evidence supporting the existence of idle sites, then the reasons why they do not belong to silent synapses, where they could be situated at, and lastly whether they are silenced pre- or postsynaptically.

\subsubsection{TCM activated AMPAR-containing idle sites}

I observed a large increase in AMPAR-mEPSC frequency (more than three folds) after adding TCM at P13 and P30 (figure 3.7). Since TCM may have both presynaptic and postsynaptic effects, increase in mEPSC frequency could be due to an increase in the number of synaptic transmission sites (sites where synaptic transmission 
occurs) or release probability of AMPAR + synapses. However, because there was no change in MK-801 blocking rate of NMDARs at P30 after adding TCM (figure 3.8 , the possibility of change in release probability of AMPAR + synapses could be ruled out. Therefore, the increase in MEPSC frequency was most likely due to the recruitment of additional synaptic transmission sites which contain AMPARs, i.e. the idle sites.

\subsubsection{Idle sites are different from conventional silent synapses}

Conventionally, AMPAR-silent synapses are defined as synapses that have NMDAR transmission but not AMPAR-mediated transmission (Isaac, 2003). Although both presynaptic and postsynaptic loci of silencing have been proposed (Isaac, 2003, Kullmann, 2003, these two types of synapse silencing are not mutually exclusive. With immunogold electron-microscopic analysis, Petralia et al. 1999 showed that there are excitatory synapses containing only NMDARs but lacking AMPARs and the percentage of these synapses decreases during development. Later Montgomery et al. 2001) showed with paired recordings of pre- and postsynaptic neurons that two neurons can be connected by silent synapses which lack AMPAR transmission even when release probability is increased. These studies provides strong evidences that silent synapses lack postsynaptic AMPARs.

In this study, idle sites were shown to have AMPAR transmission by adding TCM, suggesting that they contain AMPARs and therefore do not belong to the silent synapses which lack AMPARs. Besides, EPSC recording with application of another thiazide closely related to TCM, cyclothiazide (CTZ), do not produce responses at silent synapses (Montgomery et al. 2001), suggesting that idle sites do not exist in silent synapses. In addition, if idle sites belonged to silent synapses, TCM would have a larger effects on PSD-95 KO mice which contain higher silent synapse fraction than the wild-type mice. However, the increase in mEPSC frequency by TCM was the same at P30 in wild-type and PSD-95 KO mice, suggesting that idle sites uncovered by TCM were not silent synapses. 


\section{Where are the idle sites?}

If idle sites do not belong to silent synapses, this leads us to two possibilities: (1) they are specific transmission sites found in AMPAR+ synapses; or (2) they are actually a distinct class of synapses different from AMPAR+ synapses (or "idle synapses" in the following discussion). The huge increase in mEPSC frequency during TCM application could be explained by either a large number of idle sites ( or idle synapses), a high release probability at these sites (or synapses) or a combination of these two factors.

\subsubsection{Are idle sites silenced pre- or postsynaptically?}

How would idle sites lack synaptic transmission if they contain AMPARs? Understanding how TCM unlocks these synapses can give us some hints to the answer. TCM may unlock idle sites pre- or postsynaptically, though it is primarily used as an AMPAR desensitization blocker in research with electrophysiology experiments. It is possible that AMPARs at the idle sites are desensitized by low concentration of glutamate near these sites because of the, e.g. spillover of glutamate from neighboring synapses (Kullmann et al. 1996) or restricted presynaptic release from the corresponding presynaptic terminal (Choi et al. 2000; Gasparini et al. 2000). TCM may block the desensitization and thus unsilences these idle sites.

Alternatively, TCM might unsilence idle sites presynaptically by increasing the release probability. TCM might affect presynaptic machinery through altering the intracellular potassium and calcium levels as it is known to promote excretion of potassium and calcium ions as a diuretic. However, decrease in intracellular potassium level would only affect action potential but not spontaneous transmission; decrease in intracellular calcium level would even reduce the frequency of spontaneous events which is the opposite of what I observed. Moreover, no study has shown that TCM has presynaptic actions, but it was shown for CTZ Barnes-Davies and Forsythe, 1995; Bellingham and Walmsley, 1999, Diamond and Jahr, 1995, Ishikawa and Takahashi, 2001). Although I cannot exclude the presynaptic actions of TCM on idle sites, it is unlikely to affect the presynaptic machinery of synaptic transmission at idle sites.

The absence of a change in MK-801 blocking rate of NMDARs by TCM could only exclude the possibility of TCM's presynaptic effects on the existing AMPAR+ synapses, but not on the additionally idle sites recruited during the experiment, 
because it is not known whether idle sites have evoked NMDAR transmission which MK-801 test relies on. Since idle sites presumably lack evoked transmission (the increase in evoked AMPAR-EPSC amplitude with TCM was only subtle, compared to the prominent effect of TCM on mEPSC frequency), it is possible that they may lack a machinery for evoked vesicle release. It is also possible that idle sites only contain non-transmitting AMPARs and do not have NMDARs. Such dissociation between evoked and spontaneous transmission will be discussion in section 4.4 .

Another test is required to determine whether TCM has presynaptic effects. The most direct way to test TCM's presynaptic effects would be to test the fusion propensity directly by e.g. observing the presynaptic vesicle release by fluorescence imaging. For example, one can label the presynaptic vesicles with synaptopHluorin and measure the change in fluorescence level after adding TCM. In addition, mEPSCs can be recorded simultaneously to study the connection between TCM's action on synaptic transmission and its effect on mEPSC frequency. Studying how TCM unsilences idle sites would help us to understand how these synapses are silenced.

\subsection{Dissociation of spontaneous and evoked transmission at idle sites}

According to classical view, evoked transmission is assumed to be closely related to the spontaneous transmission at the same synapse (Wasser and Kavalali, 2009). However, an increasingly number of studies have reported a dissociation between the two types of transmission Ramirez and Kavalali 2011). Different hypotheses have been proposed to explain the dissociation (Kavalali, 2014).

In this study, I show that TCM specifically increased mEPSC frequency with only a subtle effect on evoked EPSC amplitude (EPSC amplitude increased by only 1.3 folds, compared to the nearly 4 folds increase in mEPSC frequency). The small increase in EPSC amplitude was probably due to the removal of desensitization of AMPARs at AMPAR + synapses. These results suggest a dissociation of spontaneous and evoked transmission at the idle sites. They also show that evoked transmission at idle sites is probably absent and this would provide a direct evidence that spontaneous transmission and evoked transmission involve separate machineries. Although further study is needed to elucidate TCM's action on idle sites, these trans- 
mission sites provide a potential model to study the mechanism of the dissociation of the two types of transmission.

\subsubsection{Dissociation at presynapse or postsynapse?}

Dissociation of spontaneous and evoked transmission at idle sites could occur pre- or posynaptically. Presynaptically, idle sites may only have the machinery for spontaneous but not evoked transmission. Alternatively, they may have machineries for both types of transmission, but their postsynaptic AMPARs do not respond to the evoked release.

If the dissociation occurs presynaptically, it could mean that idle sites contain only the vesicle pools specific for spontaneous transmission. Vesicles for spontaneous and evoked transmission are conventionally assumed to originate from the same readily releasable pool (RRP) (Wasser and Kavalali, 2009). However, this assumption has been challenged by findings indicating that separate vesicle pools for the two transmission types may exist Chung et al. 2010, Fredj and Burrone, 2009, Koenig and Ikeda, 1999, Sara et al. 2005). Besides specific vesicle pools, idle sites may have vesicle fusion machinery specific for spontaneous transmission. Several studies have shown that spontaneous transmission and evoked transmission have different molecular requirements for the fusion machinery. Expression of alternative vesicular SNARE proteins (which are required for the formation SNARE complex for vesicle fusion) such as synaptobrevin 2 (Deák et al. 2004, Schoch et al. 2001) and VAMP7 (Hua et al. 2011) may give rise to the formation of different SNARE complexes which may regulate different types of transmission differently. Studies have also shown that same SNARE complex may have different molecular interactions with other fusion proteins such as synaptotagmin 1 or 2 , and this would also allow the differential regulation of spontaneous and evoked transmission (Liu et al. 2009, Maximov and Südhof, 2005). Finally, expression of different fusion proteins or different molecular interactions may allow modulation of fusion machinery by different pathways such as calcium signaling pathways involved in vesicle fusion Vyleta and Smith, 2011). This would result in dissociation of spontaneous and evoked transmission. Fusion machinery of idle sites may be modulated by specific pathways such that only spontaneous release is allowed.

The dissociation may also occur postsynaptically at idle sites: AMPARs at idle sites may cluster to spots on postsynaptic membrane where only spontaneous release is present on the presynaptic side. Compared to the presynaptic dissociation, there 
are far less evidences supporting the possibility of postsynaptic dissociation. By differential blocking of spontaneous and evoked transmission with use-dependent GluR2-lacking AMPARs blocker philanthotoxin, it was shown that GluR2-lacking AMPARs have distinct pools for spontaneous and evoked transmission (Sara et al. 2011). Furthermore, same observation for NMDARs was made in another earlier study with use-dependent NMDAR blocker MK-801 (Atasoy et al., 2008). These findings suggest that postsynaptic dissociation may be a general mechanism for synaptic transmission and is probably applicable to all postsynaptic receptors, including those at idle sites.

\subsubsection{Dissociation within same synapses or among different synapses?}

Dissociation of spontaneous and evoked transmission due to the difference in preor postsynaptic machinery may occur within individual synapses or among different synapses. As discussed previously, idle sites may be found in AMPAR+ synapses which have both spontaneous and evoked transmission or they could be synapses per se. Therefore, idle sites provide a potential mechanism for the dissociation occuring spatially: within individual synapses or among different synapses.

Both spatial forms of dissociation have been suggested in several studies. For dissociation within individual synapses, evidences for compartmentation of transmission machinery such as clustering of postsynaptic AMPARs provide a mechanistic basis for this possibility (MacGillavry et al. 2013). This study shows idle sites could be the possible spots which lack presynpatic machinery for spontaneous release and where AMPARs cluster to. AMPARs at idle sites may be desensitized by low glutamate concentration in synaptic cleft due to the far distance from the evoked release machinery outside idle sites (on a side note: one should be reminded that NMDARs in idle sites, if there are, may still have evoked response to presynaptic release far from them because of their much higher glutamate sensitivity Patneau and Mayer, 1990). It has been shown that binding of glutamate with a portion of AMPAR subunits of a receptor instead of all subunits can lead to the desensitization of the receptor. This provides a mechanism for AMPAR desensitization by low glutamate concentration. However, to link spatial compartmentation and dissociation of transmission types, further study is required to confirm TCM's action on AMPARs at idle sites and the clustering of these receptors in AMPAR + synapses. 
Dissociation may also occur among different synapses (Chung et al., 2010, Peled et al. 2014). It has been shown in hippocampus that either spontaneous or evoked transmission is favored in some synapses although majority of synapses display both types of transmission (Chung et al. 2010. However, dissociation among different synapses is more likely to arise from the difference in propensities for spontaneous or evoked release since difference in postsynaptic machineries between synapses has not be observed (Peled et al. 2014). Besides, while dissociation can be observed among a population of synapses (Chung et al. 2010, a single subpopulation of synapses having only spontaneous transmission has not been identified. If idle synapses exist, they would provide a convenient model to study the mechanisms for dissociation of spontaneous and evoked transmission among different synapses.

\subsection{Revising PSD-95's role in AMPAR transmission}

As a scaffold protein in postsynaptic density, PSD-95 plays an important role in AMPAR transmission (Béique and Andrade 2003). Deletion of PSD-95 causes a reduction in AMPAR function at glutamatergic synapses Beique et al., 2006, Schlueter et al. 2006), while overexpression leads to an elevated AMPAR transmission Béiqque and Andrade, 2003, Schnell et al. 2002. Besides, nanodomains within synapses have been found to be enriched in PSD-95 and AMPARs (MacGillavry et al. 2013; Nair et al., 2013). Biochemical data has shown that deletion of PSD-95 causes the reduction of AMPAR subunits GluA1 and GluA2 in PSD fraction 50\% (Bonnet et al., 2013). These results indicate that PSD-95 has important role in the regulation of AMPAR expression on the postsynaptic membrane. In addition, experience-dependent maturation of silent synapses, which has been linked to the insertion of AMPARs, requires PSD-95 during critical period of ocular dominance plasticity (Huang et al. 2015). Developmental decrease in silent synapse fraction is absent in PSD-95 KO mice. PSD-95 deletion reinstates silent synapses after critical period. Taken all evidences together, PSD-95 is believed to have critical functions in the formation of AMPAR + synapses during development. In this study, I used PSD-95 KO mice as a control which is known to have high silent synapse fraction maintained during development. In this section, I will discuss the developmental requirement of PSD-95 in the surface expression of AMPARs and synapse maturation as observed in my electrophysiological study. 


\subsubsection{PSD-95 is not a strict requirement for surface expression of AMPARs}

\section{Expression of AMPARs at postsynapse}

PSD-95 deletion only had a subtle effect on AMPAR-mEPSC amplitude during development from P11 to P30 (figure 3.1), suggesting that PSD-95 deletion only had little influence on the average AMPAR number at postsynapse. This is consistent with the implication from the findings that overexpression of PSD-95 only has effects on mEPSC frequency but not the amplitude (Béïque and Andrade, 2003), and similarly knocking down only affects the frequency but not the amplitude Krüger et al. 2013). Besides, the developmental decrease in amplitude upon eye opening observed in wild-type mice was preserved in PSD-95 KO mice. These results suggests that PSD-95 is not strictly required in the regulation of postsynaptic AMPAR number during development. Nevertheless, mEPSC frequency was reduced in PSD-95 mice by around $50 \%$ at all age groups. As discussed before, decrease in frequency likely reflect a decrease in AMPAR + synapse number instead of a change in presynaptic properties. This suggests that the 50\% decrease in AMPAR-EPSC transmission observed with PSD-95 deletion by the others (Schlueter et al. 2006) is primarily due the decrease in AMPAR+ synapse number rather than a change in number of AMPARs at individual synapses. It is possible that PSD-95 may selectively regulate the AMPAR expression at certain synapses or in certain processes, e.g. silent synapse maturation. Deletion of PSD-95 may cause a complete absence of postsynaptic AMPARs at those synapses regulated by PSD-95, without affecting the AMPAR number at other synapses. It is also possible that PSD-95 may not be required for the expression of AMPARs for spontaneous transmission, though verification of the existence of such AMPAR pool is needed. Nevertheless, effects on AMPAR expression due to PSD-95 deletion could be compensated by other MAGUKs such as PSD-93 (Elias et al., 2006, Krüger et al. 2013). In any case, the results show that PSD-95 is not strictly required for the expression of AMPARs at postsynapse.

\section{Global expression of surface AMPARs}

In this study, I tested the requirement of PSD-95 in the global expression of surface AMPARs during development by recording the current response to exogenous 
application of AMPAR agonist AMPA. With this method, I could detect AMPAR transmission from all transmitting AMPARs on the membrance surface, including synaptic and extrasynaptic AMPARs. Total AMPAR transmission, reflecting the total number of surface AMPARs, was reduced in PSD-95 KO mice at young ages but not at P30 (figure 3.10). The developmental increase in total number of surface AMPARs was not affected. Together with the results from mEPSC recordings, these results suggest that PSD-95 may be specifically required for AMPAR diffusion to the synapses or stabilization of AMPARs in the synapses instead of the surface expression of AMPARs. Biochemical studies also point to the same implication. Although AMPAR subunits GluA1 and GluA2 were reduced in PSD fraction by PSD-95 deletion (Bonnet et al. 2013), their surface expression levels (compared to the total levels) were not altered in protein crosslinking assay (Joana Duda, Göttingen; unpublished). It is possible that PSD-95 deletion causes the loss of AMPARs in some postsynapses, but these AMPARs remain on the surface outside synaptic sites. Since PSD-95 is predominantly expressed at PSD, this also indicates that the protein's role in AMPAR expression is specific to postsynapses rather than a general role in the global expression of surface AMPARs.

\section{Different roles of PSD-95 isoforms during development}

An interesting observation in $\mathrm{mEPSC}$ recording was the reduction in mEPSC frequency at P11 in PSD-95 mice (figure 3.1), despite PSD-95 expression at this age is already very low in wild-type mice (Huang et al. 2015, Sans et al. 2000). One possible explanation is that PSD-95 expression in other studies was detected with antibody which only recognizes the predominant isoform of PSD-95, PSD-95 $\alpha$, while deletion of PSD-95 in this study eliminates all isoforms. There could be an expression of another isoform, PSD-95 $\beta$ during the early stage of development. These two isoforms may play different roles in AMPAR transmission in different developmental stages: PSD-95 $\beta$ may be required for the expression of AMPARs at certain synapses during early development, but its role could be replaced by PSD-95 $\alpha$ later in development. Further study on the potential functions of PSD-95 $\beta$ in AMPAR transmission will be tested electrophysiologically with virus-mediated knockdown of PSD-95 $\beta$. 


\subsubsection{Role of PSD-95 in silent synapse maturation}

In this study, reduction in mEPSC frequency by PSD-95 deletion may be explained by the loss of AMPAR + synapses. Besides, silent synapse fraction was maintained at high level after eye opening (figure B.1). These results strengthen the argument that PSD-95 is required for the expression of AMPARs at synapses during the formation of AMPAR + synapses. However, it is questionable whether the PSD95 mediated AMPAR + synapse formation involves generation of new synapses. PSD-95 deletion did not show any effect on spine density during development (figure B.2). The unchanged spine density may indicate that PSD-95 mediates AMPAR + synapse formation primarily through silent synapse maturation. However, as discussed in Section 4.1.2, the spine density measurement might not detect the actual change in synapse density. Further study to test the change in synapse density with alternative method will be performed as mentioned before. 


\section{Bibliography}

Abbas, A. I., Yadav, P. N., Yao, W.-D., Arbuckle, M. I., Grant, S. G., Caron, M. G. and Roth, B. L. (2009). PSD-95 is Essential for Hallucinogen and Atypical Antipsychotic Drug Actions at Serotonin Receptors. The Journal of Neuroscience 29, 7124-7136.

Adesnik, H., Nicoll, R. A. and England, P. M. (2005). Photoinactivation of native AMPA receptors reveals their real-time trafficking. Neuron 48, 977-85.

Ashby, M. C. and Isaac, J. T. (2011). Maturation of a Recurrent Excitatory Neocortical Circuit by Experience-Dependent Unsilencing of Newly Formed Dendritic Spines. Neuron 70, 510-21.

Atasoy, D., Ertunc, M., Moulder, K. L., Blackwell, J., Chung, C., Su, J. and Kavalali, E. T. (2008). Spontaneous and Evoked Glutamate Release Activates Two Populations of NMDA Receptors with Limited Overlap. Journal of Neuroscience 28, 10151-66.

Balland, B., Lachamp, P., Kessler, J.-P. and Tell, F. (2008). Silent Synapses in Developing Rat Nucleus Tractus Solitarii Have AMPA Receptors. Journal of Neuroscience 28, 4624-34.

Barnes-Davies, M. and Forsythe, I. D. (1995). Pre- and postsynaptic glutamate receptors at a giant excitatory synapse in rat auditory brainstem slices. .

Béïque, J.-C. and Andrade, R. (2003). PSD-95 regulates synaptic transmission and plasticity in rat cerebral cortex. The Journal of Physiology 546, 859-67.

Beique, J.-C., Lin, D.-T., Kang, M.-G., Aizawa, H., Takamiya, K. and Huganir, R. L. (2006). Synapse-specific regulation of AMPA receptor function by PSD-95. 
Proceedings of the National Academy of Sciences 103, 19535-40.

Bellingham, M. and Walmsley, B. (1999). A Novel Presynaptic Inhibitory Mechanism Underlies Paired Pulse Depression at a Fast Central Synapse. .

Bonnet, S. A. D., Akad, D. S., Samaddar, T., Liu, Y., Huang, X., Dong, Y. and Schluter, O. M. (2013). Synaptic State-Dependent Functional Interplay between Postsynaptic Density-95 and Synapse-Associated Protein 102. Journal of Neuroscience 33, 13398-409.

Borgdorff, A. J. and Choquet, D. (2002). Regulation of AMPA receptor lateral movements. Nature 417, 649-653.

Bredt, D. and Nicoll, R. (2003). AMPA Receptor Trafficking at Excitatory Synapses. Neuron 40, 361-379.

Busetto, G., Higley, M. and Sabatini, B. (2008). Developmental presence and disappearance of postsynaptically silent synapses on dendritic spines of rat layer $2 / 3$ pyramidal neurons. .

Chater, T. E. and Goda, Y. (2014). The role of AMPA receptors in postsynaptic mechanisms of synaptic plasticity. Frontiers in Cellular Neuroscience 8.

Chavis, P. and Westbrook, G. (2001). Integrins mediate functional pre- and postsynaptic maturation at a hippocampal synapse. Nature 411, 317-321.

Cheetham, C. E. J., Hammond, M. S. L., Edwards, C. E. J. and Finnerty, G. T. (2007). Sensory Experience Alters Cortical Connectivity and Synaptic Function Site Specifically. Journal of Neuroscience 27, 3456-65.

Choi, S., Klingauf, J. and Tsien, R. W. (2000). Postfusional regulation of cleft glutamate concentration during LTP at 'silent synapses'. Nature Neuroscience 3, 330-336.

Chung, C., Barylko, B., Leitz, J., Liu, X. and Kavalali, E. T. (2010). Acute Dynamin Inhibition Dissects Synaptic Vesicle Recycling Pathways That Drive Spontaneous and Evoked Neurotransmission. Journal of Neuroscience 30, 1363-76. 
Deák, F., Schoch, S., Liu, X., Südhof, T. C. and Kavalali, E. T. (2004). Synaptobrevin is essential for fast synaptic-vesicle endocytosis. Nature Cell Biology 6, 1102-8.

del Castillo, J. and Katz, B. (1954). Quantal components of the end-plate potential. The Journal of Physiology 124, 560-573.

Deng, L. and Chen, G. (2003). Cyclothiazide potently inhibits $\gamma$-aminobutyric acid type A receptors in addition to enhancing glutamate responses. .

Derkach, V. A., Oh, M. C., Guire, E. S. and Soderling, T. R. (2007). Regulatory mechanisms of AMPA receptors in synaptic plasticity. Nat Rev Neurosci. 8, 101-113.

Desai, N., Robert, C., Nelson, S. and Turrigiano, G. (2002). Critical periods for experience-dependent synaptic scaling in visual cortex. .

Diamond, J. and Jahr, C. (1995). Asynchronous release of synaptic vesicles determines the time course of the AMPA receptor-mediated EPSC. .

Ehlers, M. D., Heine, M., Groc, L., Lee, M.-C. and Choquet, D. (2007). Diffusional Trapping of GluR1 AMPA Receptors by Input-Specific Synaptic Activity. Neuron 54, 447-460.

Elias, G. M., Funke, L., Stein, V., Grant, S. G., Bredt, D. S. and Nicoll, R. A. (2006). Synapse-Specific and Developmentally Regulated Targeting of AMPA Receptors by a Family of MAGUK Scaffolding Proteins. Neuron 52, 307-20.

Espinosa, J. S. and Stryker, M. P. (2012). Development and Plasticity of the Primary Visual Cortex. Neuron 75, 230-49.

Feldman, D. E., Nicoll, R. A. and Malenka, R. C. (1999). Synaptic Plasticity at Thalamocortical Synapses in Developing Rat Somatosensory Cortex: LTP, LTD, and Silent Synapses. J Neurobiol. 41, 92-101.

Fredj, N. B. and Burrone, J. (2009). A resting pool of vesicles is responsible for spontaneous vesicle fusion at the synapse. Nature Neuroscience 12, 751-8.

Frerking, M., Borges, S. and Wilson, M. (1997). Are Some Minis Multiquantal? J Neurophysiol. 78, 1293-304. 
Gasparini, S., Saviane, C., Voronin, L. L. and Cherubini, E. (2000). Silent synapses in the developing hippocampus: Lack of functional AMPA receptors or low probability of glutamate release? Proceedings of the National Academy of Sciences 97, 9741-6.

Gordon, J. A., Sttyker, M. P., Program, N. G. and Keck, W. M. (1996). ExperienceDependent Plasticity of Binocular Responses in the Primary Visual Cortex of the Mouse. The Journal of Neuroscience 76, 3274-3286.

Groc, L., Heine, M., Cognet, L., Brickley, K., Stephenson, F. A., Lounis, B. and Choquet, D. (2004). Differential activity-dependent regulation of the lateral mobilities of AMPA and NMDA receptors. Nature Neuroscience 7, 695 - 696.

Han, K., Cooke, S. and Xu, W. (2017). Experience-Dependent Equilibration of AMPAR-Mediated Synaptic Transmission during the Critical Period. .

Heine, M., Groc, L., Frischknecht, R., Bei que, J.-C., Lounis, B., Rumbaugh, G., Huganir, R. L., Cognet, L. and Choquet, D. (2008). Surface Mobility of Postsynaptic AMPARs Tunes Synaptic Transmission. Science 320, 201-205.

Horton, J. C. and Hocking, D. I. (1996). An Adult-Like Pattern of Ocular Dominance Columns in Striate Cortex of Newborn Monkeys prior to Visual Experience. The Journal of Neuroscience 76, 1791-1807.

Hoy, J. L. and Niell, C. M. (2015). Layer-Specific Refinement of Visual Cortex Function after Eye Opening in the Awake Mouse. Journal of Neuroscience 35, 3370-83.

Hua, Z., Leal-Ortiz, S., Foss, S. M., Waites, C. L., Garner, C. C., Voglmaier, S. M. and Edwards, R. H. (2011). V-SNARE composition distinguishes synaptic vesicle pools. Neuron 71, 474-87.

Huang, X., Stodieck, S. K., Goetze, B., Cui, L., Wong, M. H., Wenzel, C., Hosang, L., Dong, Y., Löwel, S. and Schlüter, O. M. (2015). Progressive maturation of silent synapses governs the duration of a critical period. Proceedings of the National Academy of Sciences 112, E3131-E3140.

Hubel, D. H. and Wiesel, T. N. (1959). RECEPTIVE FIELDS OF SINGLE NEURONES IN THE CAT'S STRIATE CORTEX. J. Physiol. 48, 574-591. 
Hubel, D. H. and Wiesel, T. N. (1977). Ferrier Lecture: Functional Architecture of Macaque Monkey Visual Cortex. Proc. R. Soc. Lond. B 198, 1-59.

Isaac, J. T. (2003). Postsynaptic silent synapses: Evidence and mechanisms. Neuropharmacology 45, 450-60.

Isaac, J. T. R., Nicoll, R. A. and Malenka, R. C. (1995). Neuron 15, 427-34.

Ishikawa, T. and Takahashi, T. (2001). Mechanisms underlying presynaptic facilitatory effect of cyclothiazide at the calyx of Held of juvenile rats. .

Joshi, I., Shokralla, S., Titis, P. and Wang, L. (2004). The Role of AMPA Receptor Gating in the Development of High-Fidelity Neurotransmission at the Calyx of Held Synapse. Journal of Neuroscience 24, 183-96.

Katz, L. and Shatz, C. (1996). Synaptic activity and the construction of cortical circuits. Science 274, 1133-1138.

Kavalali, E. T. (2014). The mechanisms and functions of spontaneous neurotransmitter release. Nature Reviews Neuroscience 16, 5-16.

Kennedy, M. J., Davison, I. G., Robinson, C. G. and Ehlers, M. D. (2010). Syntaxin-4 defines a domain for activity-dependent exocytosis in dendritic spines. Cell 141, 524-35.

Kerchner, G. A. and Nicoll, R. A. (2008). Silent synapses and the emergence of a postsynaptic mechanism for LTP. Nature Reviews Neuroscience 9, 813-25.

Koenig, J. H. and Ikeda, K. (1999). Contribution of Active Zone Subpopulation of Vesicles to Evoked and Spontaneous Release. J Neurophysiol. 81, 1495-505.

Krüger, J. M., Favaro, P. D., Liu, M., Kitlińska, A., Huang, X., Raabe, M., Akad, D. S., Liu, Y., Urlaub, H., Dong, Y., Xu, W. and Schlüter, O. M. (2013). Differential Roles of Postsynaptic Density-93 Isoforms in Regulating Synaptic Transmission. .

Kullmann, D. M. (2003). Silent synapses: what are they telling us about long-term potentiation? Philosophical Transactions of the Royal Society B: Biological Sciences 358, 727-33. 
Kullmann, D. M., Erdemli, G. and Asztély, F. (1996). LTP of AMPA and NMDA receptor-mediated signals: Evidence for presynaptic expression and extrasynaptic glutamate spill-over. Neuron 17, 461-74.

Liao, D., Hessler, N. A. and Malinow, R. (1995). Activation of postsynaptically silent synapses during pairing-induced LTP in CA1 region of hippocampal slice. Nature $375,400-4$.

Liao, D., Scannevin, R. H. and Huganir, R. (2001). Activation of Silent Synapses by Rapid Activity-Dependent Synaptic Recruitment of AMPA Receptors. J Neurosci. $21,6008-17$.

Liu, H., Dean, C., Arthur, C. P., Dong, M. and Chapman, E. R. (2009). Autapses and Networks of Hippocampal Neurons Exhibit Distinct Synaptic Transmission Phenotypes in the Absence of Synaptotagmin I. Journal of Neuroscience 29, 7395-403.

MacGillavry, H. D., Song, Y., Raghavachari, S. and Blanpied, T. A. (2013). Nanoscale scaffolding domains within the postsynaptic density concentrate synaptic ampa receptors. Neuron 78, 615-22.

Makino, H. and Malinow, R. (2009). AMPA Receptor Incorporation into Synapses during LTP: The Role of Lateral Movement and Exocytosis. Neuron 64, 381-90.

Malinow, R. and Malenka, R. C. (2002). AMPA receptor trafficking and synaptic plasticity. Annual Review of Neuroscience 25, 103-26.

Manabe, T. and Nicoll, R. (1994). Long-term potentiation: evidence against an increase in transmitter release probability in the CA1 region of the hippocampus. Science 265, 1888-1892.

Maximov, A. and Südhof, T. C. (2005). Autonomous function of synaptotagmin 1 in triggering synchronous release independent of asynchronous release. Neuron 48, 547-54.

Mennerick, S. and Zorumski, C. (1995). Presynaptic influence on the time course of fast excitatory synaptic currents in cultured hippocampal cells. . 
Montgomery, J. M., Pavlidis, P. and Madison, D. V. (2001). Pair recordings reveal all-silent synaptic connections and the postsynaptic expression of long-term potentiation. Neuron 29,691-701.

Nair, D., Hosy, E., Petersen, J. D., Constals, A., Giannone, G., Choquet, D. and Sibarita, J.-B. (2013). Super-Resolution Imaging Reveals That AMPA Receptors Inside Synapses Are Dynamically Organized in Nanodomains Regulated by PSD95. Journal of Neuroscience 33, 13204-24.

Nowak, L., Bregestovski, P., Ascher, P., Herbet, A. and Prochiantz, A. (1984). Magnesium gates glutamate-activated channels in mouse central neurones. Nature 307, 462-465.

O’Brien, R. J., Kamboj, S., Ehlers, M. D., Rosen, K. R., Fischbach, G. D. and Huganir, R. L. (1998). Activity-Dependent Modulation of Synaptic AMPA Receptor Accumulation. .

Patneau, D. K. and Mayer, M. L. (1990). Structure-Activity Relationships for Amino Acid Transmitter Candidates Acting at IV-MethybAspartate and Quisqualate Receptors. The Journal of Neuroscience 1, 2385-2399.

Patterson, M. A., Szatmari, E. M. and Yasuda, R. (2010). AMPA receptors are exocytosed in stimulated spines and adjacent dendrites in a Ras-ERK-dependent manner during long-term potentiation. Proceedings of the National Academy of Sciences 107, 15951-6.

Peled, E. S., Newman, Z. L. and Isacoff, E. Y. (2014). Evoked and spontaneous transmission favored by distinct sets of synapses. Current Biology 24, 484-93.

Petralia, R., Esteban, J., Wang, Y., Partridge, J., Zhao, H., Wenthold, R. and Malinow, R. (1999). Selective acquisition of AMPA receptors over postnatal development suggests a molecular basis for silent synapses. Nature Neuroscience 2, 31-36.

Poncer, J. C. and Malinow, R. (2001). Postsynaptic conversion of silent synapses during LTP affects synaptic gain and transmission dynamics. Nat Neurosci. 4, 989-96.

Ramirez, D. M. O. and Kavalali, E. T. (2011). Differential regulation of spontaneous and evoked neurotransmitter release at central synapses. Current Opinion in 
Neurobiology 21, 275-82.

Riccomagno, M. M. and Kolodkin, A. L. (2015). Sculpting Neural Circuits by Axon and Dendrite Pruning. Annual Review of Cell and Developmental Biology 31, 779-805.

Rosenmund, C., Clements, J. D. and Westbrook, G. L. (1993). Nonuniform Probability of Glutamate Release at a Hippocampal Synapse. Science 262, 754-757.

Sans, N., Petralia, R. S., Wang, Y.-X., Blahos Ii, J., Hell, J. W. and Wenthold, R. J. (2000). A Developmental Change in NMDA Receptor-Associated Proteins at Hippocampal Synapses. J Neurosci. 20, 1260-71.

Sara, Y., Bal, M., Adachi, M., Monteggia, L. M. and Kavalali, E. T. (2011). UseDependent AMPA Receptor Block Reveals Segregation of Spontaneous and Evoked Glutamatergic Neurotransmission. Journal of Neuroscience 31, 5378-82.

Sara, Y., Virmani, T., Deák, F., Liu, X. and Kavalali, E. T. (2005). An isolated pool of vesicles recycles at rest and drives spontaneous neurotransmission. Neuron 45, 563-73.

Schlueter, O. M., Xu, W. and Malenka, R. C. (2006). Alternative N-Terminal Domains of PSD-95 and SAP97 Govern Activity-Dependent Regulation of Synaptic AMPA Receptor Function. Neuron 51, 99-111.

Schnell, E., Sizemore, M., Karimzadegan, S., Chen, L., Bredt, D. S. and Nicoll, R. A. (2002). Direct interactions between PSD-95 and stargazin control synaptic AMPA receptor number. Proceedings of the National Academy of Sciences 99, 13902-7.

Schoch, S., Deák, F., Königstorfer, A., Mozhayeva, M., Sara, Y., Südhof, T. and Kavalali, E. (2001). SNARE function analyzed in synaptobrevin/VAMP knockout mice. Science 294, 1117-1122.

Smith, S. L. and Trachtenberg, J. T. (2007). Experience-dependent binocular competition in the visual cortex begins at eye opening. Nature Neuroscience 10, 370-5. 
Sur, M., Nagakura, I., Chen, N. and Sugihara, H. (2013). Mechanisms of plasticity in the developing and adult visual cortex. Progress in Brain Research 207, 243-54.

Takahashi, T., Svoboda, K. and Malinow, R. (2003). Experience Strengthening Transmission by Driving AMPA Receptors into Synapses. Science 299, 15851588.

Turrigiano, G. (1999). Homeostatic plasticity in neuronal networks: the more things change, the more they stay the same. .

Turrigiano, G. (2008). The Self-Tuning Neuron: Synaptic Scaling of Excitatory Synapses. .

Turrigiano, G., Leslie, K., Desai, N., Rutherford, L. and Nelson, S. (1998). Activitydependent scaling of quantal amplitude in neocortical neurons. .

Vyleta, N. P. and Smith, S. M. (2011). Spontaneous Glutamate Release Is Independent of Calcium Influx and Tonically Activated by the Calcium-Sensing Receptor. Journal of Neuroscience 31, 4593-606.

Wang, B. S., Sarnaik, R. and Cang, J. (2010). Critical Period Plasticity Matches Binocular Orientation Preference in the Visual Cortex. Neuron 65, 246-56.

Wasling, P., Strandberg, J. and Hanse, E. (2012). AMPA Receptor Activation Causes Silencing of AMPA Receptor-Mediated Synaptic Transmission in the Developing Hippocampus. PLOS ONE 7, 1-8.

Wasser, C. R. and Kavalali, E. T. (2009). Leaky synapses: Regulation of spontaneous neurotransmission in central synapses. Neuroscience 158, 177-88.

Yamada, K. and Tang, C. (1993). Benzothiadiazides inhibit rapid glutamate receptor desensitization and enhance glutamatergic synaptic currents. .

Yanagisawa, T., Tsumoto, T. and Kimura, F. (2004). Transiently higher release probability during critical period at thalamocortical synapses in the mouse barrel cortex: Relevance to differential short-term plasticity of AMPA and NMDA EPSCs and possible involvement of silent synapses. European Journal of Neuroscience 20, 3006-3018. 
Yang, Y., Wang, X.-B., Frerking, M. and Zhou, Q. (2008). Delivery of AMPA receptors to perisynaptic sites precedes the full expression of long-term potentiation. PNAS 105, 11388-11393.

Yao, W. D., Gainetdinov, R. R., Arbuckle, M. I., Sotnikova, T. D., Cyr, M., Beaulieu, J. M., Torres, G. E., Grant, S. G. N. and Caron, M. G. (2004). Identification of PSD-95 as a Regulator of Dopamine-Mediated Synaptic and Behavioral Plasticity. Neuron 41, 625-638. 


\section{Appendix}

\begin{tabular}{l|l|}
$\mathrm{A}$ & Statistics \\
$\mathrm{B}$ & $\mathbf{8 7}$ \\
\hline
\end{tabular} 



\section{Statistics}

Table A.1. Result summary 4 significant figures are shown. N: cell number; WT: Wild-type; KO: PSD-95 Knock-out. Age: postnatal day ( \pm 1 day). ${ }^{* 50}$ or $100 \mathrm{~ms}$ stimulus interval.

\begin{tabular}{|c|c|c|c|c|c|}
\hline Figure & Measurement & Group & Mean & S.E.M. & $\mathbf{N} / \mathbf{n}$ \\
\hline \multirow[t]{16}{*}{3.1} & \multirow[t]{8}{*}{ mEPSC frequency $(\mathrm{Hz})$} & P11 WT & 3.199 & 0.4622 & 15 \\
\hline & & P13 WT & 6.043 & 1.051 & 10 \\
\hline & & P20 WT & 5.542 & 0.7270 & 11 \\
\hline & & P30 WT & 5.700 & 0.6510 & 7 \\
\hline & & P11 KO & 1.170 & 0.1585 & 15 \\
\hline & & P13 KO & 2.810 & 0.4651 & 10 \\
\hline & & P20 KO & 3.021 & 0.4233 & 15 \\
\hline & & P30 KO & 2.994 & 0.2902 & 35 \\
\hline & \multirow[t]{8}{*}{ mEPSC amplitude (-pA) } & P11 WT & 18.50 & 0.6348 & 15 \\
\hline & & P13 WT & 15.22 & 0.3779 & 20 \\
\hline & & P20 WT & 15.75 & 0.5305 & 11 \\
\hline & & P30 WT & 15.24 & 0.6257 & 7 \\
\hline & & P11 KO & 19.42 & 1.075 & 15 \\
\hline & & P13 KO & 13.49 & 0.4231 & 10 \\
\hline & & P20 KO & 13.97 & 0.2542 & 15 \\
\hline & & P30 KO & 13.20 & 0.1990 & 35 \\
\hline \multirow[t]{3}{*}{3.2} & \multirow[t]{3}{*}{ AMPAR/NMDAR ratio } & P11 WT & 0.9719 & 0.05319 & 12 \\
\hline & & P13 WT & 1.421 & 0.08561 & 18 \\
\hline & & P30 WT & 1.993 & 0.1649 & 15 \\
\hline \multirow[t]{6}{*}{3.3} & \multirow[t]{3}{*}{ AMPAR-PPR, 50 ms* } & P11 WT & 1.223 & 0.1022 & 19 \\
\hline & & P13 WT & 1.118 & 0.06301 & 14 \\
\hline & & P30 WT & 1.044 & 0.08125 & 15 \\
\hline & \multirow[t]{3}{*}{ AMPAR-PPR, $100 \mathrm{~ms}^{*}$} & P11 WT & 1.238 & 0.1641 & 12 \\
\hline & & P13 WT & 1.012 & 0.07046 & 14 \\
\hline & & P30 WT & 0.9914 & 0.06603 & 15 \\
\hline \multirow[t]{4}{*}{3.4} & \multirow[t]{2}{*}{ AMPAR-PPR, $100 \mathrm{~ms}^{*}$} & P13 WT & 1.012 & 0.07046 & 14 \\
\hline & & P30 WT & 0.9914 & 0.06603 & 15 \\
\hline & \multirow[t]{2}{*}{ NMDAR-PPR, $100 \mathrm{~ms}^{*}$} & P13 WT & 0.4787 & 0.01641 & 14 \\
\hline & & P30 WT & 0.6662 & 0.02757 & 16 \\
\hline $3.5,3.8$ & $\tau_{\text {slow }}(\mathrm{s})$ & P13 WT & 254.2 & 29.29 & 15 \\
\hline
\end{tabular}


Table A.1. Result summary

\begin{tabular}{|c|c|c|c|c|c|}
\hline Figure & Measurement & Group & Mean & S.E.M. & $\mathbf{N}$ \\
\hline & & P30 WT & 201.0 & 21.69 & 12 \\
\hline & & P30 WT + TCM & 202.8 & 36.79 & 11 \\
\hline & $\tau_{\text {fast }}(s)$ & P13 WT & 24.49 & 4.298 & 15 \\
\hline & & P30 WT & 23.78 & 4.614 & 12 \\
\hline & & P30 WT + TCM & 24.23 & 5.551 & 11 \\
\hline & Percent $_{\text {fast }}(\%)$ & P13 WT & 50.92 & 0.5450 & 15 \\
\hline & & P30 WT & 28.08 & 0.3151 & 12 \\
\hline & & $\mathrm{P} 30 \mathrm{WT}+\mathrm{TCM}$ & 29.77 & 0.9807 & 11 \\
\hline & $\mathrm{R}^{2}$ for fitting $\tau$ & P13 WT & 0.9553 & 0.01088 & 15 \\
\hline & & P30 WT & 0.9599 & 0.01308 & 12 \\
\hline & & $\mathrm{P} 30 \mathrm{WT}+\mathrm{TCM}$ & 0.9466 & 0.01794 & 11 \\
\hline & $\mathrm{R}^{2}$ for fitting Percent ${ }_{\text {fast }}$ & P13 WT & 0.9584 & 0.009819 & 15 \\
\hline & & P30 WT & 0.9609 & 0.01276 & 12 \\
\hline & & P30 WT + TCM & 0.9531 & 0.01431 & 11 \\
\hline \multirow[t]{18}{*}{3.6} & mEPSC frequency $(\mathrm{Hz})$ & P11 WT baseline & 3.518 & 0.5019 & 7 \\
\hline & & $\mathrm{P} 11 \mathrm{WT}+\mathrm{AMPA}$ & 2.933 & 0.4870 & 7 \\
\hline & & P20 WT baseline & 6.081 & 1.010 & 7 \\
\hline & & P20 WT + AMPA & 5.097 & 0.8648 & 7 \\
\hline & & P30 WT baseline & 5.700 & 0.6510 & 7 \\
\hline & & P30 WT + AMPA & 3.796 & 0.3729 & 6 \\
\hline & mEPSC amplitude (-pA) & P11 WT baseline & 18.20 & 1.009 & 7 \\
\hline & & P11 WT + AMPA & 17.96 & 1.352 & 7 \\
\hline & & P20 WT baseline & 16.24 & 0.7810 & 7 \\
\hline & & P20 WT + AMPA & 15.53 & 0.3967 & 7 \\
\hline & & P30 WT baseline & 15.24 & 0.6257 & 7 \\
\hline & & P30 WT + AMPA & 15.40 & 0.9773 & 6 \\
\hline & Freq. (AMPA vs baseline) & P11 WT & 0.9416 & 0.1958 & 7 \\
\hline & & P20 WT & 0.8568 & 0.1155 & 7 \\
\hline & & P30 WT & 0.7050 & 0.03935 & 6 \\
\hline & Ampl. (AMPA vs baseline) & P11 WT & 0.9900 & 0.05789 & 7 \\
\hline & & P20 WT & 0.9625 & 0.02572 & 7 \\
\hline & & P30 WT & 0.9899 & 0.05173 & 6 \\
\hline \multirow[t]{4}{*}{3.7} & mEPSC frequency $(\mathrm{Hz})$ & P13 WT baseline & 5.437 & 0.8864 & 10 \\
\hline & & P13 WT + TCM & 16.70 & 2.118 & 10 \\
\hline & & P30 WT baseline & 6.774 & 1.423 & 7 \\
\hline & & P30 WT + TCM & 20.18 & 1.665 & 7 \\
\hline
\end{tabular}


Table A.1. Result summary

\begin{tabular}{|c|c|c|c|c|c|}
\hline Figure & Measurement & Group & Mean & S.E.M. & $\mathbf{N}$ \\
\hline & \multirow{6}{*}{ mEPSC amplitude (-pA) } & P30 KO baseline & 3.335 & 0.5159 & 10 \\
\hline & & P30 KO + TCM & 12.05 & 1.853 & 10 \\
\hline & & P13 WT baseline & 13.22 & 0.2943 & 10 \\
\hline & & P13 WT + TCM & 16.75 & 0.5168 & 10 \\
\hline & & P30 WT baseline & 13.56 & 0.6775 & 7 \\
\hline & & P30 WT + TCM & 16.85 & 1.000 & 7 \\
\hline & \multirow{6}{*}{ mEPSC $t_{\text {decay }}(\mathrm{ms})$} & P30 KO baseline & 12.90 & 0.2646 & 10 \\
\hline & & P30 KO + TCM & 15.32 & 0.4039 & 10 \\
\hline & & P13 WT baseline & 2.025 & 0.1099 & 10 \\
\hline & & P13 WT + TCM & 3.871 & 0.2080 & 10 \\
\hline & & P30 WT baseline & 1.955 & 0.1533 & 7 \\
\hline & & P30 WT +TCM & 3.566 & 0.1202 & 7 \\
\hline & \multirow{5}{*}{ Freq. (TCM vs baseline) } & P30 KO baseline & 1.849 & 0.03475 & 10 \\
\hline & & P30 KO + TCM & 2.967 & 0.09096 & 10 \\
\hline & & P13 WT & 3.773 & 0.7140 & 10 \\
\hline & & P30 WT & 3.516 & 0.4499 & 7 \\
\hline & & P30 KO & 3.812 & 0.3626 & 10 \\
\hline & \multirow[t]{3}{*}{ Ampl. (TCM vs baseline) } & P13 WT & 1.267 & 0.02697 & 10 \\
\hline & & P30 WT & 1.240 & 0.01966 & 7 \\
\hline & & P30 KO & 1.188 & 0.02455 & 10 \\
\hline & \multirow[t]{3}{*}{$\mathrm{t}_{\text {decay }}(\mathrm{TCM}$ vs baseline) } & P13 WT & 1.931 & 0.08838 & 10 \\
\hline & & P30 WT & 1.865 & 0.09358 & 7 \\
\hline & & P30 KO & 1.605 & 0.03995 & 10 \\
\hline 3.9 & $\begin{array}{l}\text { AMPAR-EPSC amplitude } \\
\text { (TCM vs baseline) }\end{array}$ & P30 WT baseline & 1.348 & 0.3269 & 4 \\
\hline \multirow[t]{8}{*}{3.10} & AMPA response (-pA) & P11 WT & 281.2 & 29.17 & 13 \\
\hline & & P13 WT & 420.3 & 36.26 & 9 \\
\hline & & P20 WT & 753.2 & 28.33 & 12 \\
\hline & & P30 WT & 945.2 & 68.24 & 15 \\
\hline & & P11 KO & 172.8 & 11.73 & 14 \\
\hline & & P13 KO & 301.3 & 21.01 & 7 \\
\hline & & P20 KO & 636.4 & 32.13 & 10 \\
\hline & & P30 KO & 802.0 & 38.82 & 17 \\
\hline
\end{tabular}




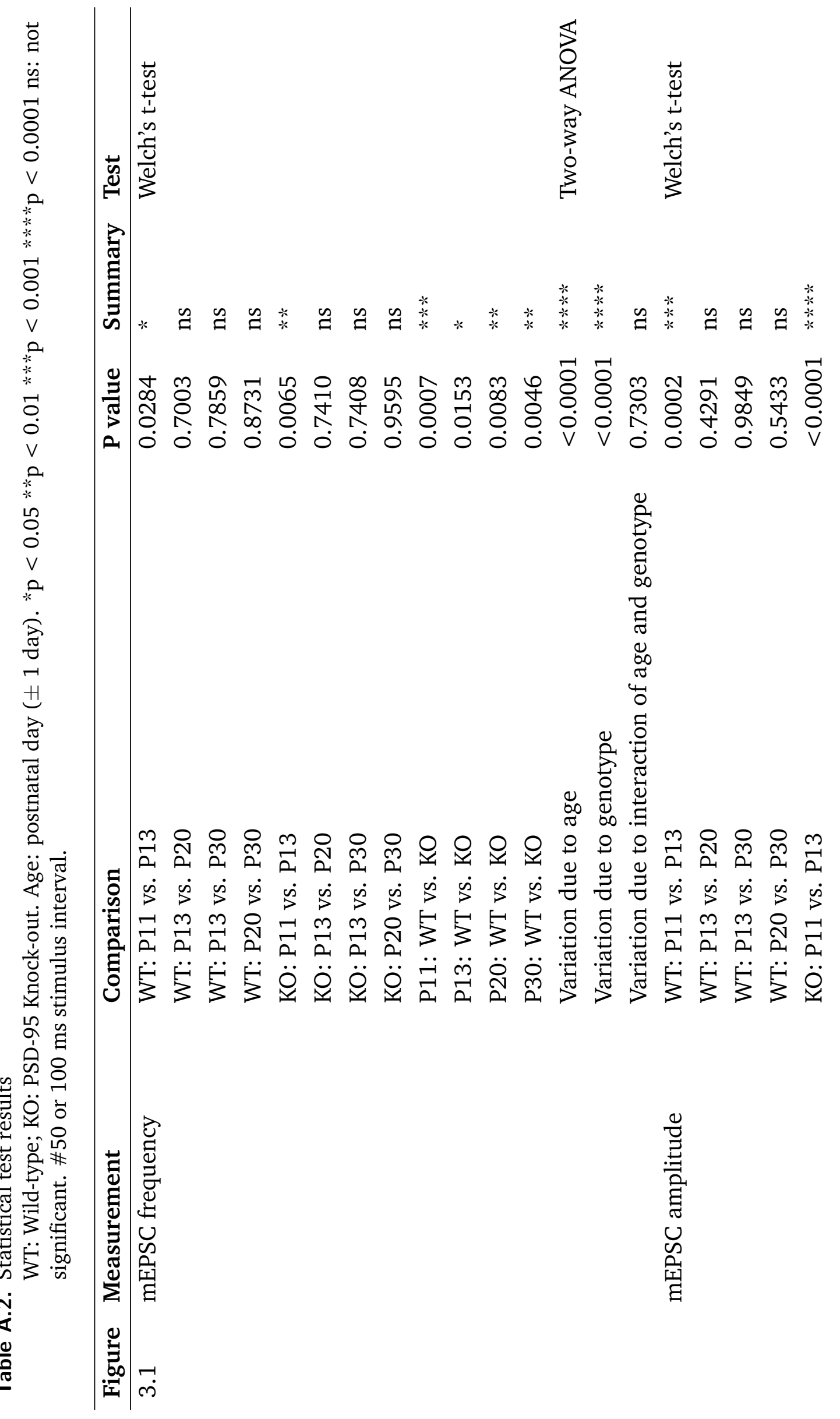




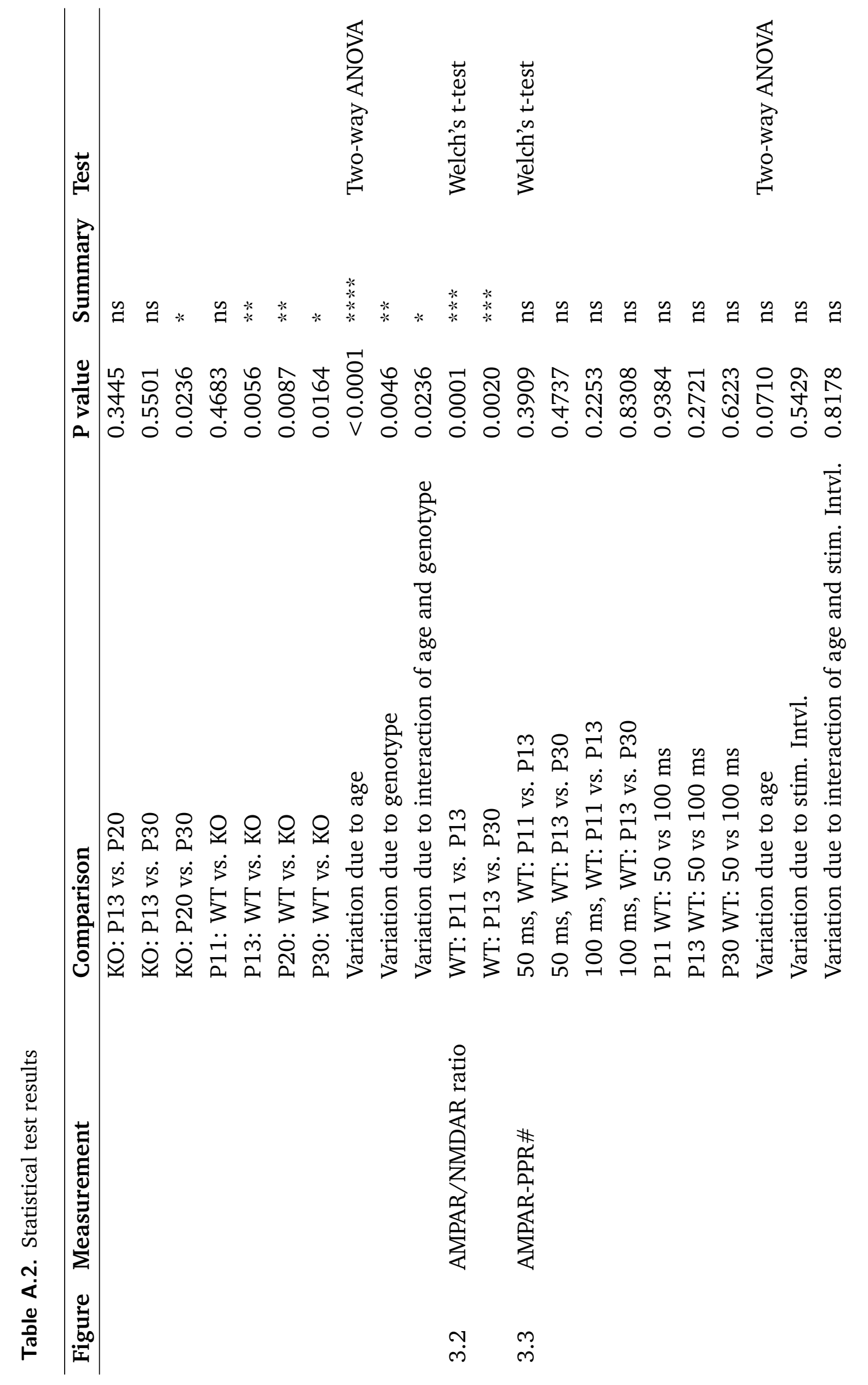




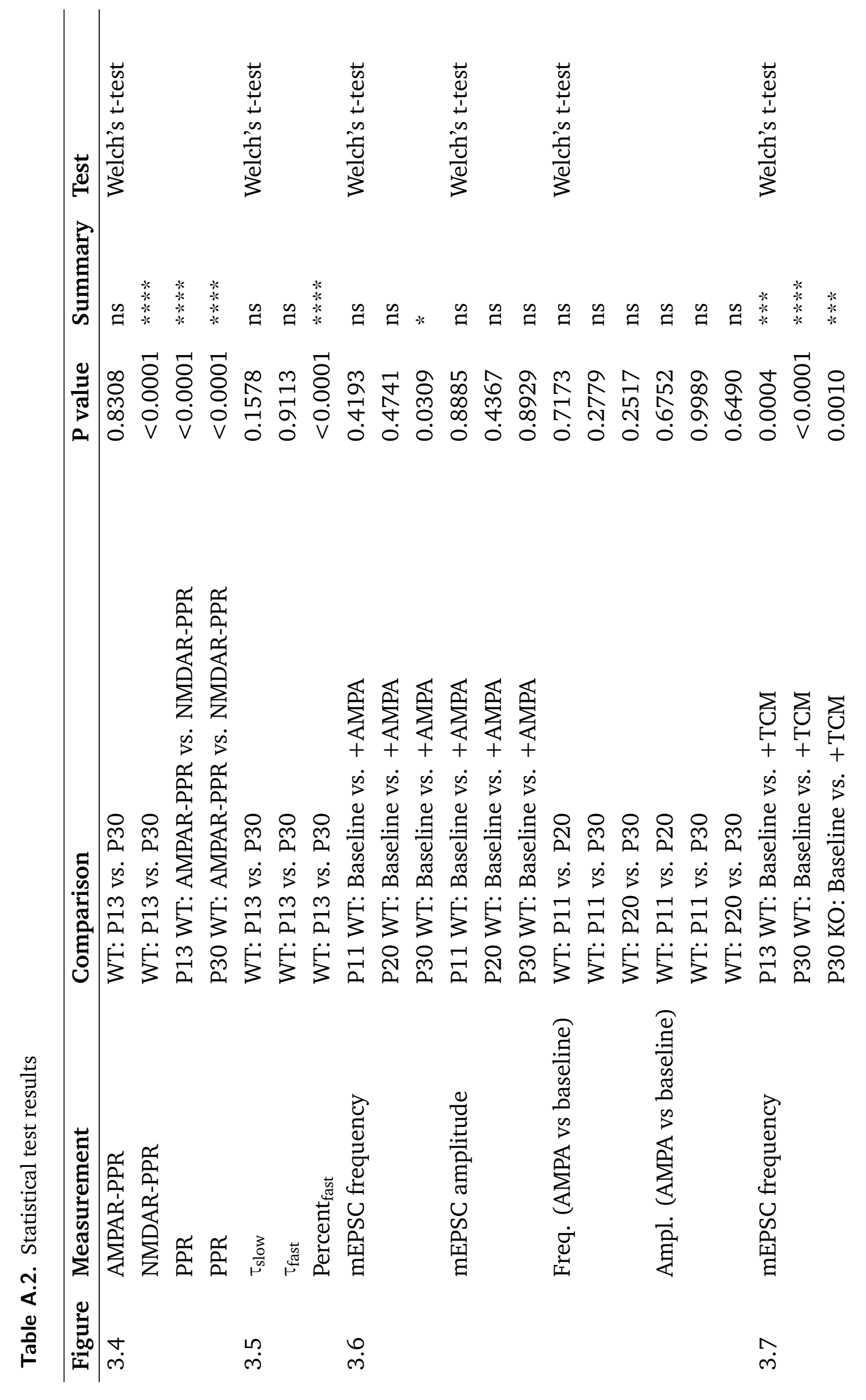




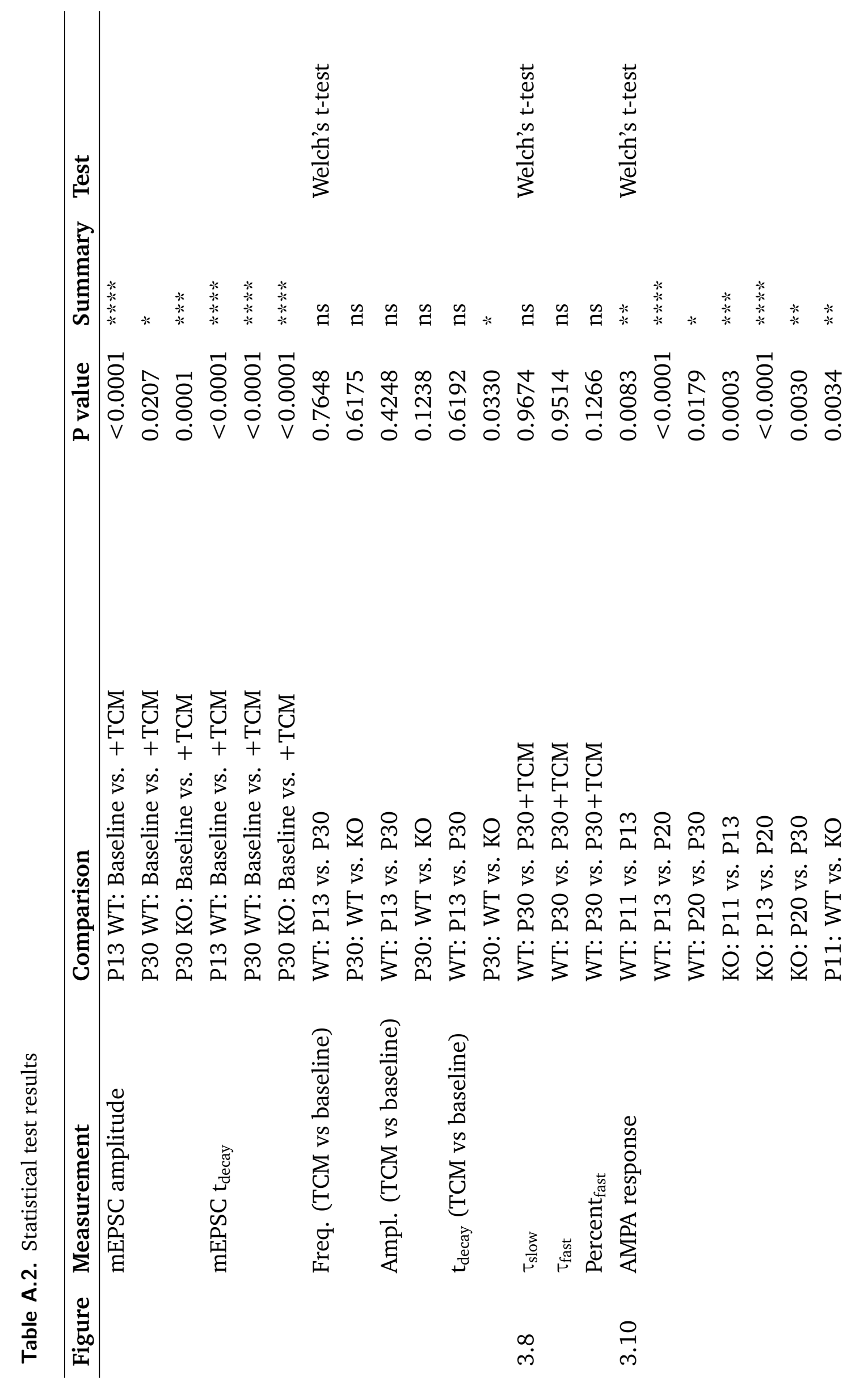




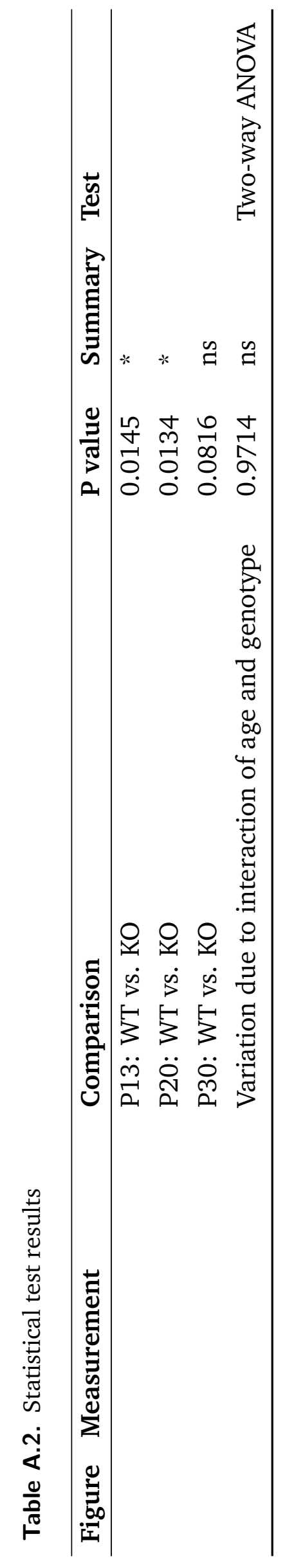




\section{Supporting figures}

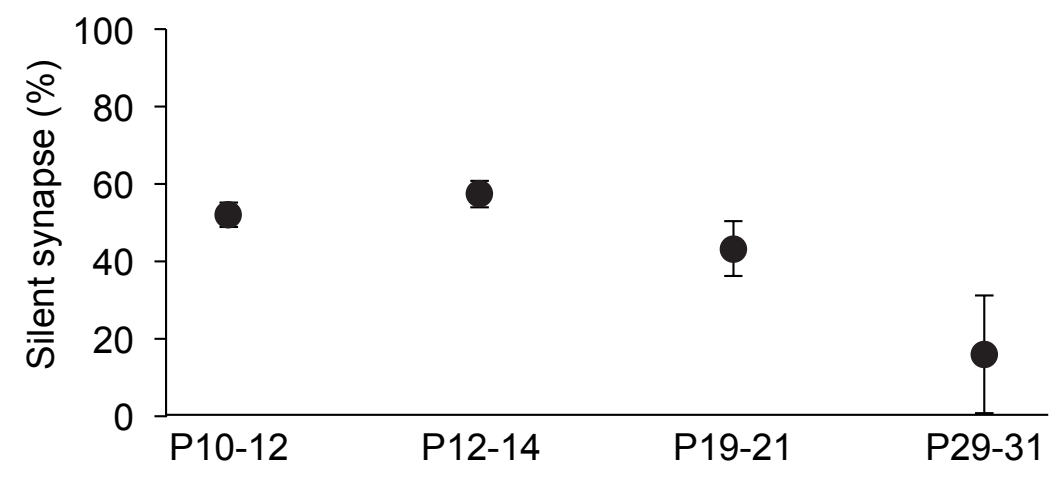

Fig. B.1. Developmental change in silent synapse fraction from P11 to P30 in L2/3 pyramidal neurons of V1 (Yuzhang Liu, Pittsburgh; Unpublished).

Silent synapse fraction was assessed with minimal stimulation as in Huang et al. (2015). Silent synapse fraction at P30 was lower than at P11 (before eye opening), consistent with the findings by Huang et al. (2015). With additional measurement at P13 (eye opening), Liu also found that silent synapse fraction was actually maintained before eye opening, and the decrease only started upon eye opening. Age: postnatal day ( \pm 1 day). Error bars: S.E.M. .

\section{Calculation of silent synapse fraction}

Silent synapse fraction in Huang et al. 2015) and figure B.1 is calculated as follow:

$$
\begin{gathered}
F_{-}=(1-P r)^{\mathrm{A}} \\
F_{+}=(1-P r)^{\mathrm{N}}
\end{gathered}
$$

where $\mathrm{F}_{-}$is the failure rate at hyperpolarized holding voltage; $\mathrm{F}_{+}$is the failure rate at depolarized holding voltage; $\mathrm{A}$ is the number of AMPAR+ synapses; $\mathrm{N}$ is the number of NMDAR + synapses (i.e. both AMPAR + and silent synapses); Pr is the release probability (assumed to be the same at AMPAR + and silent synapses). F. and $\mathrm{F}_{+}$are obtained from the experiment.

By rearranging equation B.1 and B.2, we can obtain the AMPAR+ synapse fraction and therefore the silence synapse fraction: 


$$
\frac{A}{N}=\frac{\ln F_{-}}{\ln F_{+}}
$$

Silent synapse fraction $=1-\frac{\ln F_{-}}{\ln F_{+}}$

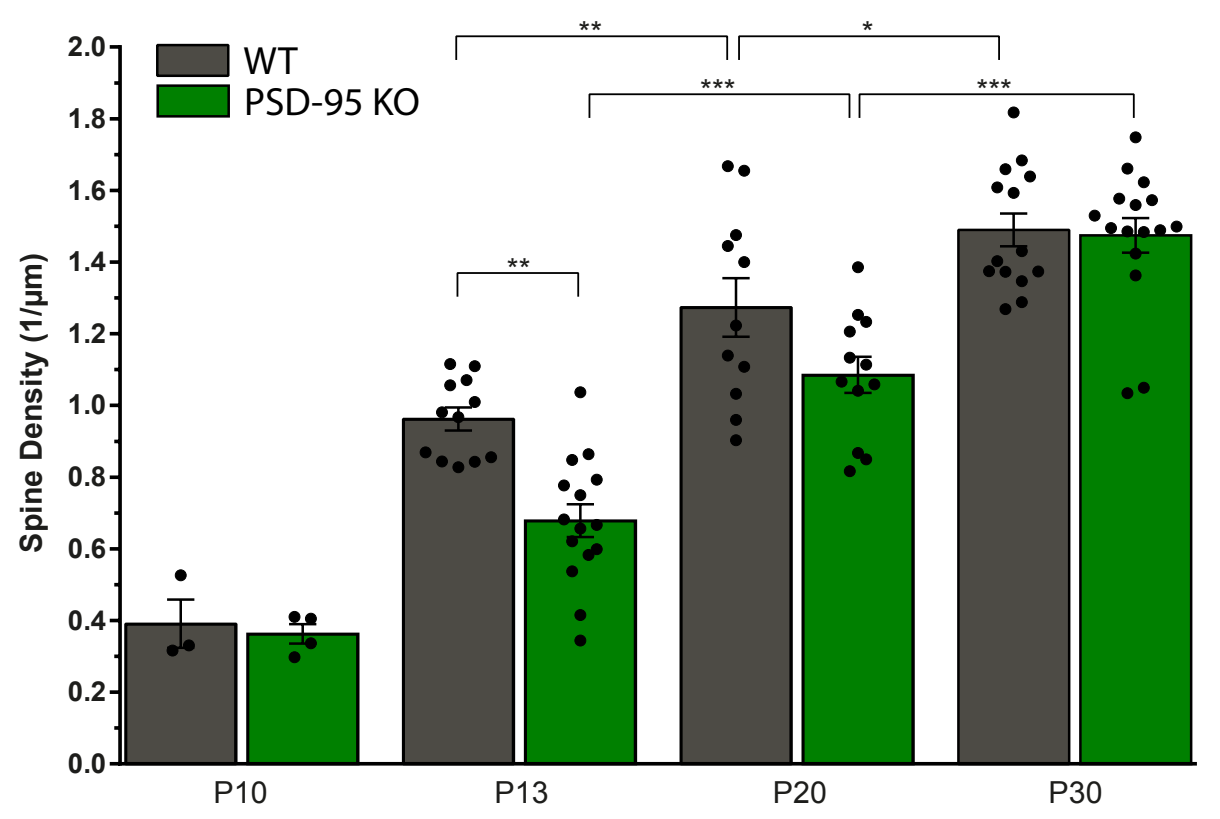

Fig. B.2. Developmental increase in spine density in L2/3 pyramidal neurons of $\mathrm{V} 1$ (Rashad Yusifov, Göttingen; unpublished).

Mouse brain was fixed with 4\% PFA and brain slices $(200 \mu \mathrm{m})$ were prepared afterwards. 5\% Lucifer Yellow was electroporated into L2/3 pyramidal neurons in V1. Secondary and tertiary basal dendrites were imaged and spines were counted. Each point: average spine density per cell. Age: postnatal day $( \pm 1$ day). Error bars: S.E.M. . *p $<0.05 * * p<0.01 * * * p<0.001$. 


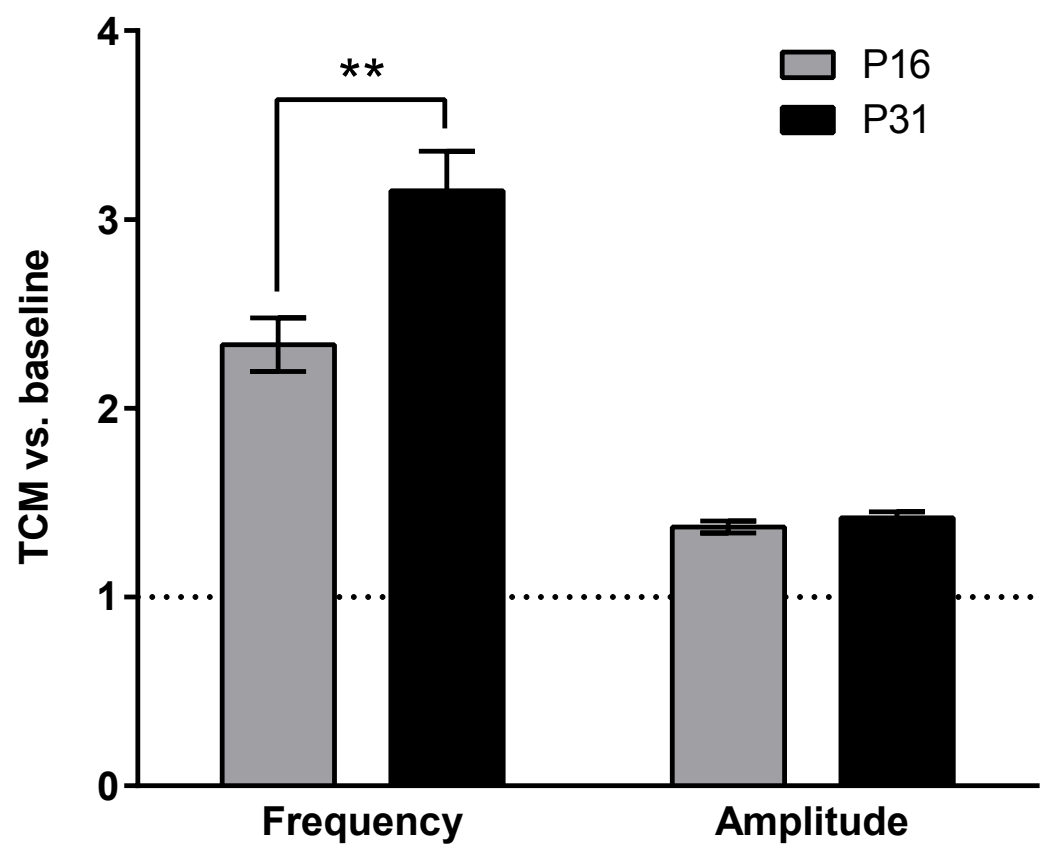

Fig. B.3. Desensitization blocker TCM had stronger effect on AMPAR-mEPSC frequency at P31 than at P16 (Yue Yang, Pittsburgh; unpublished).

Effect of TCM on AMPAR-mEPSC at P16 and P31 was assessed in the same way as in this study. With higher sample size, Yang observed a higher increase in mEPSC frequency during TCM treatment at P31 (Change: 3.152 folds; $\mathrm{N}=27$ ) than at P16 (Change: 2.336 folds; $\mathrm{N}=27$ ). Change in frequency at P16 vs. change at P31: $\mathrm{P}=0.0025$ (Welch's t-test). Error bars: S.E.M. . ${ }^{* *} \mathrm{p}<0.01$. 



\section{Acknowledgements}

First of all, I would like to thank Dr. Oliver Schlüter for his guidance through out my doctoral study. I appreciate his advice on science and beyond science. I would also like to thank my thesis committee members, Prof. Siegrid Löwel and Prof. Tobias Moser for their valuable inputs to this study.

I would also like to thank the IMPRS Neuroscience Programme, Prof. Michael Hörner and Ms. Sandra Drube for providing an excellent $\mathrm{PhD}$ programme.

I am thankful to the GGNB, the Croucher Foundation and SFB889 for their generous financially support.

I would like to European Neuroscience Institute, Göttingen for providing the infrastructure.

I would like to thank Dr. Yuzhang Liu (Pittsburgh, USA), Dr. Yue Yang (Pittsburgh, USA) and Mr. Rashad Yusifov (Göttingen) for providing their experimental data in the appendix.

Special thanks to Dr. Derya Akad who supervised me during my lab rotation and introduced me to the field of electrophysiology.

Finally, I would like to thank every member in the lab for providing a joyful and stimulating atmosphere. 



\section{Declaration}

Herewith I declare, that I prepared the Doctoral Thesis "Circuit refinement in mouse visual cortex during development" on my own and with no other sources and aids than quoted.

Göttingen, 23 $3^{\text {rd }}$ June 2017

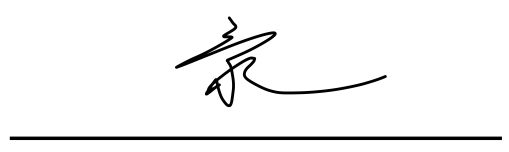

Man Ho Wong 
"We have to remember that what we observe is not nature herself, but nature exposed to our method of questioning."

Werner Heisenberg 